\title{
Wavetrain solutions of a reaction-diffusion-advection model of mussel-algae interaction
}

\author{
Matt Holzer* and Nikola Popović ${ }^{\dagger}$
}

September 20, 2016

\begin{abstract}
We consider a system of coupled partial differential equations modeling the interaction of mussels and algae in advective environments. A key parameter in the equations is the ratio of the diffusion rate of the mussel species and the advection rate of the algal concentration. When advection dominates diffusion, one observes large-amplitude solutions representing bands of mussels propagating slowly in the upstream direction. Here, we prove the existence of a family of such periodic wavetrain solutions. Our proof relies on Geometric Singular Perturbation Theory to construct these solutions as periodic orbits of the associated traveling wave equations in the large-advection-small-diffusion limit. The construction encounters a number of mathematical obstacles which necessitate a compactification of phase space, geometric desingularization to remedy a loss of normal hyperbolicity, and the application of a generalized Exchange Lemma at a loss-of-stability turning point. In particular, our analysis uncovers logarithmic (switchback) corrections to the leading-order solution.
\end{abstract}

MSC numbers: 35K57, 35C07, 35B36

Keywords: periodic wavetrain, reaction-diffusion-advection equation; geometric singular perturbation theory, geometric desingularization

\section{Introduction}

We study large-amplitude periodic wavetrains in the following system of partial differential equations (PDEs):

$$
\begin{aligned}
& u_{t}=-u_{x}+\delta(1-u-u v) \\
& v_{t}=\epsilon^{2} v_{x x}+u v-\frac{\gamma v}{1+\alpha v} .
\end{aligned}
$$

Here, the components $u$ and $v$ represent the concentrations of algae and mussels, respectively, $\epsilon$, $\alpha, \gamma$, and $\delta$ are positive parameters, while $t \in \mathbb{R}^{+}$and $x \in \mathbb{R}$ denote the temporal and spatial variables, respectively; in particular, the parameter $\epsilon$ describes the ratio of the rates of diffusion and advection in (1.1). Equation (1.1) was derived and studied numerically in [36] to model the interaction between mussels and algae in tidal flats; the resulting patterns have been found to be consistent with patterned mussel bed formation in the Wadden Sea. Of particular interest was the spontaneous formation of banded patterns comprised of large concentrations of mussels alternating

\footnotetext{
${ }^{*}$ Department of Mathematical Sciences, George Mason University, Fairfax, VA 22030, USA mholzer@gmu.edu

${ }^{\dagger}$ University of Edinburgh, School of Mathematics and Maxwell Institute for Mathematical Sciences, Edinburgh EH9 3FD, United Kingdom Nikola.Popovic@ed.ac.uk
} 
with regions of almost zero mussel concentration. These bands form perpendicular to the direction of tidal flow, and are observed over time to move slowly in the upstream direction; the dynamics of the process can be modeled as in (1.1).

A mathematical analysis of Equation (1.1) was undertaken in [39], where a homogeneous coexistence steady state was shown to be unstable due to a differential flow-induced instability. Branches of periodic solutions were also found using numerical continuation techniques, and their dependence on parameters was investigated. Analytical results were obtained in [12], where existence proofs are presented for a variety of periodic wavetrains, fronts and other traveling coherent structures that propagate with $\mathcal{O}(1)$ speeds. Recently, an alternative modeling approach was developed in [25], resulting in a Cahn-Hillard-type equation.

There exist many examples in the literature of pattern formation in reaction-diffusion-advection equations. A commonly occurring mechanism leading to the existence of these patterns is a flowinduced instability of a homogeneous steady state, see for example [27, 29, 28]. These instabilities are found in reaction-diffusion-advection equations where some of the species are subject to advection, while the others are not. Under certain conditions on the linearization about the corresponding steady state, one can deduce the existence of a critical advection rate above which the homogeneous state becomes unstable with respect to spatially heterogeneous perturbations, which is precisely the mechanism leading to the formation of patterns in Equation (1.1); cf. [39] for details. Weakly nonlinear analysis can be applied near the point of instability, whereby the bifurcation of smallamplitude periodic orbits is observed; see [29] for an example from autocatalysis and recent work in [34] for general two-component systems. We also mention the Klausmeier model [19] describing the emergence of banded vegetation patterns in arid environments as another example of a reactiondiffusion-advection equation for which a flow instability gives rise to large-amplitude wavetrains; see for example [32, 33]. Since our analysis is concerned with advection rates far above the critical rate - or, equivalently, with small diffusion rates $\epsilon$ - the details of the above mechanism do not enter directly here; nonetheless, we comment briefly on the phenomenon in Section 2.

We will consider the asymptotic limit of $\epsilon \rightarrow 0$ in Equation (1.1), which pertains to situations where advection is of significantly larger magnitude than diffusion. Numerical simulations reveal that, for small values of $\epsilon$, periodic patterns emerge which propagate in the upstream direction; see Figure 1 for an illustration. It is these periodic wavetrains that we are concerned with in the present article. We will sometimes refer to the corresponding solutions as "large-amplitude", since the $v$ component forms spikes corresponding to a surge in the concentration of mussels. Our numerical simulations further suggest that the amplitude of these spikes is not constant with respect to $\epsilon$, but that it appears to be approximately inversely proportional to $\epsilon$; cf. again Figure 1, where solutions for two different values of $\epsilon$ are compared.

We will apply Geometric Singular Perturbation Theory (GSPT) [11, 15] to prove the existence of a family of periodic wavetrains in Equation (1.1) which are parametrized by their wavespeed. Wavetrain solutions are stationary periodic solutions of (1.1) in a moving coordinate frame; therefore, they can be constructed as periodic solutions of the corresponding traveling wave ordinary differential equations (ODEs). To that end, we employ a technique developed by Trevino [35]. The existence of periodic orbits is often shown by fixing a Poincaré section and by approximating its return map, which yields the existence of a fixed point corresponding to a periodic solution. That approach is particularly fruitful when the return map is a contraction. However, the periodic solutions constructed here will be unstable as solutions of the traveling wave ODE, regardless of their stability as solutions of the original PDE. The approach in [35] was developed precisely to construct such unstable solutions: orbits in the Poincaré section are tracked in forward and backward "time" until they intersect. By augmenting the resulting manifolds with the initial values corresponding to these orbits, one can guarantee the existence of a periodic orbit if the intersection of the forward 


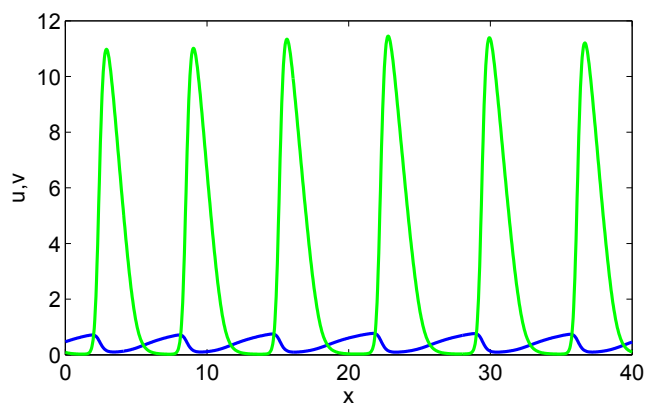

(a) $\epsilon=0.1$

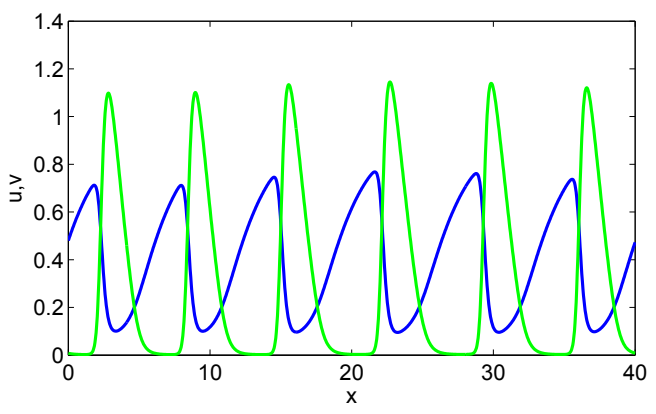

(c) $\epsilon=0.1$

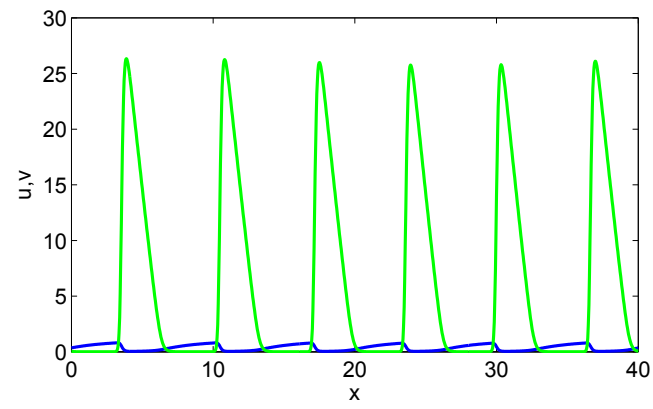

(b) $\epsilon=0.05$

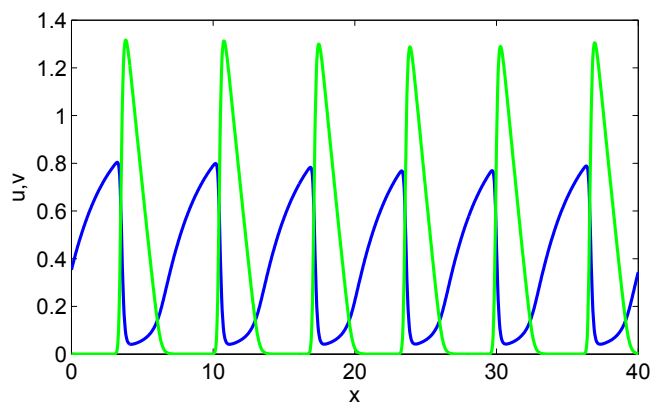

(d) $\epsilon=0.05$

Figure 1: Periodic wavetrains observed in direct numerical simulation of (1.1), with parameter values $\alpha=0.4=\delta$ and $\gamma=0.7$. Panels 1 (a) and 1(b) depict solutions, with the $u$-component in blue and the $v$-component in green. (We emphasize the difference in the scaling of $v$ in the two panels.) Panels 1(c) and 1(d) illustrate the same solutions, but with the $v$-component rescaled as $\epsilon v$. In both cases, the wavetrains are propagating to the left with $\mathcal{O}(\epsilon)$ speed. Simulations were performed using finite differences, with center differences for the diffusion operator in the $v$-component, an upwind scheme for the convection operator in the $u$-component, and periodic boundary conditions.

and backward images of the Poincaré section is transverse. The tracking itself can be performed by exploiting the smallness of the parameter $\epsilon$ : a singular orbit is identified as a concatenation of slow manifolds and fast orbits connecting those manifolds; then, nearby orbits can be tracked using a variety of techniques from GSPT.

A second challenge is presented by the large amplitude of the resulting periodic orbits. In particular, the unboundedness of the $v$-component as a function of $\epsilon$ requires a compactification of phase space. Various type of compactifications have proven useful in the literature, see for example $[40,13,22]$. Our approach is motivated by [13], where an $\epsilon$-dependent rescaling of the phase space is introduced to constrain the dynamics of a two-component ODE model for autocatalysis. (We also mention recent work in [20,23], where similar rescalings have been employed.) One consequence of the rescaling is that bounded orbits in the unscaled system are mapped to $\mathcal{O}(\epsilon)$-size regions in the rescaled variables. As is the case in [13], the resulting loss of normal hyperbolicity in the new coordinates requires us to apply geometric desingularization ("blow-up") [8, 10, 21] in order to track the evolution of orbits through phase space.

While the primary mathematical challenges encountered in the course of our analysis are related to the construction of periodic orbits in general and the application of geometric desingularization in particular, a number of other complications arise. Firstly, the requisite rescaling introduces 
$\mathcal{O}(\epsilon)$ perturbation terms whose derivatives are unbounded in certain regions of phase space, and which hence preclude the direct application of Fenichel's persistence theorems $[11,15]$. Thus, we need to utilize both the original and the rescaled variables, and then to connect the two scaling regimes. A related issue concerns the emergence of correction terms to the leading-order solution that scale not with $\epsilon$, but with $(\ln \epsilon)^{-1}$. These so-called "switchback terms" [24] arise from the matching of orbits in the two regimes. Secondly, it will turn out that our analysis requires us to track orbits in the vicinity of a slow manifold that undergoes a loss-of-stability turning point due to an intersection with another slow manifold. The resulting dynamics is described by application of generalized versions of the Exchange Lemma, which were developed in $[26,7,30]$.

The article is organized as follows. In Section 2, we review the existence and stability of homogeneous steady states for Equation (1.1). In Section 3, we introduce the corresponding traveling wave equations; we then discuss the rescaling underlying our analysis, and we state our main result. In Section 4, we analyze the resulting multiple-scale geometry in the original and the rescaled variables. In Section 5, we prepare the ground for the proof of our main result, which is then presented in Section 6. We conclude in Section 7 with comments on directions for future research. For the sake of readability, some of the longer proofs have been relegated to Appendices A and B.

\section{Existence and stability of homogeneous solutions}

In this section, we review some basic facts concerning the existence and stability of homogeneous solutions to Equation (1.1). While the information presented here is not novel [39], it is a prerequisite for the following analysis.

\subsection{Homogeneous steady states and their stability}

The homogeneous problem corresponding to (1.1) has two positive steady state solutions. The first of these is found at $(u, v)=(1,0)$ and corresponds to the state where the mussel concentration is zero, while the population of algae is in equilibrium. A second ("coexistence") steady state occurs at $(u, v)=\left(u^{*}, v^{*}\right)$, with

$$
u^{*}=\frac{\gamma-\alpha}{1-\alpha} \quad \text { and } \quad v^{*}=\frac{1-\gamma}{\gamma-\alpha} .
$$

We find two regions in parameter space for which the coexistence state is positive,

$$
\mathrm{I}=\{\gamma<1, \alpha<\gamma\} \quad \text { and } \quad \mathrm{II}=\{\gamma>1, \alpha>\gamma\}
$$

our focus will be on region I here, to which our main result will pertain. Ignoring diffusion, the stability of the homogeneous state at $\left(u^{*}, v^{*}\right)$ is determined from the eigenvalues of the Jacobian

$$
D f\left(u^{*}, v^{*}\right)=\left(\begin{array}{cc}
-\delta \frac{1-\alpha}{\gamma-\alpha} & -\delta \frac{\gamma-\alpha}{1-\alpha} \\
\frac{1-\gamma}{\gamma-\alpha} & \frac{\alpha(\gamma-\alpha)(1-\gamma)}{\gamma(1-\alpha)^{2}}
\end{array}\right),
$$

whence

$$
\begin{aligned}
\operatorname{Tr}(D f) & =-\delta \frac{1-\alpha}{\gamma-\alpha}+\frac{\alpha(\gamma-\alpha)(1-\gamma)}{\gamma(1-\alpha)^{2}} \text { and } \\
\operatorname{Det}(D f) & =\frac{\delta(1-\gamma)(\gamma+\alpha)}{\gamma(\gamma-\alpha)} .
\end{aligned}
$$

Direct calculation verifies that $\operatorname{Det}(D f)$ is always positive for $(\alpha, \gamma) \in \mathrm{I}$; the stability of $\left(u^{*}, v^{*}\right)$ is hence determined by the sign of $\operatorname{Tr}(D f)$, which is decided by the relative value of $\delta$ for $(\alpha, \gamma) \in \mathrm{I}$. 
To deduce the stability of the homogeneous state at $(1,0)$ with respect to homogeneous perturbations, we linearize again to find

$$
D f(1,0)=\left(\begin{array}{cc}
-\delta & -\delta \\
0 & 1-\gamma
\end{array}\right)
$$

Here, we observe that the mussel-free steady state is a saddle point when $\gamma<1$; in particular, it is unstable with respect to perturbations that introduce non-zero mussel concentrations into the system, but stable with respect to small perturbations of the algal concentration alone.

\subsection{Instability with respect to spatially inhomogeneous perturbations}

We now remark on the stability of the steady state $\left(u^{*}, v^{*}\right)$ with respect to spatially heterogeneous perturbations, showing that, for $\epsilon$ small, $\left(u^{*}, v^{*}\right)$ is unstable with respect to such perturbations. Scenarios such as this, wherein a stable steady state becomes unstable due to the advection of some subset of components, are known as "differential flow instabilities"; see for example [27, 29, 28].

We find it convenient to work in a reference frame moving with the speed of the algae. To do so, we let $\xi=x-t$ so that (1.1) is transformed into

$$
\begin{aligned}
u_{t} & =\delta(1-u-u v) \\
v_{t} & =\epsilon^{2} v_{\xi \xi}+v_{\xi}+u v-\frac{\gamma v}{1+\alpha v} .
\end{aligned}
$$

We then linearize Equation (2.2) about the steady state at $\left(u^{*}, v^{*}\right)$. Solutions of the linearized system exist in the form $\mathrm{e}^{\lambda t+\nu \xi}\left(u_{0}, v_{0}\right)^{T}$, where $\lambda$ and $\nu$ are given by roots of the dispersion relation

$$
d(\lambda, \nu)=\lambda^{2}-\lambda\left(\operatorname{Tr}(D f)+\epsilon^{2} \nu^{2}+\nu\right)+\operatorname{det}(D f)+A\left(\epsilon^{2} \nu^{2}+\nu\right)
$$

here, $A=-\delta\left(1+v^{*}\right)$, with $\operatorname{Tr}(D f)$ and $\operatorname{det}(D f)$ as in (2.1). The above dispersion relation is quadratic in $\lambda$. When $\epsilon=0$, there exists $\left(\omega_{\text {crit }}, k_{\text {crit }}\right) \in \mathbb{R}^{2}$ such that $d\left(i \omega_{\text {crit }}, i k_{\text {crit }}\right)=0$, and the steady state is unstable to perturbations with wavenumber $k>k_{\text {crit }}$. For small $\epsilon \neq 0$, large-wavenumber perturbations are stabilized due to diffusion, but there remains an interval of unstable wavenumbers. In other words, when the rate of advection of algae significantly exceeds the diffusion rate of mussels, the homogeneous steady state is unstable. We do not pursue a full linear stability analysis here, but instead point the reader to [39], where the differential flow instability in Equation (1.1) was studied in detail. Since the present article is concerned with the regime of arbitrarily large flow rates (relative to rates of diffusion) and large-amplitude patterns, we omit the calculation of critical flow rates and a discussion of bifurcating small-amplitude patterns, and instead refer the reader to $[27,29,28,34]$.

\section{Outline and statement of the main result}

Our aim is to construct large-amplitude periodic wavetrains in the singular limit as the diffusion parameter $\epsilon$ tends to zero in Equation (1.1), with parameter values $(\alpha, \gamma) \in$ I. In fact, a oneparameter family of wavetrains will be obtained for negative wavespeeds $\sigma$ that lie in a bounded interval; see Theorem 1 for a precise statement of our main result. We first introduce the traveling wave equations corresponding to (1.1), and we identify their multiple-scale geometry.

Wavetrains are stationary periodic solutions of (1.1) in a moving coordinate frame. We are interested in slowly propagating solutions that move upstream, i.e., to the left. Hence, we seek 
stationary solutions of Equation (1.1) in the co-moving frame defined by $\xi=x-\epsilon \sigma t$, with $\sigma<0$ :

$$
\begin{aligned}
& 0=(\epsilon \sigma-1) u_{\xi}+\delta(1-u-u v) \\
& 0=\epsilon^{2} v_{\xi \xi}+\epsilon \sigma v_{\xi}+u v-\frac{\gamma v}{1+\alpha v}
\end{aligned}
$$

Rewriting the above equations as a first-order system, using a Liénard transformation with $w=$ $\epsilon v_{\xi}+\sigma v$, we obtain

$$
\begin{aligned}
\dot{u} & =\frac{\delta}{1-\epsilon \sigma}(1-u-u v) \\
\epsilon \dot{v} & =w-\sigma v \\
\epsilon \dot{w} & =-u v+\frac{\gamma v}{1+\alpha v}
\end{aligned}
$$

For $0<\epsilon \ll 1$, Equation (3.1) is singularly perturbed, with one slow variable $u$ and two fast variables $v$ and $w$. The equations in (3.1) are often referred to as the "slow" formulation. Rescaling the independent variable to $\zeta=\frac{\xi}{\epsilon}$, we find the equivalent "fast" equations,

$$
\begin{aligned}
u^{\prime} & =\frac{\epsilon \delta}{1-\epsilon \sigma}(1-u-u v) \\
v^{\prime} & =w-\sigma v \\
w^{\prime} & =-u v+\frac{\gamma v}{1+\alpha v} .
\end{aligned}
$$

As it turns out, the original scaling of the dependent variables in Equation (3.2) is not the appropriate one for the following analysis. Indeed, the leading-order solution to (3.2) suggests that the algal concentration $u$ remains relatively constant, while the mussel population $v$ grows without bound; see Section 4.1 for a proof. In the actual solution, however, large values of $v$ will eventually lead to a decrease in $u$.

In the remainder of this section, we outline our application of GSPT to Equation (3.2), and we state our main result. Central to our approach is the identification of a singular orbit $\Gamma$ in the limit of $\epsilon=0$ in (3.2), which will consist of appropriately defined slow manifolds interspersed with fast connections between them. That orbit will provide the skeleton around which we will construct the perturbed periodic orbit for small $\epsilon>0$. As our intention here is to build intuition, all proofs will be deferred to subsequent sections. We refer the reader to Figure 2 for a sketch of the singular orbit $\Gamma$, and of the corresponding slow manifolds.

The section is organized as follows. In Section 3.1, we introduce a rescaling of the dependent variables that will capture the periodic solutions of interest. In Sections 3.2 through 3.4, we identify slow manifolds and the leading-order transitions between them, and we construct the singular orbit $\Gamma$. In Section 3.5, we state our main result. Finally, in Section 3.6, we discuss the geometric desingularization that is introduced to connect the two scaling regimes.

Remark 1. The homogeneous steady states $(1,0)$ and $\left(u^{*}, v^{*}\right)$ of $(1.1)$ correspond to equilibria for the traveling wave Equation (3.2) at $(1,0,0)$ and $\left(u^{*}, v^{*}, \sigma v^{*}\right)$, respectively. The point $\left(u^{*}, v^{*}, \sigma v^{*}\right)$ is unstable for any $\mathcal{O}(1)$ value of $\sigma$; that instability can be traced to a Hopf bifurcation which occurs for some $\sigma=\mathcal{O}(\epsilon)$, and which is eventually restabilized through another Hopf bifurcation at $\sigma=\mathcal{O}\left(\epsilon^{-1}\right)$. 


\subsection{Large-amplitude rescaling}

Motivated by [13], and with the aim of capturing large-amplitude solutions of (1.1), we define a rescaling of the dependent variables:

$$
u=U, \quad v=\frac{V}{\epsilon}, \quad \text { and } \quad w=\frac{W}{\epsilon} .
$$

In these new variables, Equation (3.1) becomes

$$
\begin{aligned}
\dot{U} & =\frac{\delta}{1-\epsilon \sigma}\left(1-U-\frac{U V}{\epsilon}\right) \\
\dot{V} & =\frac{W-\sigma V}{\epsilon} \\
\dot{W} & =-\frac{U V}{\epsilon}+\frac{\gamma V}{\alpha V+\epsilon} .
\end{aligned}
$$

The system in (3.4) is most naturally studied in terms of the independent variable $\zeta=\frac{\xi}{\epsilon}$ :

$$
\begin{aligned}
U^{\prime} & =-\delta U V+\epsilon f(U, V, \epsilon) \\
V^{\prime} & =W-\sigma V \\
W^{\prime} & =-U V+\epsilon \frac{\gamma V}{\alpha V+\epsilon},
\end{aligned}
$$

where $f(U, V, \epsilon)=\frac{\delta(1-U-\sigma U V)}{1-\epsilon \sigma}$. Note that Equation (3.5) is not expressed in slow-fast form; while such a representation could be achieved by transforming to a new variable $\widetilde{U}=U-\delta W$, we elect to work with the system in (3.5) as it is.

\subsection{Singular slow dynamics}

In this subsection, we identify slow manifolds in both the rescaled and the original coordinates; recall Equations (3.5) and (3.2), respectively.

\subsubsection{Slow-fast geometry in rescaled coordinates}

With $\epsilon=0,(3.5)$ reduces to

$$
\begin{aligned}
U^{\prime} & =-\delta U V \\
V^{\prime} & =W-\sigma V \\
W^{\prime} & =-U V .
\end{aligned}
$$

Equation (3.6) has two lines of equilibria along the $U$-axis and the $V$-axis; we denote the corresponding slow manifolds by

$$
\mathcal{M}_{U}=\{(U, V, W) \mid V=0, W=0\} \quad \text { and } \quad \mathcal{M}_{V}^{0}=\{(U, V, W) \mid U=0, W=\sigma V\} .
$$

Away from its point of intersection with $\mathcal{M}_{U}$ at the origin, the manifold $\mathcal{M}_{V}^{0}$ is normally hyperbolic; hence, GSPT implies that $\mathcal{M}_{V}^{0}$ perturbs smoothly to an invariant slow manifold $\mathcal{M}_{V}^{\epsilon}$ for $\epsilon \neq 0$ [11].

With regard to $\mathcal{M}_{U}$, the term $\frac{\epsilon V}{\alpha V+\epsilon}$ in (3.5) proves problematic, as it is not continuous at $(V, \epsilon)=(0,0)$; therefore, the perturbation $\frac{\epsilon V}{\alpha V+\epsilon}$ is no longer asymptotically smaller than the leading-order term $-U V$ when $V=o(\epsilon)$. The reduced system in (3.6) hence fails to capture the 
relevant dynamics in that regime and prevents the direct application of GSPT. We refer the reader to [1] for another example where that issue is encountered, and for a more detailed discussion. One possible resolution is a rescaling of the independent variable so that the entire vector field in Equation (3.5) is multiplied by a factor of $\alpha V+\epsilon$. The manifold $\mathcal{M}_{U}$ would then persist as a manifold of equilibria, but would not be normally hyperbolic. This inherent non-hyperbolicity of (3.5) motivates our use of geometric desingularization; however, we elect to not make the above rescaling of the vector field, as it leads to rather complicated expressions. Instead, we will only employ the rescaled coordinates $(U, V, W)$ whenever $V \geq \kappa$, where $\kappa>0$ is small, but independent of $\epsilon$.

\subsubsection{Fast-slow geometry in unscaled coordinates}

We now contrast the geometry of the rescaled system in (3.5) with that of the unscaled slow system, Equation (3.2). Here, we again find two slow manifolds,

$$
\mathcal{M}_{u}=\{(u, v, w) \mid v=0, w=0\} \quad \text { and } \quad \mathcal{M}_{v}^{0}=\left\{(u, v, w) \mid u=\frac{\gamma}{1+\alpha v}, w=\sigma v\right\} .
$$

(Since the manifold $\mathcal{M}_{u}$ is invariant irrespective of the value of $\epsilon$, there is no need to differentiate between $\epsilon=0$ and $\epsilon \neq 0$ in the notation.) The reduced dynamics on the invariant manifold $\mathcal{M}_{u}$ has a steady state at $u=1$ to which all orbits on $\mathcal{M}_{u}$ are attracted. The slow manifold $\mathcal{M}_{u}$ is normally hyperbolic away from the point $(\gamma, 0,0)$; for $u<\gamma$, the linearization transverse to $\mathcal{M}_{u}$ has eigenvalues

$$
-\frac{\sigma}{2} \pm \frac{1}{2} \sqrt{\sigma^{2}-4 u+4 \gamma}
$$

Recalling that $\sigma<0$, we observe that for $u<\gamma$, there is one stable and one unstable direction, whereas there are two unstable directions when $u>\gamma$. At $u=\gamma$, the manifold hence undergoes a loss-of-stability turning point.

Finally, we remark that the slow manifold $\mathcal{M}_{v}^{\epsilon}$ will not play a significant role in our analysis, as we are interested in $\sigma<0$, in which case $\mathcal{M}_{v}^{\epsilon}$ is repelling for $(\alpha, \gamma) \in \mathrm{I}$.

\subsection{Singular fast dynamics}

In this subsection, we describe the fast dynamics in the singular limit of $\epsilon=0$. We first consider the unscaled system, Equation (3.2), followed by the rescaled system, Equation (3.5); then, we describe how the two scaling regimes can be connected. The corresponding proofs will be presented in Section 4.

\subsubsection{Fast sub-system in unscaled coordinates}

With $\epsilon=0$ in Equation (3.2), the $u$-component is constant. We convert to projective coordinates by introducing $z=\frac{w}{v}$, whence (3.2) becomes

$$
\begin{aligned}
v^{\prime} & =v(z-\sigma) \\
z^{\prime} & =-z^{2}+\sigma z-u+\frac{\gamma}{1+\alpha v} .
\end{aligned}
$$

We focus on the case where $u<\frac{\sigma^{2}}{4}+\gamma$, in which the linearization of Equation $(3.2)$ at $(u, 0,0)$ has two real eigenvalues. With the aid of a (unbounded) trapping region that is constructed in 
Section 4.1 below, one observes that trajectories leaving $(v, w)=(0,0)$ near the corresponding strong unstable eigendirection grow without bound, approaching

$$
z=\frac{\sigma}{2}+\frac{1}{2} \sqrt{\sigma^{2}-4 u}
$$

as $\xi \rightarrow \infty$. Naturally, the resulting leading-order dynamics is only representative of the "full" system for $v=\mathcal{O}(1)$. To complete the picture, we must hence study the reduced problem in the rescaled $(U, V, W)$-coordinates.

\subsubsection{Fast sub-system in rescaled coordinates}

Consider Equation (3.6), the fast sub-system in rescaled coordinates with $\epsilon=0$. We observe that the variables $U$ and $W$ are related through $U^{\prime}=\delta W^{\prime}$ or, equivalently, through $U=\delta W+\Delta$, for some constant $\Delta$. Substituting into (3.6), we obtain the reduced planar system

$$
\begin{aligned}
V^{\prime} & =W-\sigma V \\
W^{\prime} & =-(\delta W+\Delta) V .
\end{aligned}
$$

For any $\Delta \neq 0$, the system in (3.7) has two steady states: one at the origin and one at $(V, W)=$ $\left(-\frac{\Delta}{\delta \sigma},-\frac{\Delta}{\delta}\right)$. Thus, heteroclinic orbits connecting $\mathcal{M}_{U}$ and $\mathcal{M}_{V}^{0}$ can equivalently be constructed in the planar system, Equation (3.7).

We will prove in Section 4.2 that heteroclinic connections between the two steady states of (3.7) exist for all values of $\sigma<0$ and $\Delta \leq \frac{\sigma^{2}}{4}$. As will become clear there, for $\Delta<\frac{\sigma^{2}}{4}$, these heteroclinics always depart $(V, W)=(0,0)$ along the weaker of the two unstable eigendirections; in projective coordinates, that eigendirection corresponds to

$$
Z=\frac{W}{V}=\frac{\sigma}{2}-\frac{1}{2} \sqrt{\sigma^{2}-4 \Delta}
$$

\subsubsection{Singular fast connection}

Completion of the singular orbit on the fast scale requires the identification of suitable fast connections between $\mathcal{M}_{u}$ and $\mathcal{M}_{V}^{0}$, which can be accomplished by "matching" the above two asymptotic regimes. We observe that, in unscaled coordinates, solutions that depart $\mathcal{M}_{u}$ along the strong unstable eigendirection approach infinity near

$$
u=u^{\text {out }} \quad \text { and } \quad z=\frac{\sigma}{2}+\frac{1}{2} \sqrt{\sigma^{2}-4 u^{\text {out }}}
$$

We also note the existence of connections between $\mathcal{M}_{U}$ and $\mathcal{M}_{V}^{0}$ for small $V$ when

$$
U=\Delta \quad \text { and } \quad Z=\frac{\sigma}{2}-\frac{1}{2} \sqrt{\sigma^{2}-4 \Delta} .
$$

Matching of these two conditions suggests that, to leading order, we should take $\Delta=\frac{\sigma^{2}}{4}=u^{\text {out }}$ in our construction of the singular orbit $\Gamma$.

\subsection{Description of singular orbit}

Given the information collected in Sections 3.1 through 3.3, we identify for each $\sigma<0$ a singular orbit $\Gamma$ that consists of segments of slow manifold with fast connections between them. That orbit will form the skeleton for our perturbation analysis: we will prove that there exists a periodic orbit which lies in an $o(1)$ neighborhood thereof. The sought-after singular orbit $\Gamma$ consists of several segments, as follows. 
1. Slow evolution near the manifold $\mathcal{M}_{u}$. The line of equilibria for Equation (3.2), with $u$ between zero and $u^{\text {out }}=\frac{\sigma^{2}}{4}$, constitutes the initial segment of the orbit $\Gamma$.

2. Fast connection between the manifolds $\mathcal{M}_{u}$ and $\mathcal{M}_{V}^{0}$. At $u=\frac{\sigma^{2}}{4}$, there exist fast orbits connecting $\mathcal{M}_{u}$ to arbitrarily large values of $v$; see Section 4.1. As shown in Section 4.2, the rescaled system, Equation (3.5), also admits fast connections to the slow manifold $\mathcal{M}_{V}^{0}$. A key challenge is to reconcile these scaling regimes in order to establish connections between the two families of orbits.

3. Slow evolution near the manifold $\mathcal{M}_{V}^{0}$. The next segment of $\Gamma$ is given by the portion of the slow manifold $\mathcal{M}_{V}^{0}$ that is located between $V=-\frac{\sigma}{4 \delta}$ and the non-hyperbolic steady state at the origin in (3.5).

4. Passage near the non-hyperbolic origin. To connect the singular slow manifolds $\mathcal{M}_{V}^{0}$ and $\mathcal{M}_{u}$, we utilize geometric desingularization ("blow-up"), which allows us to perform a local analysis of the flow in a neighborhood of the non-hyperbolic origin.

5. Fast return to the manifold $\mathcal{M}_{u}$. The passage past the origin in rescaled coordinates is followed by a fast transition back to $\mathcal{M}_{u}$, which completes the construction of the singular orbit $\Gamma$.

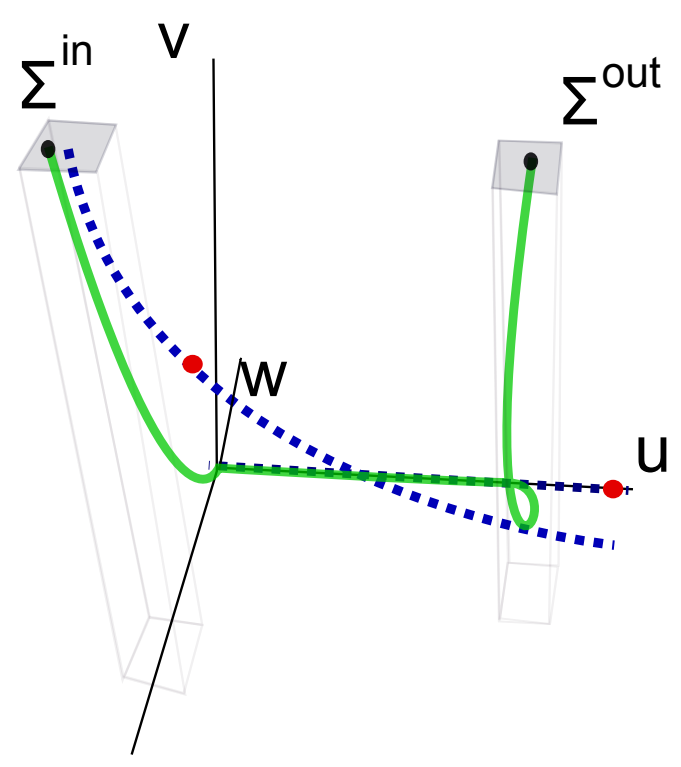

(a) Unscaled coordinates $(u, v, w)$.

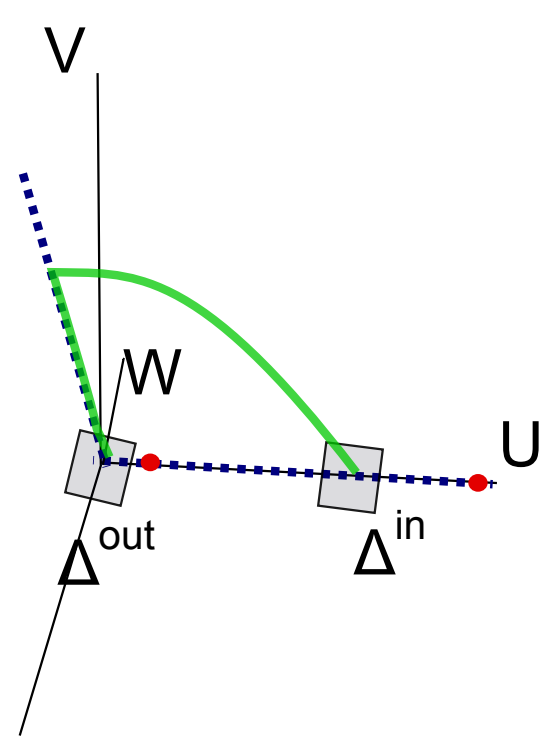

(b) Rescaled coordinates $(U, V, W)$.

Figure 2: The singular orbit $\Gamma$ in the original and the rescaled coordinates, as seen in panels 2(a) and 2(b), respectively. Blue dashed curves denote slow manifolds and red points are steady states, while the green curve represents the singular orbit. We also identify the sections $\Sigma^{\text {in }}, \Sigma^{\text {out }}, \Delta^{\text {in }}$, and $\Delta^{\text {out }}$, which are employed in the proof of our main result, Theorem 1; see Equations (4.3), (4.4), and (6.1) for precise definitions.

\subsection{Statement of main result}

Our main result concerns the existence of periodic orbits for Equation (3.5). The result pertains to orbits for which the fast connection departs $\mathcal{M}_{u}$ near its strong unstable foliation, i.e., in the 
case where $u^{\text {out }}=\frac{\sigma^{2}}{4}$.

Theorem 1. Consider $(\alpha, \gamma) \in \mathrm{I}$, and suppose $\delta>0$. Let $\sigma<0$ such that $\frac{\sigma^{2}}{4}<1$ and

$$
\int_{0}^{\frac{\sigma^{2}}{4}} \frac{-\sigma-\sqrt{\sigma^{2}-4(u-\gamma)}}{1-u} d u<0 .
$$

Then, there exists $\epsilon_{0}(\alpha, \gamma, \delta, \sigma)>0$ such that for all $\epsilon \in\left(0, \epsilon_{0}\right)$, Equation (3.5) possesses a periodic orbit $\left(U_{\text {per }}(\zeta), V_{\text {per }}(\zeta), W_{\text {per }}(\zeta)\right)$.

The periodic orbits characterized in Theorem 1 correspond to the sought-after large-amplitude wavetrains in the context of the original PDE, Equation (1.1).

Remark 2. The condition in (3.8) is required due to the presence of a loss-of-stability turning point on the manifold $\mathcal{M}_{u}$. Specifically, periodic orbits of Equation (3.5) enter a neighborhood of $\mathcal{M}_{u}$ near $u=0$; the above construction of $\Gamma$ then necessitates that these orbits exit near the (strong) unstable foliation at $u=\frac{\sigma^{2}}{4}$. If $\frac{\sigma^{2}}{4}<\gamma$, any manifold that enters a neighborhood of $\mathcal{M}_{u}$ near $u=0$ along its stable foliation is guaranteed to possess orbits that exit near $u=\frac{\sigma^{2}}{4}$. However, for $\frac{\sigma^{2}}{4}>\gamma$, the linearization transverse to $\mathcal{M}_{u}$ has two unstable eigenvalues, which implies that solutions could be forced to exit the neighborhood prematurely. The condition in (3.8) precludes that possibility; see Appendix A for a precise definition and a more in-depth discussion.

We also note that we will only be interested in $u \in\left[-c_{u}, \frac{\sigma^{2}}{4}+c_{u}\right]$ for some small constant $c_{u}>0$. For that reason, we may always assume $\sigma^{2}-4 u+4 \gamma>0$ and, hence, that the linearization transverse to $\mathcal{M}_{u}$ only has real eigenvalues.

Remark 3. We emphasize that the wavespeed $\sigma$ is negative throughout this article. Since the advective term in (1.1) breaks the $\{x \rightarrow-x\}$-symmetry of the system, upstream and downstream solutions are fundamentally distinct. In particular, none of the following analysis applies in the case where $\sigma>0$.

Remark 4. Since the proof of Theorem 1 is constructive, our analysis could be refined to yield estimates for the period and the amplitude of the resulting periodic orbit for Equation (3.5) as a function of its parameters. In particular, Lemma 6.2 below suggests the presence of logarithmic (switchback) corrections in $\epsilon$ in the corresponding asymptotic expansions.

\subsection{Geometric desingularization}

We remark on geometric desingularization, or the blow-up technique [8, 10, 21], which will be employed in our proof of Theorem 1. We have already seen that two distinct scaling regimes will be required to resolve the dynamics of Equation (3.2). Connecting the dynamics in the two regimes involves tracking orbits through regions where GSPT does not apply directly - i.e., those where either $v \rightarrow \infty$ or $V \rightarrow 0$ - which will be accomplished via geometric desingularization.

Consider Equation (3.5), with the trivial equation $\epsilon^{\prime}=0$ appended. In desingularizing ("blowing up") the slow manifold $\mathcal{M}_{u}$, each point $(U, V, W, \epsilon)$ is mapped to a sphere with coordinates $(U, r, \bar{V}, \bar{W}, \bar{\epsilon})$, where $\bar{V}^{2}+\bar{W}^{2}+\bar{\epsilon}^{2}=1$. Rather than consider the dynamics in these coordinates, it is typically convenient to introduce coordinate charts in which the "blown-up" dynamics simplifies. We utilize two charts here, with

$$
U=u_{1}, \quad V=r_{1} v_{1}, \quad W=r_{1} w_{1}, \quad \text { and } \quad \epsilon=r_{1}
$$


and

$$
U=u_{2}, \quad V=r_{2}, \quad W=r_{2} w_{2}, \quad \text { and } \quad \epsilon=r_{2} \epsilon_{2},
$$

which we denote by $K_{1}$ and $K_{2}$, respectively; one can transform between these charts via

$$
u_{2}=u_{1}, \quad w_{2}=\frac{w_{1}}{v_{1}}, \quad r_{2}=r_{1} v_{1}, \quad \text { and } \quad \epsilon_{2}=\frac{1}{v_{1}} .
$$

We note that, in chart $K_{1}$, the coordinate $r_{1}$ plays the role of $\epsilon$, while $u_{1}, v_{1}$, and $w_{1}$ are precisely the unscaled variables $u, v$, and $w$, respectively. The coordinates in chart $K_{2}$ can be interpreted as follows: $w_{2}=\frac{W}{V}$ is the usual projectivized coordinate, whereas $\epsilon_{2}=\frac{\epsilon}{V}=\frac{1}{v}$ captures the rescaled behavior of orbits near infinity.

Remark 5. As remarked above, the term $\frac{\epsilon V}{V+\alpha \epsilon}$ is no longer an asymptotically small perturbation when $V=o(\epsilon)$. While multiplying the entire vector field in Equation (3.5) with the factor $V+\alpha \epsilon$ would remove that obstacle, we opt to leave the system as it is. Rather, we insist on using the rescaled coordinates only when $V$ is $\mathcal{O}(1)$ or larger. Orbits with $V=o(1)$, on the other hand, are studied in blown-up coordinates.

For future reference, we note that, in the coordinates of charts $K_{1}$ and $K_{2}$, Equation (3.5) reads

$$
\begin{aligned}
u_{1}^{\prime} & =-\delta r_{1} u_{1} v_{1}+r_{1} f\left(u_{1}, r_{1} v_{1}, r_{1}\right) \\
v_{1}^{\prime} & =w_{1}-\sigma v_{1} \\
w_{1}^{\prime} & =-u_{1} v_{1}+\gamma \frac{v_{1}}{1+\alpha v_{1}} \\
r_{1}^{\prime} & =0
\end{aligned}
$$

and

$$
\begin{aligned}
u_{2}^{\prime} & =-\delta r_{2} u_{2}+\epsilon_{2} r_{2} f\left(u_{2}, r_{2}, r_{2} \epsilon_{2}\right) \\
r_{2}^{\prime} & =r_{2}\left(w_{2}-\sigma\right) \\
w_{2}^{\prime} & =-w_{2}\left(w_{2}-\sigma\right)+\epsilon_{2} \frac{\gamma}{\alpha+\epsilon_{2}}-u_{2} \\
\epsilon_{2}^{\prime} & =-\epsilon_{2}\left(w_{2}-\sigma\right),
\end{aligned}
$$

respectively; here, the function $f$ is defined as in Section 3.1.

Remark 6. We will require a variety of coordinate systems in the remainder of the article. In theory, our entire analysis could be performed exclusively in the coordinates of charts $K_{1}$ and $K_{2}$. However, the dependence of the corresponding vector fields on the parameter $\epsilon$ is opaque and not well suited to the application of GSPT. Hence, we will use a combination of the unscaled coordinates $(u, v, w)$, the rescaled coordinates $(U, V, W)$, as well as those of chart $K_{2}$, in our proof of Theorem 1. In particular, the latter coordinates are useful when connecting the two scaling regimes corresponding to the former two coordinate systems. In the process, we find it convenient to employ the projective coordinates $z=\frac{w}{v}$ and $Z=\frac{W}{V}$, as well as a compactification of the unscaled variables via $y=\frac{1}{v}$. Since the coordinates of chart $K_{1}$ are equivalent to the unscaled ones, we will not explicitly use them. Naturally, in our analysis, we will also introduce a variety of other coordinate systems, such as, for example, normalizing coordinates in the vicinity of invariant manifolds.

\section{Fast dynamics in the original and rescaled coordinates}

Before proceeding to the proof of Theorem 1, we study the singular fast dynamics of Equation (3.1), compiling several results that will be required in the following analysis. In the process, we substantiate our choice of $u^{\text {out }}=\frac{\sigma^{2}}{4}$ in the construction of the singular orbit $\Gamma$. 


\subsection{Fast connections between $\mathcal{M}_{u}$ and infinity}

In this subsection, we turn our attention to the fast transition from the slow manifold $\mathcal{M}_{u}$ to infinity.

Consider the traveling wave equations in the original scaling; recall Equation (3.1). For some fixed value of $\sigma$, the singular orbit $\Gamma$ then consists of a jump forward from the slow manifold $\mathcal{M}_{u}$ at $u=\frac{\sigma^{2}}{4}$ and a jump back near $u=0$. We need to describe how orbits approach infinity in the unscaled variables $(u, v, w)$. To that end, we will utilize projective coordinates: let $z=\frac{w}{v}$; then, Equation (3.1) becomes

$$
\begin{aligned}
u^{\prime} & =\frac{\epsilon \delta}{1-\epsilon \sigma}(1-u-u v) \\
v^{\prime} & =-\sigma v+z v \\
z^{\prime} & =-z^{2}+\sigma z-u+\frac{\gamma}{1+\alpha v} .
\end{aligned}
$$

To study the behavior of orbits with arbitrarily large $v$-component, we write $y=v^{-1}$, whence (4.1) is transformed to

$$
\begin{aligned}
u^{\prime} & =\frac{\epsilon \delta}{1-\epsilon \sigma}\left(1-u-\frac{u}{y}\right) \\
y^{\prime} & =\sigma y-z y \\
z^{\prime} & =-z^{2}+\sigma z-u+\frac{\gamma y}{y+\alpha} .
\end{aligned}
$$

We note that, as $v \rightarrow \infty$ or, equivalently, as $y \rightarrow 0$, the $u$-component is no longer a slow variable due to the terms $u v$ and $u y^{-1}$ in Equations (4.1) and (4.2), respectively. In a first step, we set $\epsilon=0$, and we study the dynamics of the resulting reduced equations for all $v \in \mathbb{R}$. When perturbing to $\epsilon \neq 0$, we will then restrict our attention to orbits which remain uniformly bounded in $\epsilon$. Continuation of these orbits to larger values of $v$ will be performed in the coordinates of chart $K_{2}$; recall Section 3.6.

Next, we state some basic facts concerning Equations (4.1) and (4.2). For $\epsilon=0$ in (4.1) and $u$ fixed, there exist steady states at $(v, z)=\left(0, z_{v}^{ \pm}\right)$, with

$$
z_{v}^{ \pm}=\frac{\sigma}{2} \pm \frac{1}{2} \sqrt{\sigma^{2}-4(u-\gamma)}
$$

these states are real when $u<\frac{\sigma^{2}}{4}+\gamma$. The steady states at $\left(0, z_{v}^{ \pm}\right)$correspond to the eigendirections of (4.1) at $v=0$; specifically, $z_{v}^{+}$represents the (strong) unstable eigendirection, which can be positive or negative depending on whether or not the corresponding eigenvalue is greater than $-\sigma$. Conversely, $z_{v}^{-}$corresponds to the stable or weak unstable eigendirection. The steady state at $(v, z)=\left(0, z_{v}^{+}\right)$is always a saddle point, while the one at $\left(0, z_{v}^{-}\right)$is a saddle when $u>\gamma$ and an unstable node when $u<\gamma$. Finally, when $u<\gamma$, there also exists a third steady state at $\left(\frac{\gamma-u}{\alpha u}, \sigma\right)$, corresponding to a point on the slow manifold $\mathcal{M}_{v}^{0}$.

For $\epsilon=0$ in (4.2) and $u$ fixed, there exist steady states at $(y, z)=\left(0, z_{y}^{ \pm}\right)$, with

$$
z_{y}^{ \pm}=\frac{\sigma}{2} \pm \frac{1}{2} \sqrt{\sigma^{2}-4 u}
$$

The state at $\left(0, z_{y}^{+}\right)$is stable, while the one at $\left(0, z_{y}^{-}\right)$is a saddle point when $u>0$ and an unstable node when $u<0$. When $0<u<\gamma$, there also exists a positive steady state at $(y, z)=\left(\frac{u \alpha}{\gamma-u}, \sigma\right)$, which is an unstable node. Note that, as $u$ passes through zero, that steady state collides with the state at $\left(0, z_{y}^{+}\right)$, undergoing a transcritical bifurcation. 
We now prove the existence of heteroclinic orbits connecting the steady states at $v=0$ in Equation (4.1) to those at $y=0$ in (4.2). The proof is developed in the following two subsections; see also Figure 3.

\subsubsection{Dynamics near take-off point $\left(u^{\text {out }}=\frac{\sigma^{2}}{4}\right)$}

We begin by discussing the dynamics near the take-off point $u^{\text {out }}$; to that end, we introduce the section

$$
\Sigma^{\text {out }}=\left\{(u, v, z) \mid u \in\left[\frac{\sigma^{2}}{4}-c_{u}, \frac{\sigma^{2}}{4}+c_{u}\right], v=\frac{1}{\kappa}, z \in\left[\frac{\sigma}{2}-c_{z}, \frac{\sigma}{2}+c_{z}\right]\right\}
$$

for the flow of Equation (4.1), see Figure 2. (Here, $c_{u}$ and $c_{z}$ are sufficiently small and positive constants, while $\kappa$ is defined as in Section 3.2.)

Lemma 4.1. Let $\kappa>0$ and $\sigma<0$, and consider $u^{\text {out }} \in\left[\frac{\sigma^{2}}{4}-c_{u}, \frac{\sigma^{2}}{4}+c_{u}\right]$. Then, for $\epsilon=0$ in Equation (4.1), the intersection of the unstable manifold of the steady state at $\left(0, z_{v}^{+}\right)$with the section $\Sigma^{\text {out }}$ can be represented as the graph $z=H^{\mathrm{uu}}\left(u^{\mathrm{out}}\right)$. Here, the function $H^{\mathrm{uu}}\left(u^{\mathrm{out}}\right)$ is monotonically decreasing.

Proof: We first consider the case where $u^{\text {out }}=\frac{\sigma^{2}}{4}$, which we then extend to nearby values of $u$. When $u^{\text {out }}=\frac{\sigma^{2}}{4}$, the two steady states at infinity - i.e., at $y=0$ in $(4.2)$ - undergo a saddlenode bifurcation at $(y, z)=\left(0, \frac{\sigma}{2}\right)$. We show the existence of a heteroclinic connection between $(v, z)=\left(0, \frac{\sigma}{2}+\sqrt{\gamma}\right)$ and $(y, z)=\left(0, \frac{\sigma}{2}\right)$. To that end, let $z_{\max }=\max \left\{0, \frac{\sigma}{2}+\sqrt{\gamma}\right\}$, and consider the rectangular strip $\mathcal{R}$ defined by $z \in\left[\frac{\sigma}{2}, z_{\max }\right]$ and $v \in \mathbb{R}$. It is easy to verify that the flow of (4.1) always points into $\mathcal{R}$ on the lines $\left\{z=z_{\max }\right\}$ and $\left\{z=\frac{\sigma}{2}\right\}$. Hence, $\mathcal{R}$ is a trapping region, which implies the existence of the sought-after heteroclinic connection.

Now, let $\tilde{u}=u^{\text {out }}-\frac{\sigma^{2}}{4}$. For $\tilde{u}<0$, there exist two steady states at infinity, while there are none for $\tilde{u}>0$. Nonetheless, the above argument can be extended; we also know that the unstable manifold of $(v, z)=\left(0, \frac{\sigma}{2}+\sqrt{\gamma}\right)$ is guaranteed to intersect the section $\Sigma^{\text {out }}$ for any $\tilde{u}<\frac{\gamma}{1+\alpha \kappa}$.

Let $H^{\text {uu }}\left(u^{\text {out }}\right)$ denote the $z$-value of that intersection, and note that the trapping region $\mathcal{R}$ can be improved as follows. Given some fixed value $u^{*} \in\left[\frac{\sigma^{2}}{4}-c_{u}, \frac{\sigma^{2}}{4}+c_{u}\right]$, we can calculate the vector field along the corresponding orbit, which we denote by $\left(t_{v}\left(u^{*}, v, z\right), t_{z}\left(u^{*}, v, z\right)\right)^{T}$; for $u \neq u^{*}$, the vector field reads

$$
\left(t_{v}\left(u^{*}, v, z\right), t_{z}\left(u^{*}, v, z\right)+\left(u^{*}-u\right)\right)^{T},
$$

which implies that for $u>u^{*}$, the heteroclinic orbit found for $u^{*}$ forms a boundary for the improved trapping region, as well. Finally, since the $z$-component of the steady state $(v, z)=\left(0, \frac{\sigma}{2}+\sqrt{\gamma-\tilde{u}}\right)$ decreases as $\tilde{u}$ increases, we conclude that $H^{\text {uu }}(u)$ is a decreasing function of $u$, as claimed.

Remark 7. When $u^{\text {out }}<\gamma$, Equation (4.2) has an additional steady state for $\epsilon=0$, corresponding to a point on the slow manifold $\mathcal{M}_{v}^{0}$. Since that state lies to the left of the line $\{z=\sigma\}$, it does not affect the above analysis; see, however, Lemma 4.2 below, where the additional steady state on $\mathcal{M}_{v}^{0}$ will need to be accounted for.

\subsubsection{Dynamics near touch-down point $(u=0)$}

Next, we consider the dynamics near the touch-down point at the origin in Equation (4.1); the corresponding section for the flow is defined as

$$
\Sigma^{\text {in }}=\left\{(u, v, z) \mid u \in\left[-c_{u}, c_{u}\right], v=\frac{1}{\kappa}, z \in\left[\sigma-c_{z}, \sigma+c_{z}\right]\right\}
$$




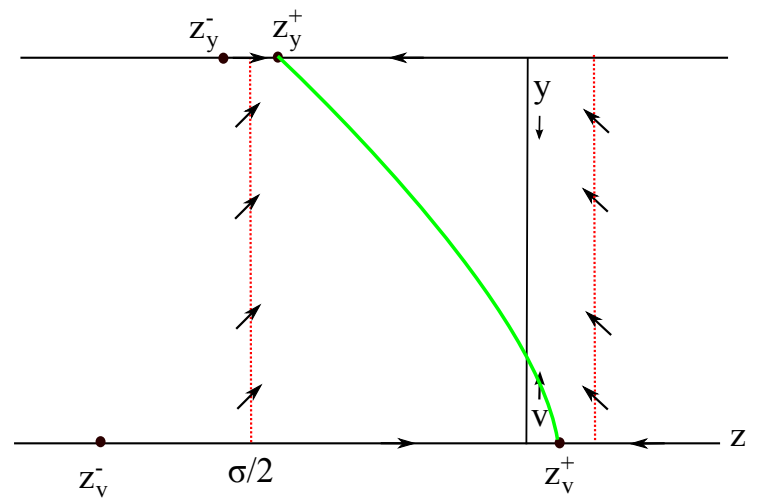

(a) Trapping region $\mathcal{R}$.

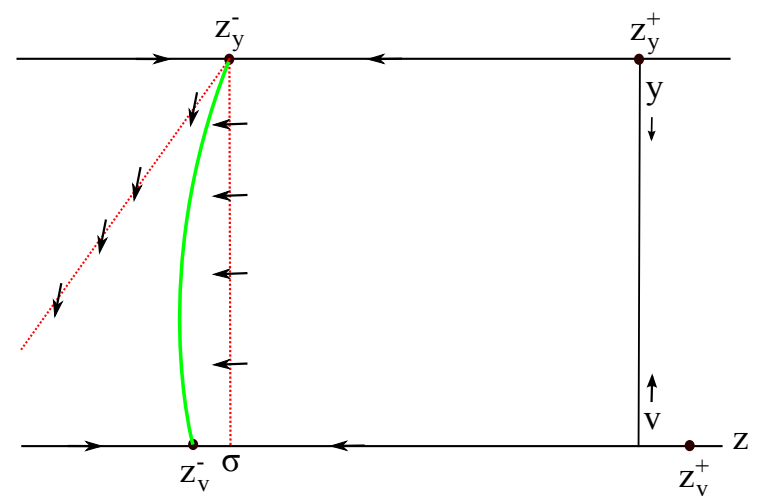

(b) Trapping region $\mathcal{S}$.

Figure 3: The compactified vector fields in Equations (4.1) and (4.2) and their trapping regions. Red dashed lines depict the boundaries of these regions, while green curves represent heteroclinic connections. In panel 3(a), we illustrate the proof of Lemma 4.1 in the case where $\frac{\sigma^{2}}{4}<\gamma$. (Note that there exists another steady state on the line $\{z=\sigma\}$ corresponding to the slow manifold $\mathcal{M}_{v}^{0}$; however, that state is not relevant for our analysis.) In panel 3(b), we assume $u=0$ in the proof of Lemma 4.2, whence the steady state at $(y, z)=(0, \sigma)$ is non-hyperbolic due to a transcritical bifurcation between the steady state at infinity and the slow manifold $\mathcal{M}_{v}^{0}$. Importantly, the independent variable has been reversed, i.e., the trapping region $\mathcal{S}$ is constructed in backward "time".

where $c_{u}$ and $c_{z}$ are again small and positive.

Lemma 4.2. Let $\kappa>0$, and consider $u^{\text {in }} \in\left[-c_{u}, c_{u}\right]$. Then, for $\epsilon=0$ in Equation (4.1), the intersection of the stable manifold of the steady state at $\left(0, z_{v}^{-}\right)$with the section $\Sigma^{\text {in }}$ can be represented as the graph $z=\sigma+H^{\mathrm{s}}\left(u^{\mathrm{in}}\right)$. Here, the function $H^{\mathrm{s}}\left(u^{\mathrm{in}}\right)$ is monotonically increasing. In the particular case of $u^{\mathrm{in}}=0, H^{\mathrm{s}}(0)$ has the expansion

$$
H^{\mathrm{s}}(0)=\frac{\gamma \kappa}{\alpha \sigma}+\mathcal{O}\left(\kappa^{2}\right)
$$

Proof: The existence of a heteroclinic orbit is shown as in the proof of Lemma 4.1. Consider $u^{\text {in }}=0$ initially. The steady state at $(y, z)=(0, \sigma)$ in $(4.2)$ is non-hyperbolic, with one unstable and one zero eigenvalue, which is due to a transcritical bifurcation of that state as it collides with the slow manifold $\mathcal{M}_{v}^{0}$. A trapping region $\mathcal{S}$ can be constructed that is similar to the one used in the proof of Lemma 4.1; to be precise, let $\mathcal{S}$ be bounded by the lines $\{z=\sigma\},\{v=0\}$, and $\left\{z=\sigma+\frac{\gamma}{\alpha \sigma} y\right\}$. It can then be verified that the flow of (4.1) points out of $\mathcal{S}$ on $\{z=\sigma\}$ and $\left\{z=\sigma+\frac{\gamma}{\alpha \sigma} y\right\}$, as well as that $\{v=0\}$ is invariant. Therefore, we may reverse the time-like independent variable in (4.1) and track the stable manifold of $(v, z)=\left(0, z_{v}^{-}\right)$backward, see Figure 3(b), which proves the existence of a heteroclinic connection between $\left(0, z_{v}^{-}\right)$and $(y, z)=\left(0, z_{y}^{-}\right)$. Furthermore, we see that this connection lies in the center manifold of the steady state at $\left(0, z_{y}^{-}\right)$, thus yielding the expansion in (4.5).

For $u^{\text {in }}<0$, the above heteroclinic connection between $\left(0, z_{v}^{-}\right)$and $\left(0, z_{y}^{-}\right)$persists, which can be shown by constructing another trapping region, with $z$ bounded between the heteroclinic corresponding to $u^{\text {in }}=0$ and some sufficiently large value of $z$. Finally, when $u^{\text {in }}>0$, a connection exists between $\left(0, z_{v}^{-}\right)$and $\left(\frac{u^{\text {in }} \alpha}{\gamma-u^{\text {in }}}, \sigma\right)$, the steady state corresponding to $\mathcal{M}_{v}^{0}$; here, the trapping region $\mathcal{S}$ is modified to the region with boundaries $\{z=\sigma\},\left\{y=\frac{u^{\text {in }} \alpha}{\gamma-u^{\text {in }}}\right\}$, and the heteroclinic found for $u^{\text {in }}=0$, which also implies monotonicity. 
In fact, considered as a steady state of Equation (4.2), the point $(u, y, z)=(0,0, \sigma)$ possesses a two-dimensional center manifold and a one-dimensional unstable manifold. For $\left|u^{\mathrm{in}}\right|$ small, heteroclinic orbits depart $\{y=0\}$ within that center manifold.

\subsection{Fast connections between $\mathcal{M}_{U}$ and $\mathcal{M}_{V}^{0}$}

Next, we consider the fast transition from $\mathcal{M}_{U}$ to $\mathcal{M}_{V}^{0}$. To that end, we recall Equation (3.6), where $\epsilon=0$, and the reduction thereof to the planar system in (3.7).

Lemma 4.3. Let $\Delta>0$. For any $\sigma<0$, there exists a heteroclinic orbit for Equation (3.7) that connects the steady states at $(0,0)$ and $\left(-\frac{\Delta}{\delta \sigma},-\frac{\Delta}{\delta}\right)$. For any $\sigma<-2 \sqrt{\Delta}$, that heteroclinic departs the origin along its weak unstable eigendirection.

Proof: With $\Delta>0$ and $\sigma<0$, the steady state at the origin in (3.7) is unstable, with eigenvalues $-\frac{\sigma}{2} \pm \frac{1}{2} \sqrt{\sigma^{2}-4 \Delta}$ and eigendirections $\mu_{ \pm}=\left(-\frac{\sigma}{2} \pm \frac{1}{2} \sqrt{\sigma^{2}-4 \Delta},-\Delta\right)^{T}$. Recall that, since $\sigma<0, \mu_{+}$is the strong unstable eigendirection, while $\mu_{-}$is the weak unstable one. The steady state at $\left(-\frac{\Delta}{\delta \sigma},-\frac{\Delta}{\delta}\right)$ is a saddle point, with eigenvalues $-\sigma>0$ and $\frac{\Delta}{\sigma}<0$. We restrict to the case of $\frac{\sigma^{2}}{4}>\Delta$ here, i.e., we only consider the scenario where the eigenvalues of the linearization about the origin in (3.7) are real.

The existence of a heteroclinic orbit then follows from a simple trapping region argument. Consider the triangular region $\mathcal{T}$ formed by the line $\left\{W=-\frac{\Delta}{\delta}\right\}$, the $V$-axis, and the weak eigendirection $\mu_{-}$, and note that $\mathcal{T}$ excludes the strong eigendirection $\mu_{+}$. The line $\left\{W=-\frac{\Delta}{\delta}\right\}$ is invariant under the flow of Equation (3.7). Moreover, it is immediate that the vector field in (3.7) points out of $\mathcal{T}$ along the $V$-axis, since $W<0$ there.

We hence turn our attention to the upper boundary of $\mathcal{T}$, which is given by the span of the weak unstable eigendirection. Again, it is convenient to introduce the projective coordinate $Z=\frac{W}{V}$ in Equation (3.7); thus,

$$
Z^{\prime}=-(\delta W+\Delta)-Z(Z-\sigma)
$$

When $W=0$, there exist two steady states $Z_{ \pm}=\frac{\sigma}{2} \pm \frac{1}{2} \sqrt{\sigma^{2}-4 \Delta}$; clearly, these states correspond to the eigendirections of the linearization of $(3.7)$ at $(0,0)$.

Note that, since $\sigma<Z_{-}<Z_{+}$, the steady state at $\left(-\frac{\Delta}{\delta \sigma},-\frac{\Delta}{\delta}\right)$ in (3.7) always lies at the bottom of $\mathcal{T}$. For any $(W, Z)$ with $Z=Z_{+}$or $Z=Z_{-}$, we find that $Z^{\prime}=-\delta W>0$. Hence, and since $W<0$, the vector field along the upper boundary of $\mathcal{T}$ points outside the region.

After reversal of the independent variable, $\mathcal{T}$ forms a trapping region, and we conclude the existence of a heteroclinic connection between the two steady states at $(0,0)$ and $\left(-\frac{\Delta}{\delta \sigma},-\frac{\Delta}{\delta}\right)$ in $(3.7)$. Finally, since that trapping region excludes the strong eigendirection, the heteroclinic connection cannot contain the strong unstable manifold of $(0,0)$ for $\frac{\sigma^{2}}{4}>\Delta$, i.e., when $\sigma<-2 \sqrt{\Delta}$.

Having shown the existence of heteroclinic connections between $\mathcal{M}_{U}$ and $\mathcal{M}_{V}^{0}$, we now derive some properties of these orbits that will be useful in Section 6. We recall that we will only employ $(U, V, W)$-coordinates when $V=\mathcal{O}(1)$, and we define the section

$$
\Delta^{\text {in }}=\left\{(U, V, Z) \mid U \in\left[\frac{\sigma^{2}}{4}-c_{u}, \frac{\sigma^{2}}{4}+c_{u}\right], V=\kappa, Z \in\left[\frac{\sigma}{2}-c_{Z}, \frac{\sigma}{2}+c_{Z}\right]\right\}
$$

in projectivized, rescaled coordinates, with $c_{u}$ and $c_{Z}$ small and positive; see Figure 2. 
Lemma 4.4. Let $\kappa>0$, and suppose that $\sigma$ satisfies $-\frac{\Delta}{\delta \kappa}<\sigma<0$. Consider the stable manifold of $\left(-\frac{\Delta}{\delta \sigma},-\frac{\Delta}{\delta}\right)$ in Equation (3.7) and its first intersection with the section $\Delta^{\mathrm{in}}$. Then, the resulting intersection point can be written as a graph $W=\kappa G^{\mathrm{s}}(\Delta)$ for $V>0$, where $G^{\mathrm{s}}(\Delta)$ is monotonically increasing.

Proof: The existence of a heteroclinic orbit follows from Lemma 4.3; for $\sigma>-\frac{\Delta}{\delta \kappa}$, that heteroclinic intersects the section $\Delta^{\text {in }}$. Again, we find it convenient to convert to projective coordinates in (3.7):

$$
\begin{aligned}
& V^{\prime}=V(Z-\sigma) \\
& Z^{\prime}=-\delta V Z-\Delta+\sigma Z-Z^{2} .
\end{aligned}
$$

Consider $\Delta=\Delta_{1}$ fixed, denote the tangent vector at any point $(V, Z)$ on the heteroclinic as $\left(t_{V}\left(V, Z, \Delta_{1}\right), t_{Z}\left(V, Z, \Delta_{1}\right)\right)^{T}$, and note that $t_{V}>0$ and $t_{Z}<0$. At that same point $(V, Z)$, calculate the vector field for some $\Delta$-value $\Delta_{2}>\Delta_{1}$ as $\left(t_{V}\left(V, Z, \Delta_{1}\right), t_{Z}\left(V, Z, \Delta_{1}\right)-\left(\Delta_{2}-\Delta_{1}\right)\right)^{T}$. Therefore, a trapping region can be constructed for $\Delta=\Delta_{2}$ that is based on the heteroclinic obtained for $\Delta_{1}$. It follows that the intersection point $G^{\mathrm{s}}(\Delta)$ is a monotonically increasing function of $\Delta$, as claimed.

\section{Proof of Theorem 1: setup and preliminaries}

We now turn our attention to the proof of Theorem 1. Consider the following section,

$$
\Sigma_{0}=\left\{(u, v, w) \mid u=u_{0}, u_{0}<\min \left\{\frac{\gamma}{2}, \frac{\sigma^{2}}{8}\right\}\right\} .
$$

We pursue a line of analysis developed in [35], where a very general result is presented on the existence of periodic orbits near sequences of slow manifolds. Their result requires the presence of fast orbits connecting the slow manifolds so that the stable or unstable foliations tracked along these orbits intersect transversely. That scenario is essentially realized here, with $\mathcal{M}_{V}^{0}$ and $\mathcal{M}_{u}$ playing the role of the slow manifolds. The different scalings of these two manifolds introduce complications that prevent the direct application of the main result in [35]. At the same time, we note that the three-dimensional nature of our problem allows for some simplification; in particular, we can follow manifolds directly, rather than tracking tangent spaces using projected volumes, as was done in [35].

Consider a two-dimensional subset $\mathcal{M}_{0} \subset \Sigma_{0}$ consisting of suitably chosen initial conditions that enter and exit a neighborhood of $\mathcal{M}_{u}$ near specified points. We will track these points forward and backward, and compare them in the section $\Sigma^{\text {in }}$; recall Equation (4.4). Specifically, let $\varphi_{\zeta}$ be the flow associated to the three-dimensional system, Equation (3.1), and define

$$
\mathcal{M}^{+}=\bigcup_{\zeta>0} \varphi_{\zeta}\left(\mathcal{M}_{0}\right) \quad \text { and } \quad \mathcal{M}^{-}=\bigcup_{\zeta<0} \varphi_{\zeta}\left(\mathcal{M}_{0}\right)
$$

as the forward and backward images of $\mathcal{M}_{0}$, respectively; we will show that these manifolds intersect for $\epsilon>0$ and that there exists a periodic orbit in their intersection. In GSPT, one typically tracks manifolds in the singular limit to show that they intersect transversely when $\epsilon=0$. If the manifolds perturb smoothly in $\epsilon$, one may then conclude the existence of a unique intersection for $\epsilon \neq 0$. We implement the same philosophy here, albeit with some modifications following [35]. Clearly, the 
manifolds $\mathcal{M}^{ \pm}$have dimension three for any $\epsilon>0$; however, to prove the existence of periodic orbits for Equation (1.1), it is not sufficient to show that these manifolds intersect in the section $\Sigma^{\text {in }}$, as orbits in $\mathcal{M}^{ \pm}$may have originated from different points. Rather, one also needs to track the initial condition corresponding to each orbit in $\mathcal{M}_{0}$ in order to ensure the existence of an intersection of the forward and backward images of $\mathcal{M}_{0}$ with the same initial condition. To that end, we will expand the vector field in (3.1) by appending two trivial equations that represent the initial conditions of each orbit in $\mathcal{M}_{0}$. We thus track the three-dimensional manifolds $\mathcal{M}^{ \pm}$in five-dimensional phase space. Now, a transverse intersection of these manifolds in $\Sigma^{\text {in }}$ for $\epsilon=0$ guarantees a unique solution with identical initial values in $\Sigma_{0}$ and, hence, a periodic orbit for Equation (1.1) when $\epsilon \neq 0$ is sufficiently small.

\subsection{Definition of $\mathcal{M}_{0}$}

In this subsection, we will specify the subset $\mathcal{M}_{0}$ of $\Sigma_{0}$ which will be the object of study going forward. The set $\mathcal{M}_{0}$ consists of those points whose orbits

1. enter a neighborhood of the manifold $\mathcal{M}_{u}$ close to the stable foliation $\mathcal{F}^{\mathrm{s}}\left(\mathcal{M}_{u}\right)$ at $u=0$; and

2. exit a neighborhood of the manifold $\mathcal{M}_{u}$ close to the (strong) unstable foliation $\mathcal{F}^{\mathrm{uu}}\left(\mathcal{M}_{u}\right)$ at $u=\frac{\sigma^{2}}{4}$.

The analysis outlined above will, in part, be performed in a Fenichel-type normal form which is valid on an appropriately defined neighborhood $\mathcal{N}\left(\mathcal{M}_{u}\right)$; cf. Lemma 6.1, the discussion in Appendix A.1, and Equation (A.3) below:

$$
\begin{aligned}
& \frac{d b}{d \tau}=\mu_{+}(u) b+\widetilde{B}(u, b, z, \epsilon) b \\
& \frac{d u}{d \tau}=\epsilon \\
& \frac{d z}{d \tau}=\mu_{-}(u) z+\widetilde{Z}(u, b, z, \epsilon) z^{2}
\end{aligned}
$$

here, $\tau$ is the new (scaled) independent variable, and

$$
\mu_{ \pm}(u)=\frac{-\sigma \pm \sqrt{\sigma^{2}-4(u-\gamma)}}{2 \delta(1-u)} .
$$

It follows that for all $0<u<\frac{\sigma^{2}}{4}+c_{u}$, there exists $\underline{\lambda}_{-}<0<\bar{\lambda}_{-}<\underline{\lambda}_{+}<\bar{\lambda}_{+}$such that $\underline{\lambda}_{ \pm}<$ $\mu_{ \pm}(u)<\bar{\lambda}_{ \pm}$. Any trajectory of (5.1) with initial condition $\left(b_{0}, u_{0}, z_{0}\right)$ that remains within $\mathcal{N}\left(\mathcal{M}_{u}\right)$ then satisfies the estimates

$$
\begin{gathered}
C_{b}^{-}\left|b_{0}\right| \mathrm{e}^{\lambda_{+} \tau} \leq|b(t)| \leq C_{b}^{+}\left|b_{0}\right| \mathrm{e}^{\bar{\lambda}_{+} \tau} \quad \text { for } \tau>0 \\
C_{z}^{-}\left|z_{0}\right| \mathrm{e}^{\lambda_{-} \tau} \leq|z(t)| \leq C_{z}^{+}\left|z_{0}\right| \mathrm{e}^{\bar{\lambda}_{-} \tau} \quad \text { for } \tau>0, \\
K_{b}^{+}\left|b_{0}\right| \mathrm{e}^{\bar{\lambda}_{+} \tau} \leq|b(t)| \leq K_{b}^{-}\left|b_{0}\right| \mathrm{e}^{\lambda_{+} \tau} \quad \text { for } \tau<0 \\
K_{z}^{+}\left|z_{0}\right| \mathrm{e}^{\bar{\lambda}_{-} \tau} \leq|z(t)| \leq K_{z}^{-}\left|z_{0}\right| \mathrm{e}^{\lambda_{-} \tau} \quad \text { for } \tau<0,
\end{gathered}
$$

for some positive constants $C_{\mathrm{b}}^{ \pm}, C_{\mathrm{z}}^{ \pm}, K_{\mathrm{b}}^{ \pm}$, and $K_{\mathrm{z}}^{ \pm}$. We are interested in orbits with initial $u$-value $u_{0}$ which enter $\mathcal{N}\left(\mathcal{M}_{u}\right)$ near $u=0$ and which then exit near $u=\frac{\sigma^{2}}{4}$. Let $c_{u}>0$ be chosen appropriately, and consider

$$
\left(u^{\text {in }}, u^{\text {out }}\right) \in \mathcal{I}=\left[-c_{u}, c_{u}\right] \times\left[\frac{\sigma^{2}}{4}-c_{u}, \frac{\sigma^{2}}{4}+c_{u}\right] \subset \mathbb{R}^{2} .
$$


We seek solutions of (5.1) with boundary conditions

$$
u(0)=u^{\text {in }}, \quad z(0)=\omega, \quad b(T)=\omega, \quad \text { and } \quad u(T)=u^{\text {out }}
$$

for some $\omega>0$, where $T=\frac{u^{\text {out }}-u^{\text {in }}}{\epsilon}$. The existence and uniqueness of such solutions is a consequence of [31, Theorem 2.1], and requires several conditions on $c_{u}$. Firstly, we restrict $c_{u}$ such that $\Pi_{0}\left(c_{u}\right)>\frac{\sigma^{2}}{4}$, which precludes the possibility of an orbit departing a neighborhood of the slow manifold near the center direction due to the loss-of-stability turning point. (Here, $\Pi_{0}$ denotes the Poincaré map that is associated with passage past $\mathcal{M}_{u}$; cf. Section A.2 for details.) Such a choice is possible, since the condition in (3.8) implies $\Pi_{0}(0)>\frac{\sigma^{2}}{4}$, and since $\Pi_{0}$ is a continuous map; see Remark 9 below. Secondly, we require $c_{u}$ sufficiently small for the assumptions in Lemmas 4.1 and 4.2 to remain satisfied.

Let $T^{+}=\frac{u^{\text {out }}-u_{0}}{\epsilon}$ and $T^{-}=\frac{u^{\text {in }}-u_{0}}{\epsilon}$, and denote the flow of (5.1) by $\varphi_{\tau}$. It then follows that

$$
\mathcal{M}_{0}=\left\{\left(b, u_{0}, z\right) \mid \varphi_{T^{+}}\left(b, u_{0}, z\right)=\left(\omega, u^{\text {out }}, z\left(T^{+}\right)\right), \varphi_{T^{-}}\left(b, u_{0}, z\right)=\left(b\left(T^{-}\right), u^{\text {in }}, \omega\right)\right\} .
$$

We will therefore parametrize the set $\mathcal{M}_{0}$ by $u^{\text {in }}$ and $u^{\text {out }}$, and will augment the vector field in Equation (3.1) accordingly:

$$
\begin{aligned}
u^{\prime} & =\frac{\epsilon \delta}{1-\epsilon \sigma}(1-u-u v) \\
v^{\prime} & =w-\sigma v \\
w^{\prime} & =-u v+\frac{\gamma v}{1+\alpha v} \\
\left(u^{\text {out }}\right)^{\prime} & =0 \\
\left(u^{\text {in }}\right)^{\prime} & =0 .
\end{aligned}
$$

We will typically consider $\mathcal{M}^{ \pm}$as three-dimensional manifolds in three-dimensional phase space, although we will revisit the five-dimensional system in Equation (5.5) to conclude the proof of Theorem 1.

\section{Proof of Theorem 1: existence of a transverse intersection}

Practically speaking, the forward tracking of $\mathcal{M}^{+}$under the flow of Equation (5.5) from its intersection with $\Sigma_{0}$ will be accomplished through a sequence of sections for the corresponding flow in various coordinate systems. In addition to the sections $\Sigma^{\text {out }}$ and $\Sigma^{\text {in }}$ defined in Equations (4.3) and (4.4), respectively, we will require two "intermediate" sections

$$
\begin{aligned}
\Delta^{\text {in }} & =\left\{(U, V, W) \mid U \in\left[\frac{\sigma^{2}}{4}-c_{u}, \frac{\sigma^{2}}{4}+c_{u}\right], V=\kappa, W \in\left[-c_{W}, c_{W}\right]\right\} \text { and } \\
\Delta^{\text {out }} & =\left\{(U, V, W) \mid U \in\left[-c_{u}, c_{u}\right], V=\kappa, W \in\left[-c_{W}, c_{W}\right]\right\},
\end{aligned}
$$

where $c_{u}$ and $c_{W}$ are small and positive constants, as before. We will then track $\mathcal{M}^{-}$backward from its intersection with the section $\Sigma_{0}$ to $\Sigma^{\text {in }}$ to establish the requisite transverse intersection.

We emphasize that the sections $\Sigma^{\text {out,in }}$ and $\Delta^{\text {in,out }}$ are defined in different coordinate systems, and that they are not equivalent. The tracking of orbits as they pass between these sections will require the use of blow-up, and will be performed in the coordinates of chart $K_{2}$; specifically, the forward tracking is accomplished in Sections 6.1 through 6.4, while the backward tracking is performed in Section 6.5. 


\subsection{The transition map $\Pi_{1}: \Sigma_{0} \rightarrow \Sigma^{\text {out }}$}

We begin by tracking the manifold $\mathcal{M}_{0}$ from $\Sigma_{0}$ to its intersection with $\Sigma^{\text {out }}$, which is achieved in two steps: firstly, we track $\mathcal{M}_{0}$ until it leaves a neighborhood of the slow manifold $\mathcal{M}_{u}$ by applying a generalization of the Exchange Lemma; secondly, the transition to large values of $v$ is described by tracking orbits of the reduced fast problem, as described in Lemma 4.1.

\subsubsection{Transition past $\mathcal{M}_{u}$}

When $\frac{\sigma^{2}}{4}<\gamma$, the slow manifold $\mathcal{M}_{u}$ is normally hyperbolic for $u<\frac{\sigma^{2}}{4}$; hence, the standard Exchange Lemma applies, see $[11,15]$. If $\gamma<\frac{\sigma^{2}}{4}$, the manifold $\mathcal{M}_{u}$ undergoes a loss-of-stability turning point at $u=\gamma$, in which case we require a generalized version of the Exchange Lemma.

We have the following result.

Lemma 6.1. Let $\epsilon \in\left(0, \epsilon_{0}\right)$, with $\epsilon_{0}>0$ sufficiently small, and let $\mathcal{K}_{u^{\text {in }}}$ denote the two-dimensional submanifold of $\mathcal{M}^{+}$with $u^{\text {in }} \in\left[-c_{u}, c_{u}\right]$ fixed. Then, the manifold $\mathcal{K}_{u^{\text {in }}}$ exits the neighborhood $\mathcal{N}\left(\mathcal{M}_{u}\right)$ near $u=\frac{\sigma^{2}}{4}$; moreover,

1. if $\frac{\sigma^{2}}{4}<\gamma$, the manifold $\mathcal{K}_{u^{\text {in }}}$ is $C^{1} \mathcal{O}(\epsilon)$-close to the unstable foliation $\mathcal{F}^{\mathrm{u}}\left(\mathcal{M}_{u}\right)$;

2. if $\gamma<\frac{\sigma^{2}}{4}$ and if the condition in (3.8) holds, $\mathcal{K}_{u^{\text {in }}}$ is $C^{1} \mathcal{O}(\epsilon)$-close to the strong unstable foliation $\mathcal{F}^{\mathrm{uu}}\left(\mathcal{M}_{u}\right)$ near $u^{\text {out }}=\frac{\sigma^{2}}{4}$.

Proof: The first statement is a direct consequence of the modified Exchange Lemma [11, 15, 35]. The second statement follows from a generalized Exchange Lemma and is proven in Appendix A.

Lemma 6.1 states that, upon leaving a neighborhood of $\mathcal{M}_{u}$, the manifold $\mathcal{M}^{+}$depends on $u^{\text {in }}$ only at higher orders in $\epsilon$. A similar statement holds regarding the dependence of $\mathcal{M}^{-}$on $u^{\text {out }}$.

\subsubsection{Transition from $\mathcal{M}_{u}$ to $\Sigma^{\text {out }}$}

Reverting from the Fenichel-type normal form in (A.1) to the original (unscaled) variables, we find that $\mathcal{M}^{+}$is $C^{1} \mathcal{O}(\epsilon)$-close to the (strong) unstable foliation $\mathcal{F}^{\mathrm{u}}\left(\mathcal{M}_{u}\right)\left(\mathcal{F}^{\mathrm{uu}}\left(\mathcal{M}_{u}\right)\right)$ which is, in turn, $C^{1} \mathcal{O}(\epsilon)$-close to the (strong) unstable eigendirection of (3.1) at $v=0$. We note that an $\mathcal{O}(1)$ amount of "time" is required for the tracked manifold $\mathcal{M}^{+}$to intersect the section $\Sigma^{\text {out}}$; Lemma 4.1 gives a leading-order description of that manifold within $\Sigma^{\text {out }}$. By standard results on the smooth dependence of solutions on parameters, we conclude that

$$
u=u^{\text {out }}+\epsilon \tilde{u}\left(u^{\text {in }}, u^{\text {out }}, \epsilon\right) \text { and } \quad w=\kappa H^{\text {uu }}\left(u^{\text {out }}\right)+\epsilon \kappa \tilde{w}\left(u^{\text {in }}, u^{\text {out }}, \epsilon\right),
$$

where $\tilde{u}$ and $\tilde{w}$, as well as their derivatives, are uniformly bounded in $\epsilon$.

\subsection{The transition map $\Pi_{2}: \Sigma^{\text {out }} \rightarrow \Delta^{\text {in }}$}

We now track the manifold $\mathcal{M}^{+}$as it evolves from $\Sigma^{\text {out }}$ to $\Delta^{\text {in }}$, noting that these two sections are defined in different coordinates. To approximate the corresponding transition map $\Pi_{2}$, we will utilize geometric desingularization and the coordinates of chart $K_{2}$; recall Equation (3.12).

We observe that, in the singular limit of $\epsilon=0$, Equation (3.12) admits two invariant subspaces, which are defined by $\left\{r_{2}=0\right\}$ and $\left\{\epsilon_{2}=0\right\}$. The dynamics within the subspace $\left\{r_{2}=0\right\}$ is 
determined by Equation (4.2) with $\epsilon=0$, while the dynamics in $\left\{\epsilon_{2}=0\right\}$ is equivalent to that of (3.5), again with $\epsilon=0$.

We also recall the two heteroclinic orbits that constitute the singular orbit $\Gamma$ in a neighborhood of the sections $\Sigma^{\text {out }}$ and $\Delta^{\text {in }}$. Specifically, in unscaled coordinates, Lemma 4.1 gives a heteroclinic which approaches infinity near $(u, z)=\left(\frac{\sigma^{2}}{4}, \frac{\sigma}{2}\right)$; in rescaled coordinates, Lemmas 4.3 and 4.4 yield the existence of a heteroclinic connection that departs $\mathcal{M}_{U}$ near $(U, Z)=\left(\frac{\sigma^{2}}{4}, \frac{\sigma}{2}\right)$. Transforming these heteroclinics to the coordinates of chart $K_{2}$, we observe that the contribution to $\Gamma$ in unscaled coordinates lies in the invariant subspace $\left\{r_{2}=0\right\}$, while the contribution in rescaled coordinates is located in $\left\{\epsilon_{2}=0\right\}$. Furthermore, both orbits converge to the steady state at $\left(u_{2}, r_{2}, w_{2}, \epsilon_{2}\right)=$ $\left(\frac{\sigma^{2}}{4}, 0, \frac{\sigma}{2}, 0\right)$, which is found in the intersection of the two subspaces; see Figure 4.

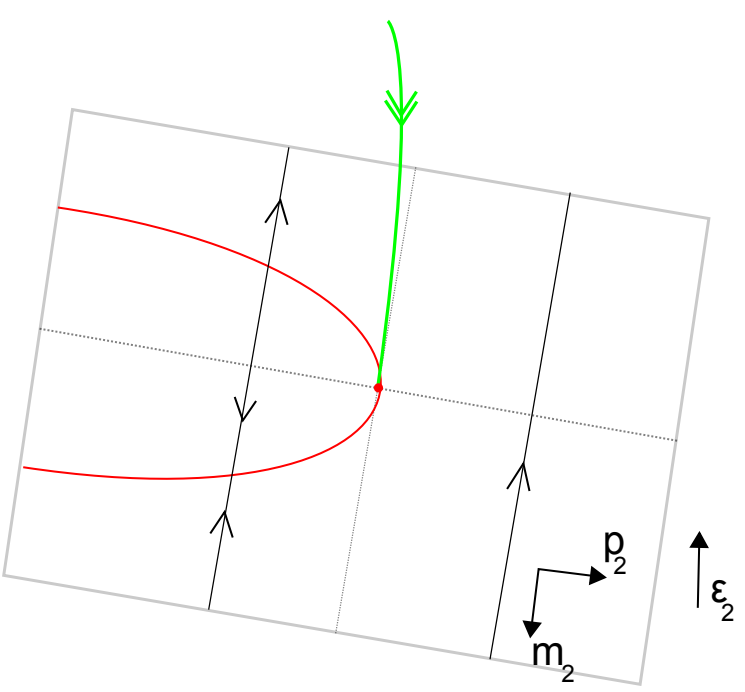

(a) Dynamics in $\left\{r_{2}=0\right\}$.

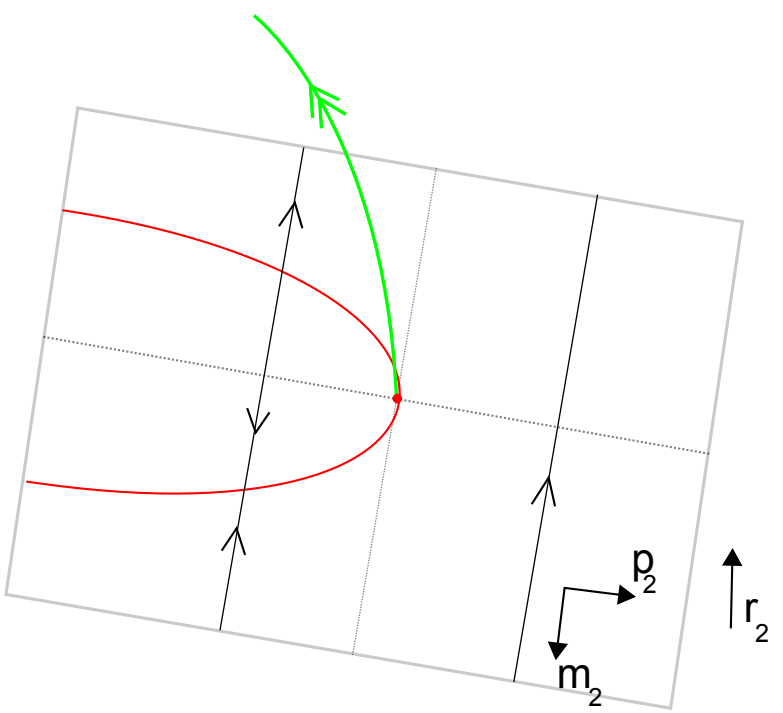

(b) Dynamics in $\left\{\epsilon_{2}=0\right\}$.

Figure 4: The dynamics of Equation (6.3) in the invariant subspaces $\left\{r_{2}=0\right\}$ and $\left\{\epsilon_{2}=0\right\}$. The flow in the center manifold $\mathcal{W}^{\mathrm{c}}(0)$ is prescribed by Equation (6.5); note the existence of a parabola of equilibria in $\mathcal{W}^{\mathrm{c}}(0)$. The singular orbit $\Gamma$ is depicted in green; within $\left\{r_{2}=0\right\}$ (panel $4(\mathrm{a})$ ), it consists of a heteroclinic connection from the steady state at $(v, z)=\left(0, z_{v}^{+}\right)$to that at the origin in $\left(p_{2}, r_{2}, m_{2}, \epsilon_{2}\right)$-coordinates, see also Figure $3(\mathrm{a})$. Within $\left\{\epsilon_{2}=0\right\}, \Gamma$ consists of a heteroclinic connection between the slow manifolds $\mathcal{M}_{U}$ and $\mathcal{M}_{V}^{0}$; again, that connection departs the origin in $\left(p_{2}, r_{2}, m_{2}, \epsilon_{2}\right)$-coordinates (panel $4(\mathrm{~b})$ ).

We need to track the passage of $\mathcal{M}^{+}$through a neighborhood of that steady state. To that end, we introduce new coordinates $p_{2}=u_{2}-\frac{\sigma^{2}}{4}$ and $m_{2}=w_{2}-\frac{\sigma}{2}$, shifting $\left(\frac{\sigma^{2}}{4}, 0, \frac{\sigma}{2}, 0\right)$ to the origin; then, the system in (3.12) becomes

$$
\begin{aligned}
p_{2}^{\prime} & =-\frac{\delta \sigma^{2}}{4} r_{2}-\delta r_{2} p_{2}+\epsilon_{2} r_{2} f\left(p_{2}+\frac{\sigma^{2}}{4}, r_{2}, r_{2} \epsilon_{2}\right) \\
r_{2}^{\prime} & =r_{2}\left(m_{2}-\frac{\sigma}{2}\right) \\
m_{2}^{\prime} & =-m_{2}^{2}-p_{2}+\epsilon_{2} \frac{\gamma}{\alpha+\epsilon_{2}} \\
\epsilon_{2}^{\prime} & =-\epsilon_{2}\left(m_{2}-\frac{\sigma}{2}\right) .
\end{aligned}
$$

Since we are interested in the flow for $\left|m_{2}\right|$ small, we can linearize the equations for $r_{2}$ and $\epsilon_{2}$ in 
(6.3) by a rescaling of the independent variable; Equation (6.3) then takes the form

$$
\begin{aligned}
p_{2}^{\prime} & =\frac{-\frac{\delta \sigma^{2}}{4} r_{2}-\delta r_{2} p_{2}+r_{2} \epsilon_{2} f\left(p_{2}+\frac{\sigma^{2}}{4}, r_{2}, r_{2} \epsilon_{2}\right)}{m_{2}-\frac{\sigma}{2}} \\
r_{2}^{\prime} & =r_{2} \\
m_{2}^{\prime} & =\frac{-m_{2}^{2}-p_{2}+\epsilon_{2} \frac{\gamma}{\alpha+\epsilon_{2}}}{m_{2}-\frac{\sigma}{2}} \\
\epsilon_{2}^{\prime} & =-\epsilon_{2} .
\end{aligned}
$$

We have the following result on the dynamics of (6.4) in a neighborhood of the origin.

Lemma 6.2. The origin is a steady state of Equation (6.4), with one positive eigenvalue 1, one negative eigenvalue -1 , and two zero eigenvalues. The associated stable and unstable eigendirections are given by $\chi_{\mathrm{s}}=\left(0,0, \frac{2 \gamma}{\alpha \sigma}, 1\right)^{T}$ and $\chi_{\mathrm{u}}=\left(\frac{\delta \sigma}{2}, 1, \delta, 0\right)^{T}$, respectively. Moreover, there exists $\delta_{\Omega}>0$ such that (6.4) has a smooth center manifold $\mathcal{W}^{\mathrm{c}}(0)$ that is defined in a neighborhood $\Omega \subset B_{\delta_{\Omega}}(0) \subset$ $\mathbb{R}^{4}$ of the origin. The manifold $\mathcal{W}^{\mathrm{c}}(0)$ can be taken to be the invariant subspace $\left\{r_{2}=0=\epsilon_{2}\right\}$; within $\Omega$, the reduced dynamics on $\mathcal{W}^{\mathrm{c}}(0)$ is described by

$$
\begin{aligned}
p_{2}^{\prime} & =0 \\
m_{2}^{\prime} & =-\frac{m_{2}^{2}+p_{2}}{m_{2}-\frac{\sigma}{2}} .
\end{aligned}
$$

In addition, the following statements hold.

1. There exists a smooth center-stable manifold $\mathcal{W}^{\mathrm{cs}}(0)$ which can be taken to be the invariant subspace $\left\{r_{2}=0\right\}$. The manifold $\mathcal{W}^{\mathrm{cs}}(0)$ can be decomposed into a union of stable fibers,

$$
\mathcal{W}^{\mathrm{cs}}(0)=\left\{x+c_{\mathrm{s}} \chi_{\mathrm{s}}+J_{\mathrm{s}}\left(c_{\mathrm{s}}, x\right) \mid x \in \mathcal{W}^{\mathrm{c}}(0)\right\},
$$

where $J_{\mathrm{s}}\left(c_{\mathrm{s}}, x\right): \mathbb{R} \times \mathcal{W}^{\mathrm{c}}(0) \rightarrow E_{\mathrm{c}}$ is $C^{1}$ and uniformly continuous. Moreover, $J_{\mathrm{s}}=\left(J_{\mathrm{s}}^{1}, 0, J_{\mathrm{s}}^{3}, J_{\mathrm{s}}^{4}\right)^{T}$, and there exists $K_{\mathrm{s}}\left(\delta_{\Omega}\right)>0$ such that $\left\|D J_{\mathrm{s}}\right\|<K_{\mathrm{s}} \delta_{\Omega}$.

2. A neighborhood of the center-stable manifold $\mathcal{W}^{\mathrm{cs}}(0)$ is foliated by unstable leaves $M_{\mathrm{u}}\left(x, x_{\mathrm{s}}\right)$, which can be written as the graph

$$
M_{\mathrm{u}}\left(x, x_{\mathrm{s}}\right)=\left\{x+x_{\mathrm{s}}+c_{\mathrm{u}} \chi_{\mathrm{u}}+J_{\mathrm{u}}\left(c_{\mathrm{u}}, x, x_{\mathrm{s}}\right) \mid\left(x, x_{\mathrm{s}}\right) \in \mathcal{W}^{\mathrm{cs}}(0)\right\}
$$

here, $J_{\mathrm{u}}\left(c_{\mathrm{u}}, x, x_{\mathrm{s}}\right): \mathbb{R} \times \mathcal{W}^{\mathrm{cs}}(0) \rightarrow E_{\mathrm{c}}$ is $C^{1}$ and uniformly continuous. Moreover, $J_{\mathrm{u}}=$ $\left(J_{\mathrm{u}}^{1}, J_{\mathrm{u}}^{2}, J_{\mathrm{u}}^{3}, 0\right)^{T}$, and there exists $K_{\mathrm{u}}\left(\delta_{\Omega}\right)>0$ such that $\left\|D J_{\mathrm{u}}\right\|<K_{\mathrm{u}} \delta_{\Omega}$.

Proof: The assertions concerning the existence of a center manifold and its invariant foliations follow from standard theory; see, for example, [3, 4, 38].

Next, we express the sections $\Sigma^{\text {out }}$ and $\Delta^{\text {in }}$ in the coordinates of chart $K_{2}$ :

$$
\Sigma^{\text {out }}=\left\{\left(p_{2}, r_{2}, m_{2}, \epsilon_{2}\right) \mid \epsilon_{2}=\kappa\right\} \quad \text { and } \quad \Delta^{\text {in }}=\left\{\left(p_{2}, r_{2}, m_{2}, \epsilon_{2}\right) \mid r_{2}=\kappa\right\},
$$

and we recall the rescaling of the independent variable which transformed Equation (6.3) into (6.4). In terms of that new independent variable, the transition "time" between $\Sigma^{\text {out }}$ and $\Delta^{\text {in }}$ is given explicitly by

$$
\widetilde{Z}=\ln \frac{\kappa}{r_{2}^{\text {in }}} .
$$


To track $\mathcal{M}^{+}$, we transform (6.2) to the coordinates of (6.3), wherein

$$
p_{2}=u^{\text {out }}-\frac{\sigma^{2}}{4}+\epsilon \tilde{u}\left(u^{\text {in }}, u^{\text {out }}, \epsilon\right), r_{2}=\frac{\epsilon}{\kappa}, m_{2}=H^{\text {uu }}\left(u^{\text {out }}\right)-\frac{\sigma}{2}+\epsilon \tilde{w}\left(u^{\text {in }}, u^{\text {out }}, \epsilon\right), \text { and } \epsilon_{2}=\kappa \text {. }
$$

We now proceed as follows: using Equation (6.6), we can decompose the manifold $\mathcal{M}^{+}$- or, rather, the representation thereof in the section $\Sigma^{\text {out }}$ - into the manifold of its base points within the center manifold $\mathcal{W}^{\mathrm{c}}(0)$ and the contribution from the corresponding stable fibers and unstable leaves. Since the flow in $\mathcal{W}^{\mathrm{c}}(0)$ reduces to a scalar differential equation with explicit solution, the base points can be tracked analytically. We have the following result.

Lemma 6.3. There exist positive constants $\kappa, \epsilon_{0}, c_{u}$, and $\delta_{\Omega}$ such that, for all $\epsilon \in\left[0, \epsilon_{0}\right)$ and $\left|u^{\text {out }}-\frac{\sigma^{2}}{4}\right|<c_{u}$, the manifold $\mathcal{M}^{+} \cap \Sigma^{\text {out }}$ can be expressed as

$$
\left(\begin{array}{c}
p_{\mathrm{b}} \\
m_{\mathrm{b}} \\
c_{\mathrm{s}} \\
c_{\mathrm{u}}
\end{array}\right)\left(u^{\text {in }}, u^{\text {out }}, \epsilon\right)=\left(\begin{array}{c}
P_{\mathrm{b}}\left(u^{\text {out }}\right)+\epsilon \widetilde{P}_{\mathrm{b}}\left(u^{\text {in }}, u^{\text {out }}, \epsilon\right) \\
L_{\mathrm{b}}\left(u^{\text {out }}\right)+\epsilon \widetilde{L}_{\mathrm{b}}\left(u^{\text {in }}, u^{\text {out }}, \epsilon\right) \\
C_{\mathrm{s}}^{\text {in }}\left(u^{\text {in }}, u^{\text {out }}, \epsilon\right) \\
C_{\mathrm{u}}^{\text {in }}\left(u^{\text {in }}, u^{\text {out }}, \epsilon\right)
\end{array}\right)
$$

in a neighborhood $\Omega \subset B_{\delta_{\Omega}}(0)$ of the origin in Equation (6.4). The functions $P_{\mathrm{b}}, \widetilde{P}_{\mathrm{b}}, L_{\mathrm{b}}, \widetilde{L}_{\mathrm{b}}, C_{\mathrm{s}}^{\mathrm{in}}$, and $C_{\mathrm{u}}^{\mathrm{in}}$ are at least $C^{1}-$ smooth, and can be expanded as

$$
\begin{aligned}
p_{\mathrm{b}}\left(u^{\text {in }}, u^{\text {out }}, \epsilon\right) & =u^{\text {out }}-\frac{\sigma^{2}}{4}+\left(\tilde{u}\left(0, \frac{\sigma^{2}}{4}, 0\right)-\frac{\delta \sigma}{2 \kappa}\right) \epsilon+\zeta_{p}\left(u^{\text {in }}, u^{\text {out }}, \epsilon\right) \\
m_{\mathrm{b}}\left(u^{\text {in }}, u^{\text {out }}, \epsilon\right) & =m^{*}+\frac{d H^{\text {uu }}}{d u^{\text {out }}}\left(\frac{\sigma^{2}}{4}\right)\left(u^{\text {out }}-\frac{\sigma^{2}}{4}\right)+\left(\frac{\delta}{\kappa}+\tilde{w}\left(0, \frac{\sigma^{2}}{4}, 0\right)\right) \epsilon+\zeta_{m}\left(u^{\text {in }}, u^{\text {out }}, \epsilon\right) \\
c_{\mathrm{S}}\left(u^{\text {in }}, u^{\text {out }}, \epsilon\right) & =c_{\mathrm{s}}^{*}+\zeta_{\mathrm{s}}\left(u^{\text {in }}, u^{\text {out }}, \epsilon\right) \\
c_{\mathrm{u}}\left(u^{\text {in }}, u^{\text {out }}, \epsilon\right) & =\frac{\epsilon}{\kappa}+\zeta_{\mathrm{u}}\left(u^{\text {in }}, u^{\text {out }}, \epsilon\right)
\end{aligned}
$$

for some values of $m^{*}$ and $c_{\mathrm{s}}^{*}$, where $\left|\zeta_{i}\right| \leq C\left[\delta_{\Omega}+\left(\left|u^{\text {out }}-\frac{\sigma^{2}}{4}\right|+\epsilon\right)^{2}\right]$ for some $C>0$.

Proof: The result is an application of the Implicit Function Theorem. We seek solutions of the following system of implicit equations,

$$
\left(\begin{array}{c}
p_{\mathrm{b}} \\
0 \\
m_{\mathrm{b}} \\
0
\end{array}\right)+c_{\mathrm{s}} \chi_{\mathrm{s}}+J_{\mathrm{s}}\left(c_{\mathrm{s}}, p_{\mathrm{b}}, m_{\mathrm{b}}\right)+c_{\mathrm{u}} \chi_{\mathrm{u}}+J_{\mathrm{u}}\left(c_{\mathrm{u}}, c_{\mathrm{s}}, p_{\mathrm{b}}, m_{\mathrm{b}}\right)-\left(\begin{array}{c}
u^{\text {out }}-\frac{\sigma^{2}}{4}+\epsilon \tilde{u}\left(u^{\text {in }}, u^{\text {out }}, \epsilon\right) \\
\frac{\epsilon}{\kappa} \\
H^{\mathrm{uu}}\left(u^{\text {out }}\right)-\frac{\sigma}{2}+\epsilon \tilde{w}\left(u^{\text {in }}, u^{\text {out }}, \epsilon\right) \\
\kappa
\end{array}\right)=0 .
$$

Let $\mathcal{F}\left(p_{\mathrm{b}}, m_{\mathrm{b}}, c_{\mathrm{s}}, c_{\mathrm{u}}, u^{\mathrm{in}}, u^{\text {out }}, \epsilon\right)$ denote the left-hand side in Equation (6.9). Note that $\mathcal{F}$ is at least $C^{1}$-smooth, since the functions $J_{\mathrm{s}}$ and $J_{\mathrm{u}}$ and the manifold $\mathcal{M}^{+}$are at least $C^{1}$, as well. We first consider $\epsilon=0$ and recall the proof of Lemma 4.1: there, it was shown that for $u^{\text {out }}=\frac{\sigma^{2}}{4}$, the point $(v, z)=\left(\frac{1}{\kappa}, H^{\mathrm{uu}}\left(\frac{\sigma^{2}}{4}\right)\right)$ converges to the non-hyperbolic point at infinity, i.e., to $(y, z)=$ $\left(0, \frac{\sigma}{2}\right)$. Transforming to center manifold coordinates, we find the existence of $m^{*}$ and $c_{\mathrm{s}}^{*}$ such that $\mathcal{F}\left(0, m^{*}, c_{\mathrm{s}}^{*}, 0, u^{\mathrm{in}}, \frac{\sigma^{2}}{4}, 0\right)=0$; see Figure 5 . We then calculate the Jacobian at that point,

$$
D \mathcal{F}_{\left(p_{\mathrm{b}}, m_{\mathrm{b}}, c_{\mathrm{s}}, c_{\mathrm{u}}\right)}\left(0, m^{*}, c_{\mathrm{s}}^{*}, 0, u^{*}, 0\right)=\left(\begin{array}{cccc}
e_{1} & e_{3} & \chi_{\mathrm{s}} & \chi_{\mathrm{u}}
\end{array}\right)+D J_{\mathrm{s}}+D J_{\mathrm{u}} .
$$

Let $A=\left(\begin{array}{llll}e_{1} & e_{3} & \chi_{\mathrm{s}} & \chi_{\mathrm{u}}\end{array}\right)$, and note that the matrix $A$ is invertible, since $\left\{e_{1}, e_{3}\right\}$ span the center eigenspace and $\chi_{s}$ and $\chi_{\mathrm{u}}$ span the stable and unstable spaces, respectively, at the origin in Equation 
(6.4); recall Lemma 6.2. Therefore, the above Jacobian is invertible for $\delta_{\Omega}$ sufficiently small, and the Implicit Function Theorem gives the existence of the $C^{1}$ solution stated in Equation (6.7). Next, let $\mathcal{G}(x, y)=x-D_{x} \mathcal{F}^{-1}\left(x^{*}\right) F(x, y)$, where we have introduced the shorthand notation $x=\left(p_{\mathrm{b}}, m_{\mathrm{b}}, c_{\mathrm{s}}, c_{\mathrm{u}}\right)$ and $y=\left(u^{\text {in }}, u^{\text {out }}, \epsilon\right)$. Clearly, the map $\mathcal{G}$ has a fixed point whenever $\mathcal{F}=0$. Exploiting $D_{x} \mathcal{F}^{-1}=A^{-1}+L$, with $\|L\|=\mathcal{O}\left(\delta_{\Omega}\right)$, we have

$$
\left(\begin{array}{c}
p_{\mathrm{b}} \\
m_{\mathrm{b}} \\
c_{\mathrm{s}} \\
c_{\mathrm{u}}
\end{array}\right)=-\left(A^{-1}+L\right)\left(D_{\left(u^{\text {in }}, u^{\text {out }}, \epsilon\right)} \mathcal{F}\left(x^{*}, y^{*}\right)+N(x, y)\right)
$$

where $N$ denotes nonlinear terms in $(x, y)$; hence,

$$
A^{-1}=\left(\begin{array}{cccc}
1 & -\frac{\delta \sigma}{2} & 0 & 0 \\
0 & -\delta & 1 & -\frac{2 \gamma}{\alpha \sigma} \\
0 & 0 & 0 & 1 \\
0 & 1 & 0 & 0
\end{array}\right) \quad \text { and } \quad D_{\left(u^{\text {in }}, u^{\text {out }}, \epsilon\right)} \mathcal{F}\left(x^{*}, y^{*}\right)=-\left(\begin{array}{ccc}
0 & 1 & \widetilde{P}^{\text {in }}\left(u^{*}, 0\right) \\
0 & 0 & \kappa^{-1} \\
0 & \frac{d M^{\text {in }}}{d u^{\text {out }}}\left(u^{*}\right) & \widetilde{M}^{\text {in }}\left(u^{*}, 0\right) \\
0 & 0 & 0
\end{array}\right) .
$$

From (6.10), we thus derive the expansions given in (6.8). (Note that $p_{\mathrm{b}}$ and $m_{\mathrm{b}}$ can naturally be represented as in $(6.7)$, by identifying $P_{\mathrm{b}}\left(u^{\text {out }}\right)=p_{\mathrm{b}}\left(u^{\text {out }}, 0\right)$ and $L_{\mathrm{b}}\left(u^{\text {out }}\right)=m_{\mathrm{b}}\left(u^{\text {out }}, 0\right)$. $)$

We now track the base points of the manifold $\mathcal{M}^{+}$as the corresponding orbit evolves from $\Sigma^{\text {out }}$ to $\Delta^{\text {in }}$. Since the transition "time" of the associated trajectory is known explicitly as a function of $\epsilon$, we can calculate the location of base points at the intersection of the tracked manifold with $\Delta^{\text {in }}$.

The flow in the center manifold $\mathcal{W}^{\mathrm{c}}(0)$ is given by Equation (6.5). We first undo the rescaling of the independent variable there, whence

$$
p_{2}^{\prime}=0 \quad \text { and } \quad m_{2}^{\prime}=-m_{2}^{2}-p_{2} .
$$

Restricting our attention to those orbits that remain within $\Omega$, we can expand the associated transition "time" as

$$
Z^{*}=\frac{\sigma}{2} \ln \frac{\epsilon}{\kappa^{2}} \theta_{\Omega}=\frac{\sigma \theta_{\Omega}}{2} \ln \epsilon-\sigma \theta_{\Omega} \ln \kappa,
$$

where $\theta_{\Omega}=1+\mathcal{O}\left(\delta_{\Omega}\right)$. The solution of Equation (6.11) with initial condition $\left(p_{2}, m_{2}\right)(0)$ is given by

$$
m_{2}(\zeta)=\sqrt{p_{2}(0)} \tan \left(-\sqrt{p_{2}(0)} \zeta+c\right),
$$

where $c=\tan ^{-1} \frac{m_{2}(0)}{\sqrt{p_{2}(0)}}$. We are interested in those initial values for which the $m_{2}$-component remains bounded inside $\Omega$ once the independent variable has reached $Z^{*}$, which imposes a restriction on the admissible values for $p_{2}(0)$. Writing $\phi(\epsilon)=(\ln \epsilon)^{-1}$, we expand variables as

$$
Z^{*}=\frac{Z_{0}}{\phi}+Z_{1}, \quad \sqrt{p_{2}(0)}=\pi_{1} \phi+\pi_{2} \phi^{2}, \quad c=\frac{\pi}{2}+c_{1} \phi, \quad \text { and } \quad m_{2}(0)=m_{0}+m_{1} \phi .
$$

Substituting first into the equation for the initial value, we find that

$$
c_{1}=-\frac{\pi_{1}}{m_{0}} .
$$


Next, we substitute into the solution, Equation (6.12), and we expand the argument of the tangent function therein:

$$
m^{\text {out }}=\left(\pi_{1} \phi+\pi_{2} \phi^{2}\right) \tan \left[\frac{\pi}{2}-\pi_{1} Z_{0}+\left(c_{1}-Z_{1} \pi_{1}-Z_{0} \pi_{2}\right) \phi+\mathcal{O}\left(\phi^{2}\right)\right] .
$$

As we are interested in $m^{\text {out }}<0$, we require the leading-order terms in the above relation to satisfy $\frac{\pi}{2}-\pi_{1} Z_{0}=-\frac{\pi}{2}$, whence

$$
\pi_{1}=\frac{\pi}{Z_{0}}
$$

We now consider those base points for which $p_{\mathrm{b}}=\frac{\pi}{Z_{0}} \phi+\pi_{2} \phi^{2}$. Lemma 6.3 allows us to solve for $u^{\text {out }}$ as a function of $\pi_{2}$, and thus to express $m_{\mathrm{b}}$ as a function of $\pi_{2}$, as well. Consequently, $m^{\text {out }}$ is given by Equation (6.12), with leading-order expansion

$$
m^{\text {out }}=\frac{\pi_{1}}{c_{1}-Z_{1} \pi_{1}-Z_{0} \pi_{2}}=-\frac{1}{Z_{0}^{2} \pi_{2} \pi^{-1}+Z_{1}+m_{0}^{-1}},
$$

We have therefore established the following result.

Lemma 6.4. Let $\epsilon \in\left(0, \epsilon_{0}\right)$, with $\epsilon_{0}>0$ sufficiently small. Within the section $\Delta^{\text {in }}$, the manifold $\mathcal{M}^{+}$can then be expressed in terms of the center manifold coordinates $\left(p_{\mathrm{b}}, m_{\mathrm{b}}, c_{\mathrm{s}}, c_{\mathrm{u}}\right)$, with base points defined as

$$
p_{\mathrm{b}}=\frac{\pi}{Z_{0}} \phi(\epsilon)+\pi_{2}\left(u^{\mathrm{in}}, u^{\mathrm{out}}, \epsilon\right) \phi(\epsilon)^{2} \quad \text { and } \quad m_{\mathrm{b}}=m^{\mathrm{out}}\left(\pi_{2}, \phi(\epsilon), u^{\mathrm{in}}, u^{\mathrm{out}}, \epsilon\right),
$$

where $\phi(\epsilon)=(\ln \epsilon)^{-1}$ and $m^{\text {out }}$ is defined as in Equation (6.14).

In particular, we note the appearance of logarithmic switchback terms in Equation (6.14).



Figure 5: The dynamics of Equation (6.5), illustrating Lemma 6.7. Dashed lines represent the coordinate axes; the quadratic curve of steady states within the center manifold $\mathcal{W}^{c}(0)$, given by $\left\{p_{2}=-m_{2}^{2}\right\}$, is depicted in black. Lines with $p_{2}$ constant are invariant; the flow on one such line is shown. The black curve represents the base points of the manifold $\mathcal{M}^{+} \cap \Sigma^{\text {out }}$ in $\mathcal{W}^{\mathrm{c}}(0)$, which are evolved under the flow of (6.5) until the corresponding orbits reach the section $\Delta^{\text {in }}$. The curve of base points of the manifold $\mathcal{F}^{\mathrm{s}}\left(\mathcal{M}_{V}^{\epsilon}\right) \cap \Delta^{\text {in }}$ is depicted in solid grey; the $m_{\mathrm{b}}$-value $m^{*}$ defined in Lemma 6.3 is also shown.

Remark 8. In Section $\Delta^{\text {in }}$, the dependence of the manifold $\mathcal{M}^{+}$on $u^{\text {in }}$ is of higher order in $\epsilon$. For that reason, we may think of $\mathcal{M}^{+}$as depending on $u^{\text {out }}$ alone, to the order considered here. 


\subsection{The transition map $\Pi_{3}: \Delta^{\text {in }} \rightarrow \Delta^{\text {out }}$}

Next, we track $\mathcal{M}^{+}$as it undergoes a fast transition to the slow manifold $\mathcal{M}_{V}^{\epsilon}$, and as it then evolves slowly towards the section $\Delta^{\text {out}}$; our aim is to derive an expression for $\mathcal{M}^{+}$within that section.

We first collect several facts regarding the slow manifold $\mathcal{M}_{V}^{\epsilon}$ and its stable and unstable foliations. The stable foliation of $\mathcal{M}_{V}^{\epsilon}$ is then tracked in backward "time" to $\Delta^{\text {in }}$, where we establish

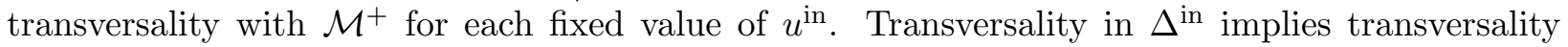
overall as the manifold $\mathcal{M}^{+}$enters a neighborhood of $\mathcal{M}_{V}^{\epsilon}$. Finally, we apply the Exchange Lemma to conclude that $\mathcal{M}^{+}$is $C^{1}$ exponentially close to the unstable foliation of $\mathcal{M}_{V}^{\epsilon}$ in $\Delta^{\text {out }}$, again for $u^{\text {in }}$ fixed.

\subsubsection{The slow manifold $\mathcal{M}_{V}^{\epsilon}$}

Standard GSPT [11, 15] implies the following result on the flow of Equation (3.5) on the slow manifold $\mathcal{M}_{V}^{\epsilon}$ for $\epsilon \neq 0$ :

Lemma 6.5. There exists $V_{*}>0$ and $\epsilon_{0}>0$ sufficently small such that for all $V>V_{*}$ and $\epsilon \in\left(0, \epsilon_{0}\right)$, the normally hyperbolic manifold $\mathcal{M}_{V}^{0}$ perturbs to an invariant manifold $\mathcal{M}_{V}^{\epsilon}$ which is $\mathcal{O}(\epsilon)$-close to $\mathcal{M}_{V}^{0}$. The manifold $\mathcal{M}_{V}^{\epsilon}$ can be expressed as a graph over $V$, with asymptotic expansion

$$
\begin{aligned}
U(V, \epsilon) & =\frac{\epsilon}{V}+\epsilon^{2}\left(\frac{\gamma-\alpha}{\delta \sigma \alpha V^{3}}-\frac{1}{V^{2}}\right)+\mathcal{O}\left(\epsilon^{3}\right) \quad \text { and } \\
W(V, \epsilon) & =\sigma V+\epsilon \frac{\gamma-\alpha}{\alpha \sigma}+\epsilon^{2}\left(\frac{\alpha^{2}-\gamma}{\alpha^{2} \sigma V}-\frac{\gamma-\alpha}{\delta \sigma^{2} \alpha V^{2}}\right)+\mathcal{O}\left(\epsilon^{3}\right) .
\end{aligned}
$$

Associated to $\mathcal{M}_{V}^{\epsilon}$ are invariant stable and unstable foliations $\mathcal{F}^{\mathrm{s}}\left(\mathcal{M}_{V}^{\epsilon}\right)$ and $\mathcal{F}^{\mathrm{u}}\left(\mathcal{M}_{V}^{\epsilon}\right)$, respectively, which are given as graphs over the stable and unstable eigenspaces of $\mathcal{M}_{V}^{0}$, respectively. Moreover, $\mathcal{F}^{\mathrm{s}}\left(\mathcal{M}_{V}^{\epsilon}\right)$ and $\mathcal{F}^{\mathrm{u}}\left(\mathcal{M}_{V}^{\epsilon}\right)$ are $\mathcal{O}(\epsilon)$-close, and diffeomorphic to, the stable and unstable manifolds of $\mathcal{M}_{V}^{0}$, respectively.

\subsubsection{Tracking $\mathcal{F}^{\mathrm{s}}\left(\mathcal{M}_{V}^{\epsilon}\right)$ backward to $\Delta^{\text {in }}$}

The stable foliation $\mathcal{F}^{\mathrm{s}}\left(\mathcal{M}_{V}^{\epsilon}\right)$ is $\mathcal{O}(\epsilon)$-close to the family of heteroclinic orbits constructed in Lemma 4.3. By the smooth dependence of Equation (3.5) on its parameters and initial conditions, we may track $\mathcal{F}^{\mathrm{s}}\left(\mathcal{M}_{V}^{\epsilon}\right)$ to $\Delta^{\text {in }}$, where it can be represented as

$$
U=\Delta+\delta \kappa G^{\mathrm{s}}(\Delta)+\epsilon \widetilde{U}(\Delta, \epsilon) \text { and } W=\kappa G^{\mathrm{s}}(\Delta)+\epsilon \kappa \widetilde{W}(\Delta, \epsilon) .
$$

Transforming to the coordinates of (6.4), we find

$$
u_{2}=\Delta-\frac{\sigma^{2}}{4}+\delta \kappa G^{\mathrm{S}}(\Delta)+\epsilon \widetilde{U}(\Delta, \epsilon), r_{2}=\kappa, m_{2}=G^{\mathrm{S}}(\Delta)-\frac{\sigma}{2}+\epsilon \widetilde{W}(\Delta, \epsilon) \text {, and } \epsilon_{2}=\frac{\epsilon}{\kappa} .
$$

To compare the above expression with $\mathcal{M}^{+}$, we transform it to the center manifold coordinates defined in Lemma 6.4. Hence, we obtain expressions for the corresponding base points in terms of $\Delta$ and $\epsilon$. 
Lemma 6.6. There exist positive constants $\kappa, \epsilon_{0}, \Delta_{0}$, and $\delta_{\Omega}$ such that, for all $\epsilon \in\left[0, \epsilon_{0}\right)$ and all $\left|\Delta-\frac{\sigma^{2}}{4}\right|<\Delta_{0}$, the manifold $\mathcal{F}^{\mathrm{s}}\left(\mathcal{M}_{V}^{\epsilon}\right) \cap \Delta^{\text {in }}$ can be expressed as

$$
\left(\begin{array}{c}
p_{\mathrm{b}} \\
m_{\mathrm{b}} \\
c_{\mathrm{s}} \\
c_{\mathrm{u}}
\end{array}\right)(\Delta, \epsilon)=\left(\begin{array}{c}
Q_{\mathrm{b}}(\Delta)+\epsilon \widetilde{Q}_{\mathrm{b}}(\Delta, \epsilon) \\
M_{\mathrm{b}}(\Delta)+\epsilon \widetilde{M}_{\mathrm{b}}(\Delta, \epsilon) \\
C_{\mathrm{s}}^{\text {out }}(\Delta, \epsilon) \\
C_{\mathrm{u}}^{\text {out }}(\Delta, \epsilon)
\end{array}\right)
$$

in a neighborhood of $\Omega \subset B_{\delta_{\Omega}}(0)$ of the origin in Equation (6.4), with expansions

$$
\begin{aligned}
p_{\mathrm{b}}(\Delta, \epsilon) & =\left(1+\delta \kappa \frac{d G^{s}}{d \Delta}\left(\frac{\sigma^{2}}{4}\right)\right)\left(\Delta-\frac{\sigma^{2}}{4}\right)+\widetilde{U}\left(\frac{\sigma^{2}}{4}, 0\right) \epsilon+\eta_{p}(\Delta, \epsilon) \\
m_{\mathrm{b}}(\Delta, \epsilon) & =m^{*}+\frac{d G^{\mathrm{s}}}{d \Delta}\left(\frac{\sigma^{2}}{4}\right)\left(\Delta-\frac{\sigma^{2}}{4}\right)+\left(-\frac{2 \gamma}{\delta \sigma \kappa}+\widetilde{W}\left(\frac{\sigma^{2}}{4}, 0\right)\right) \epsilon+\eta_{m}(\Delta, \epsilon) \\
c_{\mathrm{S}}(\Delta, \epsilon) & =\frac{\epsilon}{\kappa}+\eta_{\mathrm{s}}(\Delta, \epsilon) \\
c_{\mathrm{u}}(\Delta, \epsilon) & =c_{\mathrm{u}}^{*}+\eta_{\mathrm{u}}(\Delta, \epsilon)
\end{aligned}
$$

here, $\left|\eta_{i}\right|<C\left[\delta_{\Omega}+\left(\left|\Delta-\Delta^{*}\right|+\epsilon\right)^{2}\right]$. (Again, the functions $Q_{\mathrm{b}}, \widetilde{Q}_{\mathrm{b}}, M_{\mathrm{b}}, \widetilde{M}_{\mathrm{b}}, C_{\mathrm{s}}^{\text {out}}$, and $C_{\mathrm{u}}^{\text {out }}$ are at least $C^{1}$-smooth, with $m^{*}$ and $c_{\mathrm{u}}^{*}$ determined from the singular limit in (6.4).)

Proof: The proof is analogous to that of Lemma 6.3, and is hence omitted.

\subsubsection{Transverse intersection with $\mathcal{F}^{\mathrm{s}}\left(\mathcal{M}_{V}^{\epsilon}\right)$}

We now compare the expressions in Equations (6.15) and (6.17). For the moment, we suppress the dependence of $(6.15)$ on $u^{\text {in }}$ and $u^{\text {out }}$, and consider the expansions therein as functions of $\pi_{2}$. We obtain the following result.

Lemma 6.7. Let $\epsilon \in\left(0, \epsilon_{0}\right)$, with $\epsilon_{0}>0$ sufficiently small. Then, the base points of $\mathcal{M}^{+}$and $\mathcal{F}^{\mathrm{s}}\left(\mathcal{M}_{V}^{\epsilon}\right)$ intersect for those values of $\left(\Delta, \pi_{2}, \epsilon\right)$ for which

$$
\begin{aligned}
Q_{\mathrm{b}}(\Delta)+\epsilon \widetilde{Q}_{\mathrm{b}}(\Delta, \epsilon) & =\frac{\pi}{Z_{0}} \phi(\epsilon)+\pi_{2} \phi(\epsilon)^{2} \\
M_{\mathrm{b}}(\Delta)+\epsilon \widetilde{M}_{\mathrm{b}}(\Delta, \epsilon) & =m^{\text {out }}\left(\pi_{2}, \phi(\epsilon), \epsilon\right) .
\end{aligned}
$$

The above set of implicit equations has a smooth solution, with expansion

$$
\Delta=\frac{\sigma^{2}}{4}+\frac{\pi \phi(\epsilon)}{Z_{0} Q_{\mathrm{b}}^{\prime}\left(\frac{\sigma^{2}}{4}\right)}+\mathcal{O}\left(\phi^{2}\right) \quad \text { and } \quad \pi_{2}=-\frac{\pi}{Z_{0}^{2}}\left(\frac{1}{M_{\mathrm{b}}\left(\frac{\sigma^{2}}{4}\right)}+Z_{1}+\frac{1}{m_{0}}\right)+\mathcal{O}(\phi) .
$$

Proof: We apply the Implicit Function Theorem. Lemma 6.3 and Lemma 6.6 imply that the base points $\left(p_{\mathrm{b}}, m_{\mathrm{b}}\right)$ comprise smooth manifolds themselves. We consider Equation (6.19) as a function of $\left(\Delta, \pi_{2}, \phi\right)$, noting the existence of $\pi_{2}^{*}$ for which $\left(\frac{\sigma^{2}}{4}, \pi_{2}^{*}, 0\right)$ is a solution of Equation (6.19). The Jacobian evaluated at that point is

$$
\left(\begin{array}{cc}
\frac{d Q_{\mathrm{b}}}{d \Delta}\left(\frac{\sigma^{2}}{4}\right) & 0 \\
\frac{d M_{\mathrm{b}}}{d \Delta}\left(\frac{\sigma^{2}}{4}\right) & -\frac{\partial m^{\text {out }}}{\partial \pi_{2}}
\end{array}\right)
$$

whence Lemma 6.3 implies $Q_{\mathrm{b}}^{\prime} \neq 0$. One can verify that $\frac{\partial m^{\text {out }}}{\partial \pi_{2}} \neq 0$ by a direct calculation. 
To summarize, we have found expressions for the base points of $\mathcal{M}^{+} \cap \Delta^{\text {in }}$ and $\mathcal{F}^{\mathrm{s}}\left(\mathcal{M}_{V}^{\epsilon}\right) \cap \Delta^{\text {in }}$, as well as the points of intersection of these two curves, for each $\epsilon \in\left[0, \epsilon_{0}\right)$. Note that $\pi_{2}$ can be related to $u^{\text {in,out }}$ through $(6.13)$, and that we can solve for $u^{\text {out }}$ as a function of $u^{\text {in }}$, at such intersection points.

In fact, we are interested in intersections of the full manifolds $\mathcal{M}^{+} \cap \Delta^{\text {in }}$ and $\mathcal{F}^{\mathrm{s}}\left(\mathcal{M}_{V}^{\epsilon}\right) \cap \Delta^{\text {in }}$, rather than merely in their base points. Define $\Upsilon: \mathbb{R}^{4} \rightarrow \mathcal{W}^{\mathrm{c}}(0)$ to be the projection that associates points in $\mathbb{R}^{4}$ with their base points in the center manifold $\mathcal{W}^{\mathrm{c}}(0)$. We know that, by construction, the base points studied in Lemma 6.7 correspond to orbits lying in the section $\Delta^{\text {in }}$. Note that, since $\Upsilon: \Delta^{\text {in }} \rightarrow \mathcal{W}^{\mathrm{c}}(0)$ is not bijective, it is not a priori true that intersection of base points within $\mathcal{W}^{\mathrm{c}}(0)$ implies intersection of the full manifolds in $\Delta^{\text {in }}$. However, given the relationship between $r_{2}$ and $\epsilon_{2}$, with $r_{2} \epsilon_{2}=\epsilon$, we know that $\epsilon_{2}=\frac{\epsilon}{\kappa}$ within $\Delta^{\text {in }}$. For $\epsilon$ fixed, we hence define the submanifold $\widetilde{\Delta}=\left\{\left(p_{2}, r_{2}, m_{2}, \epsilon_{2}\right) \mid r_{2}=\kappa, \epsilon_{2}=\frac{\epsilon}{\kappa}\right\}$ of $\Delta^{\text {in }}$; then, the restriction $\Upsilon: \widetilde{\Delta} \rightarrow \mathcal{W}^{\mathrm{c}}(0)$ is bijective for each $\epsilon$, and we find that $\mathcal{M}^{+} \cap \Delta^{\text {in }}$ and $\mathcal{F}^{\mathrm{s}}\left(\mathcal{M}_{V}^{\epsilon}\right) \cap \Delta^{\text {in }}$ also intersect.

Finally, we note that Lemma 6.7 shows that the manifolds of base points intersect transversely as a function of $\pi_{2}$. Reverting to $(U, V, W)$-coordinates and recalling the relationship between $\pi_{2}$ and $u^{\text {in,out}}$, we find that $\mathcal{M}^{+}$intersects transversely with $\mathcal{F}^{\mathrm{s}}\left(\mathcal{M}_{V}^{\epsilon}\right)$ for each fixed $u^{\text {in }}$.

\subsubsection{Expression for $\mathcal{M}^{+} \cap \Delta^{\text {out }}$}

We now track $\mathcal{M}^{+}$from $\Delta^{\text {in }}$ to $\Delta^{\text {out }}$. For $u^{\text {in }}$ fixed, we have transversality of the tracked manifold with $\mathcal{F}^{\mathrm{s}}\left(\mathcal{M}_{V}^{\epsilon}\right)$ in $\Delta^{\text {in }}$; this transversality persists as the tracked manifold enters a neighborhood of $\mathcal{M}_{V}^{\epsilon}$. The Exchange Lemma then gives an expression for that manifold within $\Delta^{\text {out }}$. We have the following result.

Lemma 6.8. For $\epsilon \in\left(0, \epsilon_{0}\right)$ with $\epsilon_{0}>0$ sufficiently small and each fixed $u^{\text {in }} \in\left[-c_{u}, c_{u}\right]$, the manifold $\mathcal{M}^{+} \cap \Delta^{\text {out }}$ is $C^{1} \mathcal{O}\left(\mathrm{e}^{-\frac{C}{\epsilon}}\right)$-close to the unstable foliation $\mathcal{F}^{\mathrm{u}}\left(\mathcal{M}_{V}^{\epsilon}\right)$.

\subsection{The transition map $\Pi_{4}: \Delta^{\text {out }} \rightarrow \Sigma^{\text {in }}$}

We now track the manifold $\mathcal{M}^{+}$from $\Delta^{\text {out }}$ to $\Sigma^{\text {in }}$. Since these two sections are again defined in different coordinates, we must apply geometric desingularization to describe the evolution of $\mathcal{M}^{+}$ there.

To that end, we will continue the slow manifold $\mathcal{M}_{V}^{\epsilon}$ and its stable and unstable foliations $\mathcal{F}^{\mathrm{s}}\left(\mathcal{M}_{V}^{\epsilon}\right)$ and $\mathcal{F}^{\mathrm{u}}\left(\mathcal{M}_{V}^{\epsilon}\right)$ from $V=\kappa$ to $V=\mathcal{O}(\epsilon)$. Recall that, by Lemma 6.5 , these foliations are only defined for $V>V_{*}$, with $V_{*}=\mathcal{O}(1)$. At the heart of the matter lies the resolution of the dynamics near the non-hyperbolic origin in $(U, V, W, \epsilon)$-space, where $\mathcal{M}_{V}^{0}$ and $\mathcal{M}_{U}$ intersect.

We will perform our analysis in the coordinates of chart $K_{2}$; recall Equation (3.12). As was the case in Section 6.2, the subspaces $\left\{r_{2}=0\right\}$ and $\left\{\epsilon_{2}=0\right\}$ are invariant. Furthermore, we find three curves of equilibria for Equation (3.12),

$$
\begin{aligned}
& l_{1}=\left\{\left(0, r^{*}, \sigma, 0\right), r^{*} \geq 0\right\}, \\
& l_{2}=\left\{\left(u^{*}, 0, \frac{\sigma}{2}+\frac{1}{2} \sqrt{\sigma^{2}-4 u^{*}}, 0\right), u^{*} \leq \frac{\sigma^{2}}{4}\right\}, \quad \text { and } \\
& l_{3}=\left\{\left(\frac{\epsilon^{*} \gamma}{\alpha+\epsilon^{*}}, 0, \sigma, \epsilon^{*}\right), \epsilon^{*} \geq 0\right\} .
\end{aligned}
$$

These curves collide at the point $\left(u_{2}, r_{2}, w_{2}, \epsilon_{2}\right)=(0,0, \sigma, 0)$. Note that $l_{1}$ equals the singular slow manifold $\mathcal{M}_{V}^{0}$, while $l_{2}$ is a curve of equilibria at infinity; finally, $l_{3}$ represents the reduced slow 
manifold $\mathcal{M}_{v}^{0}$ in the unscaled $(u, v, w)$-coordinates. To track the slow manifold $\mathcal{M}_{V}^{\epsilon}$ and its unstable foliation $\mathcal{F}^{\mathrm{u}}\left(\mathcal{M}_{V}^{\epsilon}\right)$ as they pass the steady state at $(0,0, \sigma, 0)$, we require further information on the flow in a neighborhood of that point.

The state at $(0,0, \sigma, 0)$ is non-hyperbolic, with three zero eigenvalues pertaining to the curves $l_{j}(j=1,2,3)$ and one positive eigenvalue given by $-\sigma$. For convenience, we first shift $(0,0, \sigma, 0)$ to the origin by introducing the new variable $z_{2}=w_{2}-\sigma$, wherein Equation (3.12) assumes the form

$$
\begin{aligned}
u_{2}^{\prime} & =-\delta r_{2} u_{2}+r_{2} \epsilon_{2} f\left(u_{2}, r_{2}, r_{2} \epsilon_{2}\right) \\
r_{2}^{\prime} & =r_{2} z_{2} \\
z_{2}^{\prime} & =-z_{2}\left(z_{2}+\sigma\right)+\epsilon_{2} \frac{\gamma}{\alpha+\epsilon_{2}}-u_{2} \\
\epsilon_{2}^{\prime} & =-\epsilon_{2} z_{2} .
\end{aligned}
$$

Next, we diagonalize the linear part in (6.20) via the transformation

$$
\left(\begin{array}{l}
u_{2} \\
r_{2} \\
z_{2} \\
\epsilon_{2}
\end{array}\right)=\left(\begin{array}{rrrr}
\sigma & 0 & 0 & \frac{\gamma}{\alpha} \\
0 & 1 & 0 & 0 \\
-1 & 0 & 1 & 0 \\
0 & 0 & 0 & 1
\end{array}\right)\left(\begin{array}{c}
\hat{u} \\
\hat{r} \\
\hat{z} \\
\hat{\epsilon}
\end{array}\right),
$$

whence

$$
\begin{aligned}
\hat{u}^{\prime} & =-\delta \hat{r} \hat{u}+\hat{\epsilon}\left(-\frac{\delta \gamma}{\alpha \sigma} \hat{r}+\frac{\hat{r}}{\sigma} \tilde{f}(\hat{u}, \hat{r}, \hat{\epsilon})+\frac{\gamma}{\alpha \sigma} \hat{z}-\frac{\gamma}{\alpha \sigma} \hat{u}\right) \\
\hat{r}^{\prime} & =\hat{r}(\hat{z}-\hat{u}) \\
\hat{z}^{\prime} & =-\sigma \hat{z}-(\hat{z}-\hat{u})^{2}-\delta \hat{r} \hat{u}+\hat{\epsilon} \tilde{g}(\hat{u}, \hat{r}, \hat{z}, \hat{\epsilon}) \\
\hat{\epsilon}^{\prime} & =-\hat{\epsilon}(\hat{z}-\hat{u}),
\end{aligned}
$$

with

$$
\begin{aligned}
\tilde{f}(\hat{u}, \hat{r}, \hat{\epsilon}) & =\frac{\delta}{1-\hat{r} \hat{\epsilon}}\left(1-\sigma \hat{u}-\frac{\gamma}{\alpha} \hat{u}-\sigma^{2} \hat{r} \hat{u}-\frac{\sigma \gamma}{\alpha} \hat{r} \hat{\epsilon}\right) \quad \text { and } \\
\tilde{g}(\hat{u}, \hat{r}, \hat{z}, \hat{\epsilon}) & =-\hat{\epsilon} \frac{\gamma}{\alpha(\alpha+\hat{\epsilon})}+\left(-\frac{\delta \gamma}{\alpha \sigma} \hat{r}+\frac{\hat{r}}{\sigma} \tilde{f}(\hat{u}, \hat{r}, \hat{\epsilon})+\frac{\gamma}{\alpha \sigma} \hat{z}-\frac{\gamma}{\alpha \sigma} \hat{u}\right) .
\end{aligned}
$$

The curves $l_{j}(j=1,2,3)$ are mapped to

$$
\begin{aligned}
& \hat{l}_{1}=\left\{\left(0, r^{*}, 0,0\right), r^{*} \geq 0\right\} \\
& \hat{l}_{2}=\left\{\left(u^{*}, 0, u^{*}-\frac{\sigma}{2}+\frac{1}{2} \sqrt{\sigma^{2}-4 \sigma u^{*}}, 0\right), u^{*} \leq \frac{\sigma^{2}}{4}\right\}, \quad \text { and } \\
& \hat{l}_{3}=\left\{\left(-\frac{\left(\epsilon^{*}\right)^{2} \gamma}{\alpha\left(\alpha+\epsilon^{*}\right) \sigma}, 0,-\frac{\left(\epsilon^{*}\right)^{2} \gamma}{\alpha\left(\alpha+\epsilon^{*}\right) \sigma}, \epsilon^{*}\right), \epsilon^{*} \geq 0\right\}
\end{aligned}
$$

here, $\hat{l}_{1}$ again corresponds to the unperturbed slow manifold $\mathcal{M}_{V}^{0}$.

We have the following result on the existence of a center manifold for Equation (6.21); see $[3,4,38]$.

Lemma 6.9. There exists $\delta_{\Omega}>0$ such that the origin in Equation (6.21) admits a smooth center manifold $\widehat{W}^{\mathrm{c}}(0)$ that is tangent to the corresponding center eigenspace. The manifold $\widehat{W}^{\mathrm{c}}(0)$ is defined on a neighborhood $\Omega \subset B_{\delta_{\Omega}}(0)$ of the origin, and has the following properties. 
1. The manifold $\widehat{W}^{\mathrm{c}}(0)$ contains the three curves $\hat{l}_{j}(j=1,2,3)$, as well as the continuation of the slow manifold $\mathcal{M}_{V}^{\epsilon}$ and its stable foliation $\mathcal{F}^{\mathrm{s}}\left(\mathcal{M}_{V}^{\epsilon}\right)$. It also includes the center manifold $\mathcal{W}^{\mathrm{c}}(0)$ of the steady state at infinity studied in Lemma 4.2.

2. Within $\Omega, \widehat{W}^{\mathrm{c}}(0)$ can be expressed locally as the graph

$$
\hat{z}=h(\hat{u}, \hat{r}, \hat{\epsilon})=\sum_{|k|>1} h_{k} \hat{u}^{k_{1}} \hat{r}^{k_{2}} \hat{\epsilon}^{k_{3}}
$$

with $k=\left(k_{1}, k_{2}, k_{3}\right)$ and $h(0, \hat{r}, 0)=0$ for any $\hat{r}$. In addition, the leading-order coefficients in the above series are

$$
h_{200}=-\frac{1}{\sigma}, h_{110}=-\frac{\delta}{\sigma}, h_{020}=0, h_{101}=-\frac{\gamma}{\alpha \sigma^{2}}, h_{011}=\frac{\delta(\alpha-\gamma)}{\sigma^{2} \alpha}, \text { and } h_{002}=-\frac{\gamma}{\alpha^{2} \sigma} .
$$

3. There exists a locally invariant unstable foliation of $\Omega=\bigcup_{(\hat{u}, \hat{r}, h(\hat{u}, \hat{r}, \hat{\epsilon}), \hat{\epsilon}) \in \widehat{W}^{\mathrm{c}}(0)} \widehat{M}_{\mathrm{u}}(\hat{u}, \hat{r}, \hat{\epsilon})$, where $\widehat{M}_{\mathrm{u}}(\hat{u}, \hat{r}, \hat{\epsilon})$ is the unstable fiber associated to the base point $(\hat{u}, \hat{r}, \hat{\epsilon})$. The unstable eigenspace is spanned by the vector $e_{z}=\left\{(0,0,1,0)^{T}\right\}$; each unstable fiber can be written as a graph over the unstable eigenspace,

$$
\widehat{M}_{\mathrm{u}}(\hat{u}, \hat{r}, \hat{\epsilon})=\left\{(\hat{u}, \hat{r}, h(\hat{u}, r, \hat{\epsilon}), \hat{\epsilon})+c e_{z}+J(c, \hat{u}, \hat{r}, \hat{\epsilon}) \mid c \in \mathbb{R}\right\},
$$

where $J$ is differentiable and $\|J\|_{C^{1}} \leq K \delta_{\Omega}$ for some $K>0$. Furthermore, $J(0, \hat{u}, \hat{r}, \hat{\epsilon})=0$, as $(\hat{u}, \hat{r}, h(\hat{u}, \hat{r}, \hat{\epsilon}), \hat{\epsilon}) \in \widehat{W}^{\mathrm{c}}(0)$.

Restricting attention to the flow within the center manifold $\widehat{W}^{\mathrm{c}}(0)$, we find that the dynamics of the base points is governed by the following reduced system:

$$
\begin{aligned}
& \hat{u}^{\prime}=-\delta \hat{r} \hat{u}+\hat{\epsilon}\left(-\frac{\delta \gamma}{\alpha \sigma} \hat{r}+\frac{\hat{r}}{\sigma} \tilde{f}(\hat{u}, \hat{r}, \hat{\epsilon})+\frac{\gamma}{\alpha \sigma} h(\hat{u}, \hat{r}, \hat{\epsilon})-\frac{\gamma}{\alpha \sigma} \hat{u}\right) \\
& \hat{r}^{\prime}=-\hat{r} \hat{u}+h(\hat{u}, \hat{r}, \hat{\epsilon}) \hat{r} \\
& \hat{\epsilon}^{\prime}=\hat{\epsilon} \hat{u}-h(\hat{u}, \hat{r}, \hat{\epsilon}) \hat{\epsilon} .
\end{aligned}
$$

The origin remains a non-hyperbolic steady state. The three lines of equilibria $\hat{l}_{j}(j=1,2,3)$ are transformed to the $\hat{r}$-axis, the $\hat{u}$-axis, and a quadratic curve in the plane $\{\hat{r}=0\}$ that is described by the implicit equation $\hat{u}-h(\hat{u}, 0, \hat{\epsilon})=0$, respectively. To understand the dynamics within the center manifold $\widehat{W}^{\mathrm{c}}(0)$, we will utilize geometric desingularization to "blow up" the non-hyperbolic point at the origin in (6.22) to a sphere, whereby hyperbolicity is regained.

To that end, we introduce three charts $\widehat{K}_{1}$ through $\widehat{K}_{3}$, which are defined by the following changes of coordinates:

$$
\begin{array}{ll}
\widehat{K}_{1}: & \hat{u}=\rho_{1} U_{1}, \hat{r}=\rho_{1}, \text { and } \hat{\epsilon}=\rho_{1} E_{1} ; \\
\widehat{K}_{2}: & \hat{u}=\rho_{2}, \hat{r}=\rho_{2} R_{2}, \text { and } \hat{\epsilon}=\rho_{2} E_{2} ; \\
\widehat{K}_{3}: & \hat{u}=\rho_{3} U_{3}, \hat{r}=\rho_{3} R_{3}, \text { and } \hat{\epsilon}=\rho_{3} .
\end{array}
$$

We have the following result.

Lemma 6.10. Let $\kappa>0$, consider the sections $\Delta^{\text {out }}=\{(\hat{u}, \hat{r}, \hat{\epsilon}) \mid \hat{r}=\kappa\}$ and $\Sigma^{\text {in }}=\{(\hat{u}, \hat{r}, \hat{\epsilon}) \mid \hat{\epsilon}=$ $\kappa\}$ in $(\hat{u}, \hat{r}, \hat{\epsilon})$-space, and let $\Pi_{4}: \Delta^{\text {out }} \rightarrow \Sigma^{\text {in }}$ denote the corresponding transition map. Then, for $\epsilon_{0}>0$ sufficiently small, the restriction of the manifold $\mathcal{M}_{V}^{\epsilon} \times\left(0, \epsilon_{0}\right)$ to the center manifold $\widehat{\mathcal{W}}^{\mathrm{c}}(0)$ 
is a two-dimensional manifold that intersects $\Delta^{\text {out }}$ transversely. Similarly, the forward image of $\mathcal{M}_{V}^{\epsilon} \times\left(0, \epsilon_{0}\right)$ under the flow of Equation (6.21) intersects transversely with $\Sigma^{\text {in }}$, where the restriction to that section is described by the graph

$$
\hat{u}=-\frac{\gamma \kappa}{\alpha \sigma}-\sqrt{\epsilon} \kappa \Phi_{3}(\kappa, \epsilon) \quad \text { and } \quad \hat{r}=\frac{\epsilon}{\kappa},
$$

with $\Phi_{3}(\kappa, \epsilon)$ a smooth function of $\kappa$.

The result follows from an analysis of the blown-up vector field that is induced by (6.21) in the three charts $\widehat{K}_{j}$ defined in (6.23), which is relegated to Appendix B.

The tracking of $\mathcal{M}^{+}$is now accomplished by considering first the corresponding base points in the center manifold $\widehat{\mathcal{W}}^{\mathrm{c}}(0)$, where we can apply Lemma 6.10 ; the manifold itself is then tracked by following the unstable fibers associated to these base points:

Lemma 6.11. Let $\epsilon \in\left(0, \epsilon_{0}\right)$, with $\epsilon_{0}>0$ sufficiently small. Within the section $\Sigma^{\text {in }}$, the tracked manifold $\mathcal{M}^{+}$can then be expressed as the graph

$$
\begin{aligned}
& u=-\sqrt{\epsilon} \sigma \hat{\epsilon}_{\mathrm{b}} \Phi\left(\hat{\epsilon}_{\mathrm{b}}\right)+\sigma J^{\hat{u}}\left(c\left(u^{\text {out }}\right), \hat{\epsilon}_{\mathrm{b}}\right)+\sigma R_{\hat{u}}\left(\epsilon, c\left(u^{\text {out }}\right), u^{\text {in }}, u^{\text {out }}\right)+\frac{\gamma}{\alpha} J^{\hat{\epsilon}}\left(c\left(u^{\text {out }}\right), \hat{\epsilon}_{\mathrm{b}}\right) \\
& +\frac{\gamma}{\alpha} R_{\hat{\epsilon}}\left(\epsilon, c\left(u^{\text {out }}\right), u^{\text {in }}, u^{\text {out }}\right) \\
& v=\frac{1}{\kappa} \\
& z=\sigma+c\left(u^{\text {out }}\right)+h\left(-\frac{\gamma \hat{\epsilon}_{\mathrm{b}}}{\alpha \sigma}-\sqrt{\epsilon} \hat{\epsilon}_{\mathrm{b}} \Phi\left(\epsilon, \hat{\epsilon}_{\mathrm{b}}, u^{\text {out }}\right), \frac{\epsilon}{\hat{\epsilon}_{\mathrm{b}}}, \hat{\epsilon}_{\mathrm{b}}\right)+J^{\hat{z}}\left(c\left(u^{\text {out }}\right), \hat{\epsilon}_{\mathrm{b}}\right) \\
& +R_{\hat{z}}\left(\epsilon, c\left(u^{\text {out }}\right), u^{\text {in }}, u^{\text {out }}\right)+\frac{\gamma \hat{\epsilon}_{\mathrm{b}}}{\alpha \sigma}+\sqrt{\epsilon} \hat{\epsilon}_{\mathrm{b}} \Phi\left(\hat{\epsilon}_{\mathrm{b}}\right)-J^{\hat{u}}\left(c\left(u^{\text {out }}\right), \hat{\epsilon}_{\mathrm{b}}\right)-R_{\hat{u}}\left(\epsilon, c\left(u^{\text {out }}\right), u^{\text {in }}, u^{\text {out }}\right) \\
& u^{\text {in }}=u^{\text {in }} \\
& u^{\text {out }}=u^{\text {out }} \text {, }
\end{aligned}
$$

where $c\left(u^{\text {out }}\right)=\mathcal{O}\left(\mathrm{e}^{\frac{C}{\epsilon}}\right)$ for some $C>0$ and where the terms $R_{\hat{x}}(\hat{x}=\hat{u}, \hat{r}, \hat{z}, \hat{\epsilon})$ are exponentially small in $\epsilon$.

Proof. The Exchange Lemma [30, Theorem 3.1] implies that the tracked manifold $\mathcal{M}^{+}$will intersect the section $\Delta^{\text {out }}, C^{1}$ exponentially close in $\epsilon$ to the unstable foliation $\mathcal{F}^{\mathrm{u}}\left(\mathcal{M}_{V}^{\epsilon}\right)$. (We have additional information, in fact, since each submanifold of $\mathcal{M}^{+}$with $u^{\text {in }}$ fixed is $C^{1}$ exponentially close to $\mathcal{F}^{\mathrm{u}}\left(\mathcal{M}_{V}^{\epsilon}\right)$.) To track $\mathcal{M}^{+}$beyond $\Delta^{\text {out }}$, we again transform to the coordinates of chart $K_{2}$, decomposing $\mathcal{M}^{+} \times\left[0, \epsilon_{0}\right)$ into base points within the center manifold $\widehat{\mathcal{W}}^{\mathrm{c}}(0)$ defined in Lemma 6.9 and associated points in the unstable foliation of $\Omega$. (Here, we recall that $\widehat{\mathcal{W}}^{c}(0)$ contains the continuation of the slow manifold $\mathcal{M}_{V}^{\epsilon}$, as well as of its stable foliation $\mathcal{F}^{\mathrm{s}}\left(\mathcal{M}_{V}^{\epsilon}\right)$.)

We track the base points of $\mathcal{M}^{+}$within $\widehat{\mathcal{W}}^{\mathrm{c}}(0)$ first. The analysis of the flow in the center manifold $\widehat{\mathcal{W}}^{\mathrm{c}}(0)$, which requires another blow-up transformation, is given in Appendix $\mathrm{B}$. Here, three charts are employed. In chart $\widehat{K}_{1}$, following desingularization, there exists a two-dimensional center manifold $\mathcal{W}_{1}^{c}(0)$ that represents the continuation of $\mathcal{M}_{V}^{\epsilon}$ in blown-up coordinates; correspondingly, $\mathcal{F}^{\mathrm{s}}\left(\mathcal{M}_{V}^{\epsilon}\right)$ is continued as the stable foliation of that center manifold. We note that the exponential closeness of the tracked manifold $\mathcal{M}^{+}$to the unstable foliation $\mathcal{F}^{\mathrm{u}}\left(\mathcal{M}_{V}^{\epsilon}\right)$ at entry implies that it suffices to track only the center manifold $\mathcal{W}_{1}^{\mathrm{c}}(0)$ in chart $\widehat{K}_{1}$ - all other orbits are exponentially attracted to it. The passage of that manifold past the origin is described in Lemma 6.10, where we obtain an expression for the tracked base points, in analogy to Equation (6.24). In fact, we can 
extend that expression to a neighborhood of the section $\Sigma^{\text {in }}$ : for $|\hat{\epsilon}-\kappa|$ small, the base points can be written as a graph over $\hat{\epsilon}$,

$$
\hat{u}=-\frac{\gamma}{\alpha \sigma} \hat{\epsilon}-\hat{\epsilon} \sqrt{\epsilon} \Phi_{3}(\epsilon, \hat{\epsilon}) \quad \text { and } \quad \hat{r}=\frac{\epsilon}{\hat{\epsilon}},
$$

where $\Phi_{3}(\epsilon, \hat{\epsilon})$ is a smooth function of $\hat{\epsilon}$.

Finally, the manifold $\mathcal{M}^{+}$itself is tracked simply by following the unstable fibers of the center manifold $\mathcal{W}_{1}^{c}(0)$ that are associated to the above base points. Invariance of the family of unstable fibers implies that the tracked manifold will intersect the section $\Sigma^{\text {in }}$ close to the unstable fiber of the associated base point. We also obtain that each submanifold of $\mathcal{M}^{+}$with $u^{\text {in }}$ fixed intersects $\Sigma^{\text {in }} C^{1}$ exponentially close to that fiber.

In sum, we thus have the following expression for $\mathcal{M}^{+}$near its intersection with the section $\Sigma^{\text {in }}$,

$$
\begin{aligned}
& \hat{u}=-\frac{\gamma \hat{\epsilon}_{\mathrm{b}}}{\alpha \sigma}-\sqrt{\epsilon} \hat{\epsilon}_{\mathrm{b}} \Phi\left(\hat{\epsilon}_{\mathrm{b}}\right)+J^{\hat{u}}\left(c\left(u^{\text {out }}\right), \hat{\epsilon}_{\mathrm{b}}\right)+R_{\hat{u}}\left(\epsilon, c\left(u^{\text {out }}\right), u^{\text {in }}, u^{\text {out }}\right) \\
& \hat{r}=\frac{\epsilon}{\hat{\epsilon}_{\mathrm{b}}}+J^{\hat{r}}\left(c\left(u^{\text {out }}\right), \hat{\epsilon}_{\mathrm{b}}\right)+R_{\hat{r}}\left(\epsilon, c\left(u^{\text {out }}\right), u^{\text {in }}, u^{\text {out }}\right) \\
& \hat{z}=h\left(-\frac{\gamma \hat{\epsilon}_{\mathrm{b}}}{\alpha \sigma}-\sqrt{\epsilon} \hat{\epsilon}_{\mathrm{b}} \Phi\left(\epsilon, \hat{\epsilon}_{\mathrm{b}}, u^{\text {out }}\right), \hat{\epsilon}_{\mathrm{b}}, \hat{\epsilon}_{\mathrm{b}}\right)+c\left(u^{\text {out }}\right)+J^{\hat{z}}\left(c\left(u^{\text {out }}\right), \hat{\epsilon}_{\mathrm{b}}\right)+R_{\hat{z}}\left(\epsilon, c\left(u^{\text {out }}\right), u^{\text {in }}, u^{\text {out }}\right) \\
& \hat{\epsilon}=\hat{\epsilon}_{\mathrm{b}}+J^{\hat{\epsilon}}\left(c\left(u^{\text {out }}\right), \hat{\epsilon}_{\mathrm{b}}\right)+R_{\hat{\epsilon}}\left(\epsilon, c\left(u^{\text {out }}\right), u^{\text {in }}, u^{\text {out }}\right),
\end{aligned}
$$

where the terms $R_{\hat{x}}(\hat{x}=\hat{u}, \hat{r}, \hat{z}, \hat{\epsilon})$ are exponentially small in $\epsilon$. Moreover, we have written $J=$ $\left(J^{\hat{u}}, J^{\hat{r}}, J^{\hat{z}}, J^{\hat{\epsilon}}\right)^{T}$ as a function of $c$ and $\hat{\epsilon}_{\mathrm{b}}$, since the base points of interest in $\widehat{\mathcal{W}}^{\mathrm{c}}(0)$ can be given as a graph over $\hat{\epsilon}$. (We remark that the section $\Sigma^{\text {in }}$ can equivalently be defined by $\{\hat{\epsilon}=\kappa\}$ in $(\hat{u}, \hat{r}, \hat{z}, \hat{\epsilon})$-space.) Thus, to restrict our manifold to $\Sigma^{\text {in }}$, we must solve the implicit equation $\hat{\epsilon}_{\mathrm{b}}+J^{\hat{\epsilon}}\left(c, \hat{\epsilon}_{\mathrm{b}}\right)+R_{\hat{\epsilon}}=\kappa$, which has a solution for $\hat{\epsilon}_{\mathrm{b}}=\kappa$ when $(c, \epsilon)=(0,0)$. Applying the Implicit Function Theorem, we find an expression for the base points $\hat{\epsilon}_{\mathrm{b}}(c, \epsilon)$ such that $\mathcal{M}^{+}$intersects $\Sigma^{\text {in }}$. Restricting to the section $\Sigma^{\text {in }}$, we then transform to the rescaled coordinates $(u, v, z)$, recalling that $z=\frac{w}{v}$, to obtain $(6.25)$.

\subsection{The transition map $\Pi_{-1}: \Sigma_{0} \rightarrow \Sigma^{\text {in }}$}

In Sections 6.1 through 6.4, we tracked the evolution of the manifold $\mathcal{M}^{+}$between the sections $\Sigma_{0}$ and $\Sigma^{\text {in }}$; the resulting expression in Equation (6.25) effectively corresponds to an approximation for the composite transition map $\Pi_{4} \circ \Pi_{3} \circ \Pi_{2} \circ \Pi_{1}$. We now track the manifold $\mathcal{M}^{-}$from $\Sigma_{0}$ to $\Sigma^{\text {in }}$ in backward "time" to facilitate a comparison between the two manifolds. The requisite arguments are similar to those presented in Section 6.1, and are hence merely outlined here.

Upon leaving a neighborhood of $\mathcal{M}_{u}$, the manifold $\mathcal{M}^{-}$can be written as the graph

$$
u=u^{\text {in }}, \quad b=\epsilon \widetilde{B}\left(u^{\text {in }}, u^{\text {out }}\right), \quad \text { and } \quad z=\omega .
$$

Lemma 4.2 provides a leading-order approximation for $\mathcal{M}^{-}$within $\Sigma^{\text {in }}$; the exponential closeness of $\mathcal{M}^{-}$to the stable foliation $\mathcal{F}^{\mathrm{s}}\left(\mathcal{M}_{u}\right)$, combined with a finite transition "time", implies that in 
$\Sigma^{\text {in }}$, the manifold $\mathcal{M}^{-}$is described by the graph

$$
\begin{aligned}
u & =u^{\text {in }}+\epsilon \widetilde{U}^{-}\left(u^{\text {in }}, u^{\text {out }}, \epsilon\right) \\
v & =\frac{1}{\kappa} \\
z & =\sigma+H^{\mathrm{s}}\left(u^{\text {in }}\right)+\epsilon \widetilde{Z}^{-}\left(u^{\text {in }}, u^{\text {out }}, \epsilon\right) \\
u^{\text {in }} & =u^{\text {in }} \\
u^{\text {out }} & =u^{\text {out }}
\end{aligned}
$$

in $(u, v, z)$-coordinates.

\subsection{Transverse intersection}

Finally, we show that the extended manifolds $\mathcal{M}^{+} \times \mathcal{I}$ and $\mathcal{M}^{-} \times \mathcal{I}$ intersect transversely in $\Sigma^{\text {in }}$, thus completing the proof of Theorem 1. For $\epsilon=0$, the existence of an intersection can be verified directly. That intersection occurs within the center manifold $\mathcal{W}^{c}(0)$, where $c=0$ and where it is required that $u^{\text {in }}=0$; let $p$ denote the corresponding intersection point.

Consider the tangent spaces of $\mathcal{M}^{+}$and $\mathcal{M}^{-}$at the point $p$. Intuitively, one expects a transverse intersection of the two manifolds, since $\mathcal{M}^{-}$is aligned with the center manifold $\widehat{\mathcal{W}}^{\mathrm{c}}(0)$ in $(\hat{u}, \hat{r}, \hat{z}, \hat{\epsilon})$ coordinates, while $\mathcal{M}^{+}$intersects $\widehat{\mathcal{W}}^{\mathrm{c}}(0)$ transversely. That intuition can be substantiated by calculating the tangent spaces to the manifolds $\mathcal{M}^{+} \times \mathcal{I}$ and $\mathcal{M}^{-} \times \mathcal{I}$ within the section $\Sigma^{\text {in }}$ at their intersection point $p$.

We discuss $\mathcal{M}^{+}$first: given (6.25), a straightforward calculation in $\left(u, z, u^{\text {in }}, u^{\text {out }}\right)$-coordinates yields

$$
T_{p}\left(\mathcal{M}^{+} \times \mathcal{I} \cap \Sigma^{\text {in }}\right)=\operatorname{span}\left\{\left(\begin{array}{c}
\xi_{\mathrm{u}} \\
1+\xi_{z} \\
0 \\
0
\end{array}\right),\left(\begin{array}{l}
0 \\
0 \\
1 \\
0
\end{array}\right)\right\},
$$

where $\xi_{\mathrm{u}}$ and $\xi_{z}$ are shorthand notation for the derivatives of those terms in Equation (6.25) which involve $J$ and $\hat{\epsilon}_{\mathrm{b}}$; we note that these terms are all of the order $\mathcal{O}\left(\delta_{\Omega}\right)$. Here, the structure of the first vector is a result of the dependence of (6.25) on $c$, which in turn encodes its $u^{\text {out }}$-dependence; the second vector is obtained due to $\mathcal{M}^{+}$being independent of $u^{\text {in }}$ to leading order, by Remark 8. Given the exponential expansion of $\mathcal{M}^{+}$near the slow manifold $\mathcal{M}_{V}^{\epsilon}$, the leading-order dependence of $T_{p}\left(\mathcal{M}^{+} \times \mathcal{I} \cap \Sigma^{\text {in }}\right)$ on $u^{\text {out }}$ is exponentially small in $\epsilon$ and hence does not enter the above expressions.

By contrast, the tangent space of $\mathcal{M}^{-}$at the intersection point $p$ is given by

$$
T_{p}\left(\mathcal{M}^{-} \times \mathcal{I} \cap \Sigma^{\text {in }}\right)=\operatorname{span}\left\{\left(\begin{array}{c}
1 \\
\frac{\partial H^{\mathrm{s}}}{\partial u^{\text {in }}}\left(u^{\mathrm{in}}\right) \\
1 \\
0
\end{array}\right),\left(\begin{array}{l}
0 \\
0 \\
0 \\
1
\end{array}\right)\right\} .
$$

The first vector corresponds to the leading-order invariance of $\mathcal{M}^{-}$as a function of $u^{\text {in }}$, while the second vector encodes the $u^{\text {out }}$-independence of that manifold to leading order.

In order for the intersection of the manifolds $\mathcal{M}^{+} \times \mathcal{I}$ and $\mathcal{M}^{-} \times \mathcal{I}$ to be transverse, the vectors $\left(\xi_{\mathrm{u}}, 1+\xi_{z}\right)^{T}$ and $\left(1, \frac{\partial H^{\mathrm{s}}}{\partial u^{\mathrm{in}}}\left(u^{\mathrm{in}}\right)\right)^{T}$ must not be collinear. The components of these vectors depend on the choice of $\kappa$ and $\delta_{\Omega}$. We recall that $H^{\mathrm{s}}\left(u^{\mathrm{in}}\right)$ is defined in Lemma 4.2, where we note that the corresponding derivative remains bounded as $\kappa$ tends to zero. As a consequence, the above 
set of four vectors is linearly independent, which implies that the manifolds $\mathcal{M}^{+} \times \mathcal{I}$ and $\mathcal{M}^{-} \times \mathcal{I}$ intersect transversely. Since these manifolds perturb smoothly as functions of $\phi(\epsilon)=(\ln \epsilon)^{-1}$, the intersection persists for non-zero values of $\epsilon$; by construction, it contains a periodic orbit of Equation (3.5), which concludes the proof of Theorem 1.

\section{Discussion}

In Theorem 1, we have shown the existence of large-amplitude periodic wavetrains in the limit as $\epsilon \rightarrow 0$ in Equation (1.1). We conclude with a discussion of several unresolved, and potentially interesting, questions for future study.

Failure of the condition in Equation (3.8). The proof developed in the present article covers the scenario in which the tracked manifold $\mathcal{M}^{+}$leaves a neighborhood of $\mathcal{M}_{u}$ near its strong unstable foliation. When the condition in (3.8) is not satisfied, we expect orbits to depart the slow manifold $\mathcal{M}_{u}$ along its weak unstable foliation, at $u=\Pi_{0}(0)$. We conjecture that periodic solutions to Equation (1.1) continue to exist due to heteroclinic connections between the weak unstable foliation and the weak unstable point at infinity. It would be interesting to extend the existence proof presented here to that scenario. (Here, we mention [39], where some of the resulting patterned solutions are found using numerical continuation.)

Stability of the constructed patterns. Given the existence of the periodic wavetrains constructed in Theorem 1, a natural question arises as to their stability. Establishing the stability properties of periodic solutions to PDEs is a difficult endeavor in general; however, in our case, there is some hope that the slow-fast structure of Equation (1.1) could be exploited to prove marginal spectral stability. The spectrum of linear operators with periodic coefficients is purely continuous, comprised of loops of spectrum. The corresponding linear eigenvalue problem also has a fast-slow structure, in which case these loops are often concentrated on spectra of the reduced fast problem. Since the latter is planar here, see Equation (3.7), the spectrum of the linearization about heteroclinic fronts could potentially be resolved. On the other hand, the geometric desingularization underlying our approach may introduce complications, making a spectral analysis intractable.

Invasion fronts between mussel-free states and wavetrains. The periodic patterns established here exist in one-parameter families, which are parametrized by the wavespeed. Hence, it is natural to ask which of these patterns will be observed in Equation (1.1) under various assumptions on its initial conditions. A particular case of interest is the selection of a periodic pattern from initial data that is a small perturbation of the mussel-free state at $(u, v)=(1,0)$. Here, any perturbation with non-zero $v$-component will grow and evolve towards some combination of periodic structures. When that perturbation is compactly supported, one can describe the process as the formation of a traveling front connecting the unstable state at $(1,0)$ to a periodic wavetrain. Thus, compactly supported perturbations tend to select a unique patterned solution, which could be analyzed rigorously; see Figure 6 for a numerical simulation of the phenomenon.

To describe the process, one would have to construct traveling front solutions to (1.1) that connect the unstable mussel-free state to a periodic wavetrain. One expects these solutions to exist for all positive wavespeeds, with the selected front being the marginally stable one; see, for example, [37]. Positivity of the front solution would suggest that the selected front should have at least speed $2 \epsilon$, which is - not coincidentally - the linear spreading speed of the unstable state at $(U, V)=(1,0)$ in rescaled coordinates. However, numerical simulation reveals significantly slower invasion speeds. 


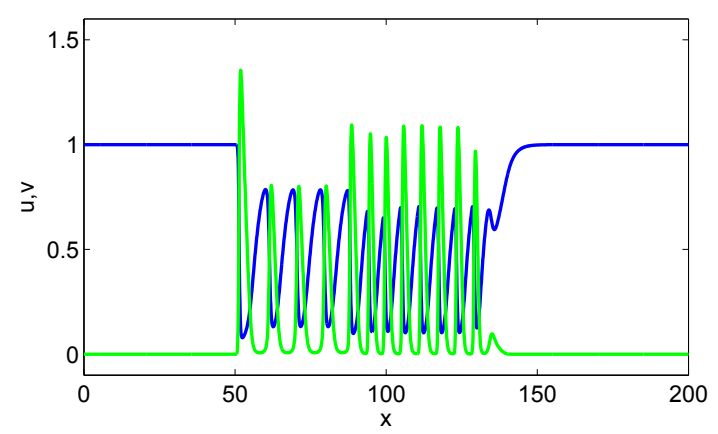

(a) Invasion front.

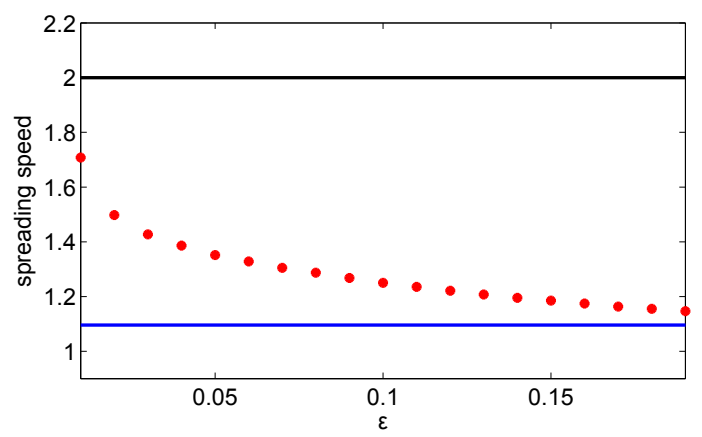

(b) Spreading speed.

Figure 6: A numerical solution of Equation (1.1) with $\alpha=0.4=\delta, \gamma=0.7$, and $\epsilon=0.1$. Initial data was taken to be the mussel-free state throughout, with a small region in the middle of the domain where a non-zero mussel concentration was introduced. The blue and green curves in panel 6(a) represent the $u$-component and a rescaled version of the $v$-component, respectively. In panel 6(b), numerically observed upstream spreading speeds are graphed for the above parameter values, with varying $\epsilon$. The blue line marks the linear spreading speed $\sigma=2 \sqrt{1-\gamma}$ associated to the mussel-free state in the original variables, while the black line at $\sigma=2$ denotes the linear spreading speed in the rescaled variables. Clearly, the observed speed lies between these two extremes.

We hypothesize that the corresponding correction is logarithmic in $\epsilon$, and that it is enforced by a mechanism similar to that in the Fisher-Kolmogorov-Petrowskii-Piscounov equation with cut-off $[2,9]$. The relation to cut-offs can be observed by viewing the transformation of the original PDE to the rescaled coordinates $(U, V, W)$ : the equation governing the mussel concentration is then

$$
V_{t}=\epsilon^{2} V_{x x}+V\left(U-\frac{\epsilon \gamma}{\alpha V+\epsilon}\right)
$$

Linearizing about the state $(U, V)=(1,0)$, one derives the equation $V_{t}=\epsilon^{2} V_{x x}+V(1-\gamma)$, for which the linear spreading speed is $2 \epsilon \sqrt{1-\gamma}$. As soon as $V$ is larger than $\mathcal{O}(\epsilon)$, though, the term multiplying $V$ increases to 1 , suggesting a spreading speed of $2 \epsilon$. The resulting type of cut-off function was considered in [2], yielding a logarithmic correction to the selected wavespeed. One could hope to extend the approach in [9], which is based on GSPT and the blow-up technique, to Equation (1.1). To that end, one would require a more detailed analysis of the passage from unscaled to rescaled coordinates; cf. the proof of Lemma 6.4.

\section{Acknowledgements}

MH is grateful to Anna Ghazaryan and Vahagn Manukian for introducing him to the model studied in the article, and for stimulating discussions. The research of $\mathrm{MH}$ was partially supported by the NSF (DMS-1516155).

\section{A Proof of Lemma 6.1}

In this section, we collect some facts regarding Fenichel-type normal forms and the Exchange Lemma for manifolds with loss-of-stability turning points. For general results, we point the reader to $[26,7,30]$; here, we merely apply these results in the specific context of the slow manifold $\mathcal{M}_{u}$. 


\section{A.1 A Fenichel-type normal form}

We begin by deriving a Fenichel-type normal form for Equation (3.2) that is based on the (generalized) Exchange Lemma; recall Equation (5.1). We have the following result.

Lemma A.1. Let $\sigma<0$, and let $\epsilon \in\left(0, \epsilon_{0}\right)$, with $\epsilon_{0}>0$ sufficiently small. Then, for $u \in$ $\left[-c_{u}, \frac{\sigma^{2}}{4}+c_{u}\right]$, there exists a neighborhood $\mathcal{N}\left(\mathcal{M}_{u}\right)$ of the slow manifold $\mathcal{M}_{u}$ on which a change of coordinates transforms Equation (3.2) to the normal form

$$
\begin{aligned}
b^{\prime} & =B(b, u, z, \epsilon) b \\
u^{\prime} & =\epsilon g(b, u, z, \epsilon) \\
z^{\prime} & =h(b, u, z, \epsilon) z+k(b, u, z, \epsilon) z^{2} .
\end{aligned}
$$

The functions $B(b, u, z, \epsilon), g(b, u, z, \epsilon)$, and $h(b, u, z, \epsilon)$ admit the expansions

$$
\begin{aligned}
g(b, u, z, \epsilon) & =\delta(1-u)+\tilde{g}(u, b, z, \epsilon) \\
B(b, u, z, \epsilon) & =-\frac{\sigma}{2}+\frac{1}{2} \sqrt{\sigma^{2}-4(u-\gamma)}+\widetilde{B}(u, b, z, \epsilon) \\
h(b, u, z, \epsilon) & =-\frac{\sigma}{2}-\frac{1}{2} \sqrt{\sigma^{2}-4(u-\gamma)}+\tilde{h}(u, b, z, \epsilon) ;
\end{aligned}
$$

here, $\tilde{g}, \widetilde{B}$, and $\tilde{h}$ satisfy $\tilde{g}(u, 0,0, \epsilon)=0, \widetilde{B}(u, 0,0,0)=0$, and $\tilde{h}(u, 0,0,0)=0$. Moreover, the strong unstable foliation $\mathcal{F}^{\mathrm{uu}}\left(\mathcal{M}_{u}\right)$ corresponds to the manifold $\{z=0\}$.

Proof: If $\frac{\sigma^{2}}{4}<\gamma$, the manifold $\mathcal{M}_{u}$ remains normally hyperbolic throughout the interval $u \in\left[-c_{u}, \frac{\sigma^{2}}{4}+c_{u}\right]$. Since $\mathcal{M}_{u}$ has one-dimensional stable and unstable foliations in that case, a transformation exists that places the system into the normal form in (A.1); see [11, 17, 16, 18] for details.

We therefore focus on the case where $\frac{\sigma^{2}}{4}>\gamma$. The first step in obtaining the normal form in (A.1) is the identification of a center manifold $\mathcal{W}^{\mathrm{c}}\left(\mathcal{M}_{u}\right)$ for the invariant manifold $\mathcal{M}_{u}$. The existence of $\mathcal{W}^{\mathrm{c}}\left(\mathcal{M}_{u}\right)$ follows from Theorem 1 of [5]. We must ensure that the hypotheses of that theorem are satisfied: the requisite decomposition of the linearization about $\mathcal{M}_{u}$ into unstable and center components is guaranteed, since the dynamics on $\mathcal{M}_{u}$ is of the order $\mathcal{O}(\epsilon)$; therefore, the corresponding linearization is slowly varying. Consequently, there exists a generalized exponential dichotomy whose unstable and center - i.e., weak unstable - projections are $\mathcal{O}(\epsilon)$ perturbations of the associated spectral projections; see, for example, Proposition 6.1 of [6]. The degree of smoothness of the resulting center manifold therefore depends on the relative ratios of the unstable and center eigenvalues of the linearization about $\mathcal{M}_{u}$, which are given by

$$
-\frac{\sigma}{2} \pm \frac{1}{2} \sqrt{\sigma^{2}-4(u-\gamma)}
$$

On the $u$-interval of interest, with $u \in\left[-c_{u}, \frac{\sigma^{2}}{4}+c_{u}\right]$, the unstable and weak unstable eigenvalues always possess a spectral gap, which allows us to apply Theorem 1 of [5], thus obtaining a $C^{1}$ center manifold. Application of the Exchange Lemma will require a higher degree of regularity, however. First, we remark that, if $\left|\frac{\sigma^{2}}{4}-\gamma\right|$ is sufficiently small, the weak unstable eigenvalue is close to zero; hence, the spectral gap will be sufficiently large to yield the requisite smoothness. We will verify in Section A.3 below that the center manifold is, in fact, at least $C^{4}$-smooth in the $(\sigma, \gamma)$-regime that is of interest to us; for the time being, we will proceed assuming at least $C^{4}$-smoothness.

Under that assumption, coordinates can then be chosen near $\mathcal{M}_{u}$ such that Equation (3.2) is transformed into (A.1), as claimed; the requisite change of coordinates is constructed in Lemma 
3.1 of [26]. Let $b$ correspond to the unstable direction in (A.1), and let $(u, z)$ represent the center directions. Then, the functions $B$ and $h$ in (A.1) satisfy $B(0, b, u, 0)>0$ and $h(u, 0, z)<0$ $(h(u, 0, z)>0)$ for $u<\gamma(u>\gamma)$; in fact, we have the expansions in (A.2) for $g, B$, and $h$, as claimed.

\section{A.2 The condition in Equation (3.8)}

We intend to apply the generalized Exchange Lemma, developed in [26, 7, 30], which will allow us to track manifolds that approach the slow manifold $\mathcal{M}_{u}$ near its stable foliation, and which subsequently depart near its strong unstable foliation. Specifically, we are interested in manifolds which enter a neighborhood of $\mathcal{M}_{u}$ near $u=0$, and which then depart at $u=\frac{\sigma^{2}}{4}$. In the process, we will derive the condition in (3.8), which ensures that the tracked manifold is not repelled away prematurely from $\mathcal{M}_{u}$.

Since $u$ is one-dimensional and since $g(b, u, z, \epsilon) \neq 0$ in a neighborhood of $\mathcal{M}_{u}$ for $0 \leq u<1$, the independent variable may be rescaled so that (A.1) becomes

$$
\begin{aligned}
& \frac{d b}{d \tau}=\mu_{+}(u) b+\widetilde{B}(u, b, z, \epsilon) b \\
& \frac{d u}{d \tau}=\epsilon \\
& \frac{d z}{d \tau}=\mu_{-}(u) z+\widetilde{Z}(u, b, z, \epsilon) z^{2}
\end{aligned}
$$

where $\mu_{ \pm}(u)=\frac{-\sigma \pm \sqrt{\sigma^{2}-4(u-\gamma)}}{2 \delta(1-u)}$. Suppose that an orbit of Equation (A.1) enters a neighborhood of $\mathcal{M}_{u}$ near the point $(b, u, z)=\left(0, u^{\text {in }}, \kappa\right)$, and let $I^{-}=[0, \gamma]$ and $I^{+}=\left[\gamma, \frac{\sigma^{2}}{4}+\gamma\right]$. Define the corresponding Poincaré map $\Pi_{0}: I^{-} \rightarrow I^{+}$as follows: given (A.3), note that the leading-order behavior of $z$ and $u$ is related through the differential equation

$$
\frac{d z}{d u}=\frac{-\sigma-\sqrt{\sigma^{2}-4(u-\gamma)}}{2 \epsilon \delta(1-u)} .
$$

We integrate the above equation from $u=u^{\text {in }} \in I^{-}$to $u=u^{\text {out }} \in I^{+}$, noting that the integrand remains real on that interval. (The following argument can be extended to the case where $u^{\text {out }}>$ $\frac{\sigma^{2}}{4}+\gamma$; however, since we are only interested in the dynamics of (A.3) for $u<\frac{\sigma^{2}}{4}+c_{u}$, we do not consider that case here.) Define $\Pi_{0}\left(u^{\text {in }}\right)=u^{\text {out }}$ as the smallest value strictly larger than $u^{\text {in }}$ for which

$$
\int_{u^{\text {in }}}^{u^{\text {out }}} \frac{-\sigma-\sqrt{\sigma^{2}-4(u-\gamma)}}{1-u} d u=0 .
$$

For each $\sigma<0$, we then set $u^{\text {in }}=0$, and we require that $\Pi_{0}(0)>\frac{\sigma^{2}}{4}$, i.e., that

$$
\int_{0}^{\frac{\sigma^{2}}{4}} \frac{-\sigma-\sqrt{\sigma^{2}-4(u-\gamma)}}{1-u} d u<0,
$$

which gives the condition in (3.8).

Remark 9. We note another property of the map $\Pi_{0}$ : since the quantity $-\frac{\sigma}{2}-\frac{1}{2} \sqrt{\sigma^{2}-4(u-\gamma)}$ is decreasing as a function of $u$, we have $\frac{d \Pi_{0}}{d u} \leq 0$. 


\section{A.3 The Exchange Lemma}

The Exchange Lemma requires the terms in the normal form in (A.1) to be at least $C^{2}$-smooth in their arguments. To ensure that requirement, we will show that the center manifold $\mathcal{W}^{\mathrm{c}}\left(\mathcal{M}_{u}\right)$ corresponding to $\mathcal{M}_{u}$ can be constructed to be $C^{4}$-smooth, which guarantees a $C^{3}$ normal form.

To determine the degree of smoothness of $\mathcal{W}^{\mathrm{c}}\left(\mathcal{M}_{u}\right)$, we must resolve the spectral gap between the unstable and weak unstable eigenvalues in (A.2). We seek integer values of $r$ such that

$$
-\frac{\sigma}{2}+\frac{1}{2} \sqrt{\sigma^{2}-4(u-\gamma)}>r\left[-\frac{\sigma}{2}-\frac{1}{2} \sqrt{\sigma^{2}-4(u-\gamma)}\right]
$$

for all $u \in\left[-c_{u}, \frac{\sigma^{2}}{4}+c_{u}\right]$. Due to the monotonicity of these eigenvalues, the above inequality will be satisfied everywhere on that interval if it is satisfied at $u=\frac{\sigma^{2}}{4}+c_{u}$. We therefore seek $r$ such that

$$
-\frac{\sigma}{2}+\sqrt{\gamma-c_{u}}>r\left(-\frac{\sigma}{2}-\sqrt{\gamma-c_{u}}\right)
$$

or, equivalently,

$$
\frac{r+1}{r-1} \sqrt{\gamma-c_{u}}>-\frac{\sigma}{2}
$$

Determining the values of $r$ for which (A.4) holds requires untangling the relationship between $\sigma$ and $\gamma$. Suppose first that $\gamma<\frac{1}{2}$; one can then verify that

$$
\int_{0}^{\gamma} \frac{-\sigma-\sqrt{\sigma^{2}-4(u-\gamma)}}{1-u} d u+\int_{\gamma}^{2 \gamma} \frac{-\sigma-\sqrt{\sigma^{2}-4(u-\gamma)}}{1-u} d u>0
$$

where we have split the interval of integration to emphasize that the first integral is negative, while the second one is positive. Therefore, when $\gamma<\frac{1}{2}$, we have $\Pi_{0}(0)<2 \gamma$, which implies $\frac{\sigma^{2}}{4}<\Pi_{0}(0)<2 \gamma$ by the condition in (3.8). Hence, (A.4) reduces to

$$
\frac{r+1}{r-1}>\frac{\sqrt{2 \gamma}}{\sqrt{\gamma-4 c_{u}}}
$$

in that case, which can be satisfied for $r=4$, with $c_{u}$ sufficiently small. For $\gamma>\frac{1}{2}$, we instead use the condition that $\frac{\sigma^{2}}{4}<1$, whence (A.4) reduces to

$$
\sqrt{\gamma-c_{u}}>\frac{r-1}{r+1}
$$

Since that relation can again be satisfied with $r=4$, for any $\gamma>\frac{1}{2}$ and $c_{u}$ sufficiently small, we conclude that the center manifold $\mathcal{W}^{\mathrm{c}}\left(\mathcal{M}_{u}\right)$ constructed in the proof of Lemma A.1 is $C^{4}$-smooth on the $u$-interval of interest and, hence, that the Fenichel-type normal form in (A.1) is at least $C^{2}$.

We now apply the Exchange Lemma. The formulation of the generalized Exchange Lemma see Theorem 2.4 of [30], for example - applies to manifolds that intersect transversely with the stable foliation of the corresponding slow manifold on entry. The Exchange Lemma then gives the configuration of that manifold, and of its tangent space, as it exits a neighborhood of the slow manifold. That is not the case here, as we have a patch of initial conditions $\left(\mathcal{M}_{0}\right)$ that enter and exit a neighborhood of the slow manifold near specified points; thus, no transversality is assumed, and we require a modified version of the Exchange Lemma, analogous to the one presented in [35], to address the lack of transversality. However, the modified version of the Exchange Lemma in [35] does not encompass the generalized version that is applicable to loss-of-stability turning points. Hence, we reconcile these two versions here in the context of Equation (A.3). 
Recall the boundary value problem in (5.1), with boundary conditions as in (5.4), and consider the two-dimensional submanifold of $\mathcal{M}^{+}$with $u^{\text {in }}$ fixed, which was denoted by $\mathcal{K}_{u^{\text {in }}}$ in the statement of Lemma 6.1. Owing to the condition in (3.8), the manifold $\mathcal{K}_{u^{\text {in }}}$ exits a neighborhood of $\mathcal{M}_{u}$ $C^{0}$ exponentially close to its strong unstable foliation. The primary goal of the modified Exchange Lemma is to obtain $C^{1}$ closeness, which is often accomplished using differential forms. Let $\delta b, \delta u$ and $\delta z$ denote differential forms that project vectors onto the coordinate axes in Equation (5.1); then, differential equations describing the evolution of these projections are given by the variational equation about the orbit $(b, u, z)$. Consideration of one-forms is typically insufficient to track tangent planes, as most tangent vectors will align with the strong unstable direction in the system. Thus, we define the differential two-forms $P_{b u}=\delta b \wedge \delta u, P_{b z}=\delta b \wedge \delta z$, and $P_{u z}=\delta u \wedge \delta z$. These twoforms yield the areas of the parallelograms projected onto various coordinate planes; for example, $P_{b z}$ gives the area of the parallelogram formed by two vectors projected onto the $(b, z)$-coordinate plane [17]. As before, differential equations can be obtained that describe how the corresponding projected areas evolve along the orbit $(b, u, z)$; these equations are non-autonomous and, to leading order, diagonal:

$$
P_{b u}^{\prime}=\mu_{+}(u) P_{b u}+\ldots, \quad P_{b z}^{\prime}=\left[\mu_{+}(u)+\mu_{-}(u)\right] P_{b z}+\ldots, \quad \text { and } \quad P_{u z}^{\prime}=\mu_{-}(u) P_{u z}+\ldots
$$

Owing again to the condition in (3.8), direct integration shows that the $P_{b u}$-component dominates to leading order.

Two issues remain: firstly, we must consider higher-order terms in (A.5) to verify that the above leading-order dynamics dominates; secondly, since we do not have transversality, we require that the tracked manifold $\mathcal{K}_{u^{\text {in }}}$ has non-zero projection onto the unstable foliation of $\mathcal{M}_{u}$ within the section $\{z=\omega\}$. For a treatment of the first issue, we refer the reader to the proof of Theorem 4.4 in [26], where the $C^{2}$-smoothness of the normal form in (A.1) can be used to control higher-order terms. Due to the three-dimensional nature of our problem, a direct calculation reveals that the leading-order solution for $P_{b u}$ dominates in (A.5) unless $P_{b u}$ vanishes at $\tau=0$. However, since we have restricted to $u=u^{\text {in }}$ and $z=\omega$, we are left with only one degree of freedom, and uniqueness of solutions implies that $P_{b u}(0) \neq 0$. Therefore, we have established Lemma 6.1.

\section{B Proof of Lemma 6.10}

In this section, we present the proof of Lemma 6.10. We first analyze separately the dynamics of the blown-up vector field induced by Equation (6.22) in each of the three coordinate charts $\widehat{K}_{j}$ $(j=1,2,3)$ defined in (6.23). Subsequently, we combine our results into an approximation for the transition map $\Pi_{4}$.

\section{B.1 Dynamics in chart $\widehat{K}_{1}$}

In chart $\widehat{K}_{1}$, we employ the coordinates

$$
\hat{u}=\rho_{1} U_{1}, \hat{r}=\rho_{1}, \text { and } \hat{\epsilon}=\rho_{1} E_{1} ;
$$

in these coordinates, Equation (6.22) transforms to

$$
\begin{aligned}
U_{1}^{\prime}= & -\delta \rho_{1} U_{1}+\rho_{1} U_{1}^{2}-U_{1} h\left(\rho_{1} U_{1}, \rho_{1}, \rho_{1} E_{1}\right) \\
& \quad+E_{1}\left(-\frac{\delta \gamma}{\alpha \sigma} \rho_{1}+\frac{\rho_{1}}{\sigma} \tilde{f}\left(\rho_{1} U_{1}, \rho_{1}, \rho_{1} E_{1}\right)+\frac{\gamma}{\alpha \sigma} h\left(\rho_{1} U_{1}, \rho_{1}, \rho_{1} E_{1}\right)-\frac{\gamma}{\alpha \sigma} \rho_{1} U_{1}\right) \\
\rho_{1}^{\prime}= & -\rho_{1}^{2} U_{1}+\rho_{1} h\left(\rho_{1} U_{1}, \rho_{1}, \rho_{1} E_{1}\right) \\
E_{1}^{\prime}= & 2 \rho_{1} U_{1} E_{1}-2 E_{1} h\left(\rho_{1} U_{1}, \rho_{1}, \rho_{1} E_{1}\right) .
\end{aligned}
$$


Since $h$ contains terms of second order or above, we write $h\left(\rho_{1} U_{1}, \rho_{1}, \rho_{1} E_{1}\right)=\rho_{1}^{2} H_{1}\left(U_{1}, \rho_{1}, E_{1}\right)$. Also, since the entire vector field in (B.2) is multiplied by a factor of $\rho_{1}$, we rescale the independent variable to remove that factor; thus, we arrive at the system

$$
\begin{aligned}
U_{1}^{\prime}= & -\delta U_{1}+U_{1}^{2}-\rho_{1} U_{1} H_{1}\left(U_{1}, \rho_{1}, E_{1}\right) \\
& \quad+E_{1}\left(-\frac{\delta \gamma}{\alpha \sigma}+\frac{1}{\sigma} \tilde{f}\left(\rho_{1} U_{1}, \rho_{1}, \rho_{1} E_{1}\right)+\frac{\gamma}{\alpha \sigma} \rho_{1} H_{1}\left(U_{1}, \rho_{1}, E_{1}\right)-\frac{\gamma}{\alpha \sigma} U_{1}\right) \\
\rho_{1}^{\prime}= & -\rho_{1} U_{1}+\rho_{1}^{2} H_{1}\left(U_{1}, \rho_{1}, E_{1}\right) \\
E_{1}^{\prime}= & 2 E_{1} U_{1}-2 \rho_{1} E_{1} H_{1}\left(U_{1}, \rho_{1}, E_{1}\right) .
\end{aligned}
$$

As $H_{1}\left(0, \rho_{1}, 0\right)=0$, the Equation (B.3) has a line of equilibria at $\left(U_{1}, \rho_{1}, E_{1}\right)=\left(0, \rho^{*}, 0\right)$. Each equilibrium has two zero eigenvalues and a single negative eigenvalue. The center eigenspace of the steady state at $\left(0, \rho^{*}, 0\right)$ is spanned by the vectors $(0,1,0)^{T}$ and $\left(1,0, \frac{\alpha \sigma}{\alpha-\gamma}\right)^{T}$.

Restricting to the surface of the blow-up sphere, i.e., to $\left\{\rho_{1}=0\right\}$, Equation (B.3) reduces to

$$
\begin{aligned}
U_{1}^{\prime} & =-\delta U_{1}+U_{1}^{2}+E_{1}\left[\frac{\delta(\alpha-\gamma)}{\alpha \sigma}-\frac{\gamma}{\alpha \sigma} U_{1}\right] \\
E_{1}^{\prime} & =2 U_{1} E_{1},
\end{aligned}
$$

due to $\tilde{f}(0,0,0)=\frac{1}{\delta}$. The origin is a steady state with one zero eigenvalue and one negative eigenvalue; the stable eigendirection corresponds to the invariant line $\left\{E_{1}=0\right\}$. There exists a center manifold $\mathcal{W}_{1}^{\mathrm{c}}(0)$ for the singular problem that can be written as a smooth graph over $E_{1}$, with expansion

$$
U_{1}=\frac{\alpha-\gamma}{\alpha \sigma} E_{1}+\mathcal{O}\left(E_{1}^{2}\right)
$$

The reduced dynamics within $\mathcal{W}_{1}^{\mathrm{c}}(0)$ is described by the scalar equation

$$
E_{1}^{\prime}=2 \frac{\alpha-\gamma}{\alpha \sigma} E_{1}^{2}+\mathcal{O}\left(E_{1}^{3}\right)
$$

Since $\gamma>\alpha$ in the parameter regime of interest, and since $\sigma<0$, it follows that any orbit with positive initial $E_{1}$-value will diverge from the origin.

The center manifold for the full system, Equation (B.3), can be represented as a graph $U_{1}=$ $G_{1}\left(\rho_{1}, E_{1}\right)$. Note that $G_{1}\left(\rho_{1}, 0\right)=0$, since $\mathcal{W}_{1}^{c}(0)$ contains the line of equilibria along the $\rho_{1}$-axis. The reduced dynamics in that center manifold is given by

$$
\begin{aligned}
\rho_{1}^{\prime} & =-\rho_{1}\left(G_{1}-\rho_{1} H_{1}\right) \\
E_{1}^{\prime} & =2 E_{1}\left(G_{1}-\rho_{1} H_{1}\right),
\end{aligned}
$$

which can be linearized by dividing out the common factor $G_{1}-\rho_{1} H_{1}$ :

$$
\begin{aligned}
\rho_{1}^{\prime} & =-\rho_{1} \\
E_{1}^{\prime} & =2 E_{1} .
\end{aligned}
$$

Clearly, orbits that are initialized off the center manifold $\mathcal{W}_{1}^{c}(0)$ converge to it exponentially.

\section{B.2 Dynamics in chart $\widehat{K}_{2}$}

The coordinates in chart $\widehat{K}_{2}$ are defined by

$$
\hat{u}=\rho_{2}, \quad \hat{r}=\rho_{2} R_{2}, \quad \text { and } \quad \hat{\epsilon}=\rho_{2} E_{2},
$$


whereby Equation (6.22) is transformed to

$$
\begin{aligned}
\rho_{2}^{\prime}= & -\delta \rho_{2} R_{2}+E_{2}\left(-\frac{\delta \gamma}{\alpha \sigma} \rho_{2} R_{2}+\frac{1}{\sigma} \rho_{2} R_{2} \tilde{f}\left(\rho_{2}, \rho_{2} R_{2}, \rho_{2} E_{2}\right)+\frac{\gamma}{\alpha \sigma} \rho_{2}^{2} H_{2}\left(\rho_{2}, \rho_{2} R_{2}, \rho_{2} E_{2}\right)-\frac{\gamma}{\alpha \sigma} \rho_{2}\right) \\
R_{2}^{\prime}= & -R_{2}+\rho_{2} H_{2}\left(\rho_{2}, R_{2}, E_{2}\right) R_{2}+\delta R_{2}^{2} \\
& \quad-R_{2} E_{2}\left(-\frac{\delta \gamma}{\alpha \sigma} R_{2}+\frac{1}{\sigma} R_{2} \tilde{f}\left(\rho_{2}, \rho_{2} R_{2}, \rho_{2} E_{2}\right)+\frac{\gamma}{\alpha \sigma} \rho_{2} H_{2}\left(\rho_{2}, \rho_{2} R_{2}, \rho_{2} E_{2}\right)-\frac{\gamma}{\alpha \sigma}\right) \\
E_{2}^{\prime}= & E_{2}+\delta R_{2} E_{2}-\rho_{2} E_{2} H_{2}\left(\rho_{2}, R_{2}, E_{2}\right) \\
& \quad-E_{2}^{2}\left(-\frac{\delta \gamma}{\alpha \sigma} R_{2}+\frac{1}{\sigma} R_{2} \tilde{f}\left(\rho_{2}, \rho_{2} R_{2}, \rho_{2} E_{2}\right)+\frac{\gamma}{\alpha \sigma} \rho_{2} H_{2}\left(\rho_{2}, \rho_{2} R_{2}, \rho_{2} E_{2}\right)-\frac{\gamma}{\alpha \sigma}\right),
\end{aligned}
$$

following a rescaling of the resulting vector field by a factor of $\rho_{2}$; here, we have again written $h\left(\rho_{2}, \rho_{2} R_{2}, \rho_{2} E_{2}\right)=\rho_{2}^{2} H_{2}\left(\rho_{2}, R_{2}, E_{2}\right)$.

As before, we only need to consider the singular flow on the surface of the sphere, i.e., for $\rho_{2}=0$; there, we have the dynamics

$$
\begin{aligned}
& R_{2}^{\prime}=-R_{2}+\delta R_{2}^{2}-R_{2} E_{2}\left[\frac{\delta(\alpha-\gamma)}{\alpha \sigma} R_{2}-\frac{\gamma}{\alpha \sigma}\right] \\
& E_{2}^{\prime}=E_{2}+\delta R_{2} E_{2}-E_{2}^{2}\left[\frac{\delta(\alpha-\gamma)}{\alpha \sigma} R_{2}-\frac{\gamma}{\alpha \sigma}\right] .
\end{aligned}
$$

The steady state at the origin is a saddle; the corresponding stable and unstable eigendirections align with the invariant equator $\left\{R_{2}=0\right\}$ of the blow-up sphere and the invariant line $\left\{E_{2}=0\right\}$, respectively.

\section{B.3 Dynamics in chart $\widehat{K}_{3}$}

We proceed to chart $\widehat{K}_{3}$, wherein

$$
\hat{u}=\rho_{3} U_{3}, \hat{r}=\rho_{3} R_{3}, \text { and } \hat{\epsilon}=\rho_{3} .
$$

In these coordinates, we find after desingularization

$$
\begin{aligned}
U_{3}^{\prime}= & -\delta U_{3} R_{3}-U_{3}^{2}-\frac{\delta \gamma}{\alpha \sigma} R_{3}+\frac{1}{\sigma} R_{3} \tilde{f}\left(\rho_{3} U_{3}, \rho_{3} R_{3}, \rho_{3}\right)+\frac{\gamma}{\alpha \sigma} \rho_{3} H_{3}\left(U_{3}, R_{3}, \rho_{3}\right)-\frac{\gamma}{\alpha \sigma} U_{3} \\
& \quad+U_{3} \rho_{3} H_{3}\left(U_{3}, R_{3}, \rho_{3}\right) \\
R_{3}^{\prime}= & -2 U_{3} R_{3}+2 R_{3} \rho_{3} H_{3}\left(U_{3}, R_{3}, \rho_{3}\right) \\
\rho_{3}^{\prime}= & U_{3} \rho_{3}-\rho_{3}^{2} H_{3}\left(U_{3}, R_{3}, \rho_{3}\right) .
\end{aligned}
$$

Restricted to the surface of the sphere, the system in (B.8) becomes

$$
\begin{aligned}
U_{3}^{\prime} & =-\delta U_{3} R_{3}-U_{3}^{2}+\frac{\delta(\alpha-\gamma)}{\alpha \sigma} R_{3}-\frac{\gamma}{\alpha \sigma} U_{3} \\
R_{3}^{\prime} & =-2 U_{3} R_{3} .
\end{aligned}
$$

The above singular system has a semi-hyperbolic equilibrium at the origin and a stable steady state at $\left(U_{3}, R_{3}\right)=\left(-\frac{\gamma}{\alpha \sigma}, 0\right)$. The rectangular strip defined by $R_{3}>0$ and $U_{3} \in\left[0,-\frac{\gamma}{\alpha \sigma}\right]$ constitutes a trapping region: all orbits within that rectangle - aside from the origin - are attracted to the 
stable state at $\left(-\frac{\gamma}{\alpha \sigma}, 0\right)$, which they approach along the weak unstable eigendirection $\left\{R_{3}=0\right\}$. For future reference, we remark that, in $\left(U_{1}, E_{1}\right)$-coordinates, that trapping region transforms to

$$
E_{1}>0 \quad \text { and } \quad U_{1} \in\left[0,-\frac{\gamma}{\alpha \sigma} E_{1}\right] .
$$

The vector field in (B.8) can be desingularized after division by the (non-zero) quantity $U_{3}-$ $\rho_{3} H_{3}$. As a result, the dynamics in $R_{3}$ and $\rho_{3}$ is linearized,

$$
\begin{aligned}
U_{3}^{\prime} & =-U_{3}-\frac{\gamma}{\alpha \sigma}+R_{3} G_{3}\left(U_{3}, R_{3}, \rho_{3}\right) \\
R_{3}^{\prime} & =-2 R_{3} \\
\rho_{3}^{\prime} & =\rho_{3},
\end{aligned}
$$

where

$$
G_{3}\left(U_{3}, R_{3}, \rho_{3}\right)=\frac{-\delta U_{3}-\frac{\delta \gamma}{\alpha \sigma}+\frac{1}{\sigma} \tilde{f}\left(\rho_{3} U_{3}, \rho_{3} R_{3}, \rho_{3}\right)}{U_{3}-\rho_{3} H_{3}\left(U_{3}, R_{3}, \rho_{3}\right)} .
$$

The equation for $U_{3}$ cannot necessarily be linearized due to the presence of resonances between the eigenvalues of (B.10); see also [21] for a related discussion. We will be interested in orbits that approach $\left(-\frac{\gamma}{\alpha \sigma}, 0\right)$ near its weak unstable eigendirection, and that then depart along the unstable manifold. That passage is naturally studied in Shil'nikov variables: there exists a smooth change of coordinates that transforms (B.10) to

$$
\begin{aligned}
\widetilde{U}_{3}^{\prime} & =-\widetilde{U}_{3}+\rho_{3} R_{3} \widetilde{G}_{3}\left(\widetilde{U}_{3}, R_{3}, \rho_{3}\right) \\
R_{3}^{\prime} & =-2 R_{3} \\
\rho_{3}^{\prime} & =\rho_{3} ;
\end{aligned}
$$

see for example [14]. (Here, the point $\left(-\frac{\gamma}{\alpha \sigma}, 0\right)$ has additionally been shifted to the origin.) Consider orbits which enter a neighborhood of the origin in the section

$$
\widetilde{\Sigma}_{3}^{\text {in }}=\left\{\left(\widetilde{U}_{3}, R_{3}, \rho_{3}\right) \mid \widetilde{U}_{3}=-\kappa\right\}
$$

for some $\kappa>0$, and which depart through the section

$$
\Sigma_{3}^{\text {out }}=\left\{\left(\widetilde{U}_{3}, R_{3}, \rho_{3}\right) \mid \rho_{3}=\kappa\right\}
$$

in the coordinates of chart $\widehat{K}_{3}$. Making use of the Shil'nikov normal form in (B.11), one can prove the existence of such orbits for all transition "times" $\widetilde{Z} \gg 1$. Furthermore, rigorous asymptotic expansions can be derived for the corresponding trajectory in the exit section; in particular, there exists some $\omega>0$ such that

$$
\widetilde{U}_{3}=-\kappa \mathrm{e}^{-\widetilde{Z}}+\mathcal{O}\left(\mathrm{e}^{-\widetilde{Z}-\omega}\right)
$$

in $\Sigma^{\text {in }}$.

\section{B.4 The transition map $\Pi_{4}: \Delta^{\text {out }} \rightarrow \Sigma^{\text {in }}$}

In this subsection, we combine the analysis in the individual charts to describe the transition map $\Pi_{4}$; we only sketch the argument here, omitting much of the detail.

We first consider the dynamics on the surface of the blow-up sphere. Note that the positive octant thereon is invariant due to the invariance of the $U_{1}$-axis in chart $\widehat{K}_{1}$ (or, equivalently, of the $R_{2}$-axis), the invariance of the $E_{2}$-axis in chart $\widehat{K}_{2}$ (or, equivalently, of the $U_{3}$-axis), and the 
fact that the vector field on the $E_{1}$-axis always points in the positive direction. The steady state at $\left(U_{3}, R_{3}\right)=\left(-\frac{\gamma}{\alpha \sigma}, 0\right)$ in chart $\widehat{K}_{3}$ attracts all strictly positive orbits. Comparing the expansion for the center manifold $\mathcal{W}_{1}^{\mathrm{c}}(0)$ in chart $\widehat{K}_{1}$, see (B.4), with the trapping region defined in (B.9), we find that orbits departing $\mathcal{W}_{1}^{\mathrm{c}}(0)$ in $\widehat{K}_{1}$ will also approach the stable steady state in $\widehat{K}_{3}$ along the $R_{3}$-axis; see Figure 7 .

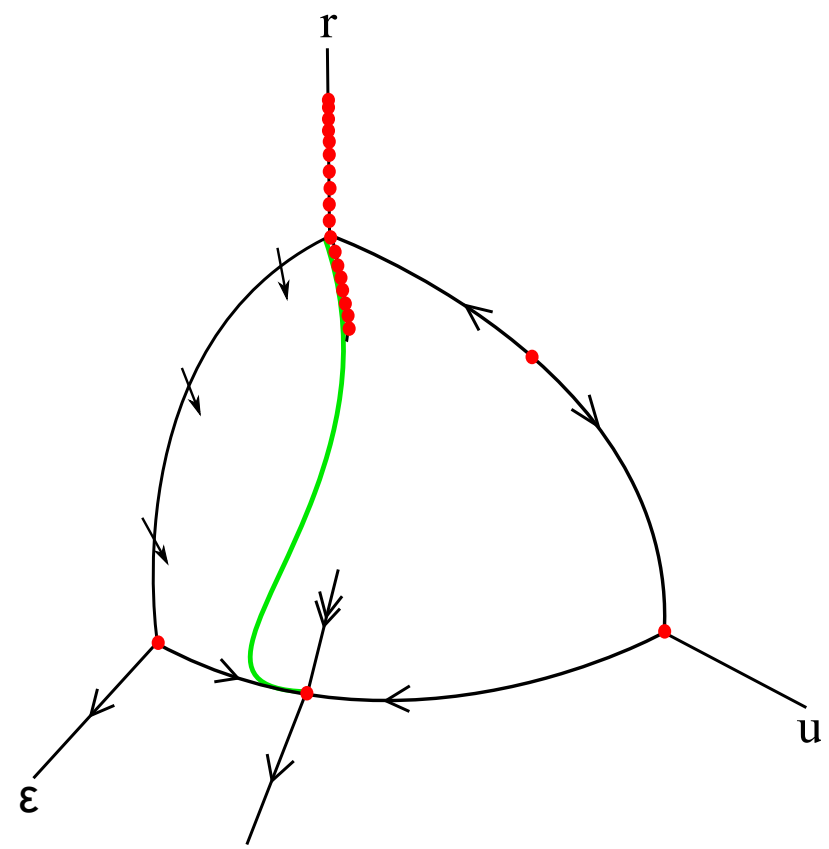

Figure 7: The blown-up vector field induced by Equation (6.22) near the origin. Note the invariance of the positive octant of the desingularized sphere and the existence of a singular connection, shown in green, between the north pole of the blow-up sphere and the steady state at its equator.

Expanding the unstable foliation $\mathcal{F}^{\mathrm{u}}\left(\mathcal{M}_{V}^{\epsilon}\right) \times\left[0, \epsilon_{0}\right)$ and transforming to $(u, r, z, \epsilon)$-coordinates, we find a transverse intersection with the center manifold $\mathcal{W}^{\mathrm{c}}(0)$.

Remark 10. We remark that Fenichel foliation is not, in general, equivalent to the foliation of a center manifold. Hence, in theory, we must track a number of base points and their corresponding unstable fibers. In reality, however, the exponential contraction in chart $\widehat{K}_{1}$ implies that we may focus on representative base points and their unstable fibers, as all other points will be mapped exponentially close to the former in finite time.

We proceed by considering the continuation of the slow manifold $\mathcal{M}_{V}^{\epsilon}$ within the center manifold $\mathcal{W}^{\mathrm{c}}(0)$. We begin by transforming the section $\Delta^{\text {out }}$ to chart $\widehat{K}_{1}$,

$$
\Delta_{1}^{\text {out }}=\left\{\left(U_{1}, \rho_{1}, E_{1}\right) \mid \rho_{1}=\kappa\right\},
$$

for some $\kappa>0$; the manifold $\mathcal{M}_{V}^{\epsilon}$ intersects $\Delta_{1}^{\text {out }}$, and is described by the graph

$$
U_{1}=G_{1}\left(\kappa, \frac{\epsilon}{\kappa^{2}}\right) \quad \text { and } \quad E_{1}=\frac{\epsilon}{\kappa^{2}}
$$

therein. After desingularization, the dynamics within $\mathcal{W}^{\mathrm{c}}(0)$ is linearized, and given as in Equation (B.5). Using the linearized dynamics, we find that within the section

$$
\widehat{\Sigma}_{1}^{\text {out }}=\left\{\left(U_{1}, \rho_{1}, E_{1}\right) \mid E_{1}=\kappa\right\},
$$


the continuation of the slow manifold $\mathcal{M}_{V}^{\epsilon}$ can be expressed as the graph

$$
U_{1}=G_{1}\left(\kappa, \sqrt{\frac{\epsilon}{\kappa}}\right) \text { and } \rho_{1}=\sqrt{\frac{\epsilon}{\kappa}} .
$$

Now, we track that manifold as it evolves near the surface of the sphere to the intersection with the section

$$
\widehat{\Sigma}_{3}^{\text {in }}=\left\{\left(U_{3}, R_{3}, \rho_{3}\right) \mid U_{3}=-\frac{\gamma}{\sigma \alpha}-\kappa\right\},
$$

in the coordinates of chart $\widehat{K}_{3}$. Since the transition "time" between the sections $\Delta_{1}^{\text {out }}$ and $\widehat{\Sigma}^{\text {in }}$ is finite and independent of $\epsilon$, we find that the manifold $\mathcal{M}_{V}^{\epsilon}$ is described by the graph

$$
R_{3}=\kappa^{2} \Psi_{3}(\kappa, \epsilon) \quad \text { and } \quad \rho_{3}=\sqrt{\epsilon} \frac{1}{\kappa \sqrt{\Psi_{3}(\kappa, \epsilon)}},
$$

where the existence and scaling of $\Psi_{3}$ follows from the weak approach of orbits to the steady state, with $\kappa$ chosen sufficiently small. (Here, we have also utilized the conservation of $r \hat{\epsilon}=\epsilon$ or, equivalently, of $\rho_{3}^{2} R_{3}=\epsilon$.) Finally, the continued manifold is tracked under the flow of (B.10) to the exit section

$$
\Sigma_{3}^{\text {in }}=\left\{\left(U_{3}, R_{3}, \rho_{3}\right) \mid \rho_{3}=\kappa\right\}
$$

in $\widehat{K}_{3}$, where we find the expansion

$$
U_{3}=-\frac{\gamma}{\alpha \sigma}-\sqrt{\epsilon} \Phi_{3}(\kappa, \epsilon) \quad \text { and } \quad R_{3}=\frac{\epsilon}{\kappa^{2}} .
$$

Reverting to $(\hat{u}, \hat{r}, \hat{\epsilon})$-coordinates, we obtain the desired expansion for the corresponding orbit within the section $\Sigma^{\text {in }}$, which concludes the proof of Lemma 6.10 .

\section{References}

[1] M. Beck, A. Doelman, and T. Kaper. A geometric construction of traveling waves in a bioremediation model. Journal of Nonlinear Science, 16(4):329-349, 2006.

[2] E. Brunet and B. Derrida. Shift in the velocity of a front due to a cutoff. Phys. Rev. E (3), 56(3, part A):2597-2604, 1997.

[3] J. Carr. Applications of centre manifold theory, volume 35 of Applied Mathematical Sciences. Springer-Verlag, New York, 1981.

[4] S.-N. Chow, C. Z. Li, and D. Wang. Normal forms and bifurcation of planar vector fields. Cambridge University Press, Cambridge, 1994.

[5] S.-N. Chow, W. Liu, and Y. Yi. Center manifolds for smooth invariant manifolds. Trans. Amer. Math. Soc., 352(11):5179-5211, 2000.

[6] W. Coppel. Dichotomies in stability theory. Lecture Notes in Mathematics, 629:1-98, 1978.

[7] P. De Maesschalck. Ackerberg-O'Malley resonance in boundary value problems with a turning point of any order. Commun. Pure Appl. Anal., 6(2):311-333, 2007. 
[8] F. Dumortier. Techniques in the theory of local bifurcations: blow-up, normal forms, nilpotent bifurcations, singular perturbations. In Bifurcations and periodic orbits of vector fields (Montreal, PQ, 1992), volume 408 of NATO Adv. Sci. Inst. Ser. C Math. Phys. Sci., pages 19-73. Kluwer Acad. Publ., Dordrecht, 1993.

[9] F. Dumortier, N. Popović, and T. J. Kaper. The critical wave speed for the Fisher-KolmogorovPetrowskii-Piscounov equation with cut-off. Nonlinearity, 20(4):855, 2007.

[10] F. Dumortier and R. H. Roussarie. Canard cycles and center manifolds, volume 577. American Mathematical Soc., 1996.

[11] N. Fenichel. Geometric singular perturbation theory for ordinary differential equations. $J$. Differential Equations, 31(1):53-98, 1979.

[12] A. Ghazaryan and V. Manukian. Coherent structures in a population model for mussel-algae interaction. SIAM Journal on Applied Dynamical Systems, 14(2):893-913, 2015.

[13] I. Gucwa and P. Szmolyan. Geometric singular perturbation analysis of an autocatalator model. Discrete Contin. Dyn. Syst. Ser. S, 2(4):783-806, 2009.

[14] A. J. Homburg and B. Sandstede. Chapter 8 - Homoclinic and Heteroclinic Bifurcations in Vector Fields. In F. T. Henk Broer and B. Hasselblatt, editors, Handbook of Dynamical Systems, volume 3 of Handbook of Dynamical Systems, pages 379 - 524. Elsevier Science, 2010.

[15] C. K. R. T. Jones. Geometric singular perturbation theory. In Dynamical systems (Montecatini Terme, 1994), volume 1609 of Lecture Notes in Math., pages 44-118. Springer, Berlin, 1995.

[16] C. K. R. T. Jones, T. J. Kaper, and N. Kopell. Tracking invariant manifolds up to exponentially small errors. SIAM Journal on Mathematical Analysis, 27(2):558-577, 1996.

[17] C. K. R. T. Jones and N. Kopell. Tracking invariant manifolds with differential forms in singularly perturbed systems. J. Differential Equations, 108(1):64-88, 1994.

[18] C. K. R. T. Jones and S.-K. Tin. Generalized exchange lemmas and orbits heteroclinic to invariant manifolds. Discrete Contin. Dyn. Syst. Ser. S, 2(4):967-1023, 2009.

[19] C. A. Klausmeier. Regular and irregular patterns in semiarid vegetation. Science, 284(5421):1826-1828, 1999.

[20] I. Kosiuk and P. Szmolyan. Geometric analysis of the Goldbeter minimal model for the embryonic cell cycle. Journal of Mathematical Biology, pages 1-32, 2015.

[21] M. Krupa and P. Szmolyan. Extending geometric singular perturbation theory to nonhyperbolic points - fold and canard points in two dimensions. SIAM Journal on Mathematical Analysis, 33(2):286-314, 2001.

[22] C. Kuehn. Normal hyperbolicity and unbounded critical manifolds. Nonlinearity, 27(6):13511366, 2014.

[23] C. Kuehn and P. Szmolyan. Multiscale geometry of the Olsen model and non-classical relaxation oscillations. Journal of Nonlinear Science, 25(3):583-629, 2015.

[24] P. A. Lagerstrom. Matched asymptotic expansions. Ideas and techniques, volume 76 of Applied Mathematical Sciences. Springer-Verlag, New York, 1988. 
[25] Q.-X. Liu, A. Doelman, V. Rottschäfer, M. de Jager, P. M. J. Herman, M. Rietkerk, and J. van de Koppel. Phase separation explains a new class of self-organized spatial patterns in ecological systems. Proc. Natl. Acad. Sci. USA, 110(29):11905-11910, 2013.

[26] W. Liu. Exchange lemmas for singular perturbation problems with certain turning points. Journal of Differential Equations, 167(1):134 - 180, 2000.

[27] A. B. Rovinsky and M. Menzinger. Chemical instability induced by a differential flow. Phys. Rev. Lett., 69:1193-1196, Aug 1992.

[28] R. A. Satnoianu and M. Menzinger. Non-Turing stationary patterns in flow-distributed oscillators with general diffusion and flow rates. Phys. Rev. E, 62:113-119, Jul 2000.

[29] R. A. Satnoianu, J. H. Merkin, and S. K. Scott. Spatio-temporal structures in a differential flow reactor with cubic autocatalator kinetics. Physica D: Nonlinear Phenomena, 124(4):345 - 367, 1998.

[30] S. Schecter. Exchange lemmas 2: General exchange lemma. J. Differential Equations, 245(2):411 - 441, 2008.

[31] S. Schecter. Exchange lemmas. I. Deng's lemma. J. Differential Equations, 245(2):392-410, 2008.

[32] J. A. Sherratt. Pattern solutions of the Klausmeier model for banded vegetation in semi-arid environments i. Nonlinearity, 23(10):2657, 2010.

[33] J. A. Sherratt. Pattern solutions of the Klausmeier model for banded vegetation in semiarid environments iv: Slowly moving patterns and their stability. SIAM Journal on Applied Mathematics, 73(1):330-350, 2013.

[34] E. Siero, A. Doelman, M. B. Eppinga, J. D. M. Rademacher, M. Rietkerk, and K. Siteur. Striped pattern selection by advective reaction-diffusion systems: Resilience of banded vegetation on slopes. Chaos: An Interdisciplinary Journal of Nonlinear Science, 25(3):-, 2015.

[35] C. Soto-Treviño. A geometric method for periodic orbits in singularly-perturbed systems. In Multiple-time-scale dynamical systems (Minneapolis, MN, 1997), volume 122 of IMA Vol. Math. Appl., pages 141-202. Springer, New York, 2001.

[36] J. van de Koppel, M. Rietkerk, N. Dankers, and P. M. J. Herman. Scale-dependent feedback and regular spatial patterns in young mussel beds. The American Naturalist, 165(3):pp. E66E77, 2005.

[37] W. van Saarloos. Front propagation into unstable states. Physics Reports, 386(2-6):29 - 222, 2003.

[38] A. Vanderbauwhede. Centre manifolds, normal forms and elementary bifurcations. In Dynamics reported, Vol. 2, volume 2 of Dynam. Report. Ser. Dynam. Systems Appl., pages 89-169. Wiley, Chichester, 1989.

[39] R.-H. Wang, Q.-X. Liu, G.-Q. Sun, Z. Jin, and J. van de Koppel. Nonlinear dynamic and pattern bifurcations in a model for spatial patterns in young mussel beds. Journal of The Royal Society Interface, 6(37):705-718, 2009. 
[40] M. Wechselberger. Extending Melnikov theory to invariant manifolds on non-compact domains. Dynamical Systems, 17(3):215-233, 2002. 